\title{
Hydrides: Solid State Transition Metal Complexes
}

\section{Klaus Yvon \& Guillaume Renaudin}

Volume III, pp. 1814-1846

in

\author{
Encyclopedia of Inorganic Chemistry, Second Edition \\ (ISBN 0-470-86078-2) \\ Editor-in-Chief: \\ R. Bruce King
}

(C) John Wiley \& Sons, Ltd, Chichester, 2005 


\section{Hydrides: Solid State Transition Metal Complexes}

\author{
Klaus Yvon \& Guillaume Renaudin \\ University of Geneva, Geneva, Switzerland
}

1 Introduction

2 Experimental Details

3 Hydride Structure Types and Properties

4 Crystal Chemistry

5 Properties

6 Conclusions

7 Related Articles

8 References prismatic; $Z=$ Number of formula units per crystallographic unit cell.

\section{Glossary}

Complex metal hydride: solid-state compound containing homoleptic, anionic metal-hydrogen complexes

$d^{1}, d^{2}, d^{3}$ etc: electron configuration of transition element

$\Delta H$ : enthalpy of hydride formation as determined from pressure-composition isotherms during desorption by using van't Hoff's method

Homoleptic hydride complexes: complexes having hydrogen ligands only

Hydrido complexes: structural units formed by central metal atoms and terminal hydrogen ligands

\section{Abbreviations}

$\mathrm{APW}=$ Augmented plane wave $\mathrm{HT}=$ High temperature; INS = Inelastic neutron scattering, IR = Infrared; lif = Limiting ionic formula; lin = Linear; LCAO-MO = Linear combination of atomic orbitals-molecular orbitals; LT = Low temperature; $\mathrm{M}=$ Alkali ( $\mathrm{Li}, \mathrm{Na}, \mathrm{K}, \mathrm{Rb}, \mathrm{Cs}$ ), alkaline earth $(\mathrm{Mg}, \mathrm{Ca}, \mathrm{Sr}, \mathrm{Ba})$, divalent $(\mathrm{Yb}, \mathrm{Eu})$ or trivalent (La, Nd) lanthanide; NMR = Nuclear magnetic resonance; $\mu_{\text {eff }}=$ Magnetic moment in units of Bohr magneton, $\mu_{\mathrm{B}}$; $\mathrm{npd}=$ Neutron powder diffraction; oct $=$ Octahedral; $\mathrm{pbp}=$ Pentagonal bipyramidal; sad = Saddle-like; spl = Square planar; spy = Square pyramidal; SQUID = Superconducting quantum interference device; $\mathrm{T}$-metal $=$ Transition metal (Mn, Fe, Co, Ni, Cu, Zn, Tc, Ru, Rh, Pd, Cd, Re, Os, Ir, Pt); $T_{\mathrm{C}}=$ Curie temperature; dis $=$ Disordered; tet $=$ Tetrahedral; tri $=$ Triangular; $\quad$ s $=\mathrm{T}$-shaped; $\mathrm{ttp}=$ Tricapped trigonal

\section{INTRODUCTION}

This article is an update of a review written ${ }^{\mathbf{1}}$ some 10 years ago. It covers the currently known solid-state complex homoleptic transition (T) metal hydrido complexes and some of their properties of interest for science and technology. The complexes occur in a wide variety of solid-state compounds. Historically, the first fully characterized example is $\mathrm{K}_{2} \mathrm{ReH}_{9}$. Its structure was reported in 1964 by Abrahams, Ginsberg, and $\mathrm{Knox}^{2}{ }^{2}$ and found to contain tricapped trigonal prismatic $\left[\mathrm{ReH}_{9}\right]^{2-}$ complex anions. The second member is $\mathrm{Sr}_{2} \mathrm{RuH}_{6}$, which was reported in the 1970 s by Moyer and collaborators, ${ }^{3}$ was found to contain octahedral $\left[\mathrm{RuH}_{6}\right]^{4-}$ complexes. Wider interest in this type of hydride started only in the 1980s after the discovery of ternary transition metal hydrides such as $\mathrm{LaNi}_{5} \mathrm{H}_{6}$ and $\mathrm{FeTiH}_{2}$, which were suitable media for reversible hydrogen storage (for useful reviews see books and journal issues edited by Schlapbach). ${ }^{4}$ The compounds showed metallic properties and had a large homogeneity range with respect to hydrogen. One of them, however, $\mathrm{Mg}_{2} \mathrm{NiH}_{4}$, was nonmetallic and had a fixed hydrogen content. Originally reported in 1968 by Reilly and Wiswall, ${ }^{5}$ it was classified as a hydride containing a T-metal hydrido complex only 18 years later, after its structure was fully characterized by Zolliker et al. ${ }^{6}$ and found to contain tetrahedral $\left[\mathrm{NiH}_{4}\right]^{4-}$ units called 'complexes'. In the meantime, other homoleptic hydrido complexes were discovered, such as square-pyramidal $\left[\mathrm{CoH}_{5}\right]^{4-}$, square-planar $\left[\mathrm{PtH}_{4}\right]^{2-}$, and linear $\left[\mathrm{PdH}_{2}\right]^{2-}$, and also hydrides that contained both complex bonded hydrogen and ionic hydrogen not bonded to the transition metal. In 1991, some 13 ternary hydride structure types were known. Some of them were reviewed and named 'complex transition metal hydrides' by Bronger, ${ }^{7}$ a term now widely accepted. The first comprehensive review as written in 1993 by Yvon ${ }^{1}$ covered 25 different transition metal hydride complexes in some 69 compounds, including quaternary hydrides. Over the years, the number of complex metal hydrides increased continuously and reviews appeared on various of their aspects such as synthetics, ${ }^{8,9}$ diffraction methodology, ${ }^{10,11}$ bonding, ${ }^{12-14}$ crystal chemistry, ${ }^{\mathbf{1 5 , 1 6}}$ complex formation in hydrogenated intermetallic compounds, ${ }^{\mathbf{1 7}}$ and materials science. ${ }^{\mathbf{1 8 , 1 9}}$ The hydrides were also incorporated into a public online database. ${ }^{20}$ At present, over 127 complex T-metal hydrides covering 47 structure types are known, that is, since the 1993 review their number has almost doubled. In this update, both mononuclear (terminal hydrogen ligands only) and polynuclear (terminal and bridging hydrogen ligands) 
complexes are covered. Only those hydrides are treated whose structures are fully characterized. Most contain well-defined T-metal hydride complexes and show nonmetallic behavior. Some, however, do not, and their classification as 'complex' hydrides is debatable. They are, nevertheless, included here because they illustrate the continuous transition that exists between complex and interstitial (metallic) hydrides. Among the aspects discussed are factors that govern the formation of complex hydrides, hydrogen contents, and thermal stability. These factors are not only of fundamental interest but also of practical relevance, because they determine the potential of metal hydrides for hydrogen storage applications.

\section{EXPERIMENTAL DETAILS}

\subsection{Compositions and Structures}

The presently known complex transition metal hydrides are based on late $3 \mathrm{~d}, 4 \mathrm{~d}$, and $5 \mathrm{~d}$ transition elements of groups 7-10, and on monovalent, divalent, and trivalent metals (M) belonging to the alkali, alkaline earth, and/or lanthanide series, respectively. For completeness, complexes based on closed-shell elements of group $11(\mathrm{Cu}$, etc.) and 12 (Zn, etc.) are also included. Most hydrides are true ternary (or quaternary) compounds in the sense that they do not derive from stable intermetallic compounds, but form only in the presence of hydrogen. Some hydrides, however, do and show interesting physical phenomena. The metal ratios are usually situated in the range $\mathrm{M} / \mathrm{T}=1-4$, and the hydrogen contents in the range $\mathrm{H} /(\mathrm{M}+\mathrm{T})=1-4.5$. The compounds crystallize with 47 different, mostly new, structure types and represent over 127 hydrides. The first representative of each hydride structure type and the isostructural compounds known are listed in chronological order in Table 1 (year of publication in parentheses).

There exist two broad families of complex transition metal hydrides. The first contains hydrogen bonded to transition elements only and has the general composition

$$
\mathrm{M}_{m}^{\delta+}\left[\mathrm{TH}_{n}\right]^{\delta-}(m, n, \delta=1,2,3 \ldots)
$$

where $\left[\mathrm{TH}_{n}\right]^{\delta-}$ are complexes that are stabilized by charge transfer from the surrounding metal cations $\mathrm{M}^{\delta+}$. The second family contains hydrogen bonded to $\mathrm{T}$ elements and hydride anions $\mathrm{H}^{-}$bonded to metal cations $\mathrm{M}^{\delta+}$ only, corresponding to the general composition

$$
\mathrm{M}_{m}{ }^{\delta+}\left[\mathrm{TH}_{n}\right]^{\delta-} \mathrm{M}_{\mathrm{o}}{ }^{\delta+} \mathrm{H}^{-} p(m, n, o, p, \delta=1,2,3 \ldots)
$$

These 'composite' hydrides are of particular interest because they combine different types of metal-hydrogen bonding in the same structure.
Table 1 Complex transition metal hydride structure types and

\begin{tabular}{|c|c|c|}
\hline $\begin{array}{l}\text { Structure } \\
\text { types }\end{array}$ & Representatives & $\begin{array}{l}\text { Complex transition } \\
\text { metal hydrides }\end{array}$ \\
\hline I & $\mathrm{K}_{2} \mathrm{ReH}_{9}(1964)$ & $\mathrm{K}_{2} \mathrm{TH}_{9}(\mathrm{~T}=\mathrm{Tc}, \mathrm{Re})$ \\
\hline II & $\mathrm{Sr}_{2} \mathrm{RuH}_{6}(1971)$ & $\begin{array}{l}\mathrm{M}_{2} \mathrm{FeH}_{6}(\mathrm{M}=\mathrm{Mg}, \mathrm{Ca}, \mathrm{Sr}, \mathrm{Eu}, \\
\mathrm{Yb}) \\
\mathrm{M}_{2} \mathrm{RuH}_{6}(\mathrm{M}=\mathrm{Mg}, \mathrm{Ca}, \mathrm{Sr}, \mathrm{Ba}, \\
\mathrm{Eu}, \mathrm{Yb}) \\
\mathrm{M}_{2} \mathrm{OsH}_{6}(\mathrm{M}=\mathrm{Mg}, \mathrm{Ca}, \mathrm{Sr}, \mathrm{Ba}) \\
\mathrm{M}_{2} \mathrm{PtH}_{6}(\mathrm{M}=\mathrm{Na}, \mathrm{K}, \mathrm{Rb}, \mathrm{Cs})\end{array}$ \\
\hline III & $\mathrm{Sr}_{2} \mathrm{IrH}_{5}(1971)$ & $\begin{array}{l}\mathrm{Mg}_{2} \mathrm{CoH}_{5} \\
\mathrm{M}_{2} \mathrm{RhH}_{5+x}(\mathrm{M}=\mathrm{Ca}, \mathrm{Sr}, \mathrm{Eu}) \\
\mathrm{M}_{2} \operatorname{IrH}_{5+x}(\mathrm{M}=\mathrm{Mg}, \mathrm{Ca}, \mathrm{Sr}, \mathrm{Eu})\end{array}$ \\
\hline IV & $\mathrm{Na}_{2} \mathrm{PtH}_{4}(1984)$ & $\begin{array}{l}\mathrm{M}_{2} \mathrm{PdH}_{4}(\mathrm{M}=\mathrm{Na}, \mathrm{K}) \\
\mathrm{Na}_{2} \mathrm{PtH}_{4}\end{array}$ \\
\hline $\mathrm{V}$ & $\mathrm{Mg}_{2} \mathrm{NiH}_{4}(1986)$ & $\mathrm{Mg}_{2} \mathrm{NiH}_{4}$ \\
\hline VI & $\mathrm{K}_{2} \mathrm{PtH}_{4}(1986)$ & $\begin{array}{l}\mathrm{M}_{2} \mathrm{PdH}_{4}(\mathrm{M}=\mathrm{Rb}, \mathrm{Cs}) \\
\mathrm{M}_{2} \mathrm{PtH}_{4}(\mathrm{M}=\mathrm{K}, \mathrm{Rb}, \mathrm{Cs})\end{array}$ \\
\hline VII & $\mathrm{Na}_{2} \mathrm{PdH}_{2}(1988)$ & $\mathrm{M}_{2} \mathrm{PdH}_{2}(\mathrm{M}=\mathrm{Li}, \mathrm{Na})$ \\
\hline VIII & $\mathrm{K}_{3} \mathrm{PtH}_{5}(1988)$ & $\begin{array}{l}\mathrm{M}_{3} \mathrm{TH}_{5}(\mathrm{M}=\mathrm{K}, \mathrm{Rb}, \mathrm{Cs} \text { and } \\
\mathrm{T}=\mathrm{Pd}, \mathrm{Pt})\end{array}$ \\
\hline IX & $\mathrm{K}_{3} \mathrm{PdH}_{3}(1990)$ & $\mathrm{M}_{3} \mathrm{PdH}_{3}(\mathrm{M}=\mathrm{K}, \mathrm{Rb}, \mathrm{Cs})$ \\
\hline $\mathrm{X}$ & $\mathrm{CaPdH}_{2}(1990)$ & $\begin{array}{l}\mathrm{MPdH}_{3-x}(\mathrm{M}=\mathrm{Ca}, \mathrm{Sr}, \mathrm{Eu}, \mathrm{Yb}) \\
\mathrm{MNidH}_{3-x}(\mathrm{M}=\mathrm{Ca}, \mathrm{Yb})\end{array}$ \\
\hline XI & $\mathrm{Li}_{4} \mathrm{RuH}_{6}(1991)$ & $\begin{array}{l}\mathrm{M}_{4} \mathrm{RuH}_{6}(\mathrm{M}=\mathrm{Li}, \mathrm{Na}) \\
\mathrm{Li}_{4} \mathrm{OsH}_{6}\end{array}$ \\
\hline XII & $\mathrm{Na}_{3} \mathrm{RhH}_{6}(1991)$ & $\begin{array}{l}\mathrm{M}_{3} \mathrm{TH}_{6}(\mathrm{M}=\mathrm{Li}, \mathrm{Na} \text { and } \\
\mathrm{T}=\mathrm{Rh}, \mathrm{Ir})\end{array}$ \\
\hline XIII & $\mathrm{Li}_{3} \mathrm{RhH}_{4}(1991)$ & $\mathrm{Li}_{3} \mathrm{RhH}_{4}$ \\
\hline XIV & $\mathrm{MgRhH}_{1-x}(1992)$ & $\mathrm{MgRhH}_{0.94}$ \\
\hline $\mathrm{XV}$ & $\mathrm{Mg}_{3} \mathrm{RuH}_{3}(1992)$ & $\mathrm{Mg}_{3} \mathrm{RuH}_{3}$ \\
\hline XVI & $\mathrm{SrMg}_{2} \mathrm{FeH}_{8}(1992)$ & $\mathrm{MMg}_{2} \mathrm{FeH}_{8}(\mathrm{M}=\mathrm{Sr}, \mathrm{Ba}, \mathrm{Eu})$ \\
\hline XVII & $\mathrm{Mg}_{6} \mathrm{Co}_{2} \mathrm{H}_{11}(1992)$ & $\mathrm{Mg}_{6} \mathrm{Co}_{2} \mathrm{H}_{11}$ \\
\hline XVIII & $\mathrm{Mg}_{2} \mathrm{RuH}_{4}(1992)$ & $\mathrm{Mg}_{2} \mathrm{RuH}_{4}$ \\
\hline XIX & $\mathrm{CaMgNiH}_{4}(1992)$ & $\begin{array}{l}\mathrm{MMgNiH}_{4}(\mathrm{M}=\mathrm{Ca}, \mathrm{Sr}, \mathrm{Eu}, \\
\mathrm{Yb})\end{array}$ \\
\hline $\mathrm{XX}$ & $\begin{array}{l}\mathrm{Ca}_{4} \mathrm{Mg}_{4} \mathrm{Fe}_{3} \mathrm{H}_{22} \\
\quad(1992)\end{array}$ & $\mathrm{M}_{4} \mathrm{Mg}_{4} \mathrm{Fe}_{3} \mathrm{H}_{22}(\mathrm{M}=\mathrm{Ca}, \mathrm{Yb})$ \\
\hline XXI & $\mathrm{Ba}_{2} \mathrm{PtH}_{6}(1993)$ & $\mathrm{M}_{2} \mathrm{PtH}_{6}(\mathrm{M}=\mathrm{Sr}, \mathrm{Ba})$ \\
\hline XXII & $\mathrm{LiSr}_{2} \mathrm{PdH}_{5}(1993)$ & $\mathrm{LiSr}_{2} \mathrm{PdH}_{5}$ \\
\hline XXIII & $\mathrm{Mg}_{3} \mathrm{ReH}_{7}(1993)$ & $\mathrm{Mg}_{3} \mathrm{TH}_{7}(\mathrm{~T}=\mathrm{Mn}, \mathrm{Re})$ \\
\hline XXIV & $\mathrm{Mg}_{4} \mathrm{IrH}_{5}(1993)$ & $\mathrm{Mg}_{4} \mathrm{IrH}_{5}$ \\
\hline XXV & $\mathrm{Mg}_{3} \mathrm{RuH}_{6}(1993)$ & $\mathrm{Mg}_{3} \mathrm{RuH}_{6}$ \\
\hline XXVI & $\mathrm{Sr}_{8} \mathrm{Rh}_{5} \mathrm{H}_{23}(1994)$ & $\mathrm{M}_{8} \mathrm{Rh}_{5} \mathrm{H}_{23}(\mathrm{M}=\mathrm{Ca}, \mathrm{Sr})$ \\
\hline XXVII & $\mathrm{LiMg}_{2} \mathrm{RuH}_{7}(1994)$ & $\mathrm{LiMg}_{2} \mathrm{TH}_{7}(\mathrm{~T}=\mathrm{Ru}, \mathrm{Os})$ \\
\hline XXVIII & $\mathrm{BaReH}_{9}(1994)$ & $\mathrm{BaReH}_{9}$ \\
\hline XXIX & $\mathrm{K}_{2} \mathrm{ZnH}_{4}(1994)$ & $\begin{array}{l}\mathrm{M}_{2} \mathrm{ZnH}_{4}(\mathrm{M}=\mathrm{K}, \mathrm{Rb}, \mathrm{Cs}) \\
\mathrm{M}_{2} \mathrm{PdH}_{4}(\mathrm{M}=\mathrm{Sr}, \mathrm{Ba}, \mathrm{Eu})\end{array}$ \\
\hline XXX & $\mathrm{K}_{3} \mathrm{ZnH}_{5}(1994)$ & $\begin{array}{l}\mathrm{M}_{3} \mathrm{TH}_{5}(\mathrm{M}=\mathrm{K}, \mathrm{Rb}, \mathrm{Cs} \text { and } \\
\mathrm{T}=\mathrm{Mn}, \mathrm{Zn}) \\
\mathrm{Cs}_{3} \mathrm{CdH}_{5}\end{array}$ \\
\hline XXXI & $\mathrm{Ba}_{3} \mathrm{Ir}_{2} \mathrm{H}_{12}(1994)$ & $\mathrm{Ba}_{3} \mathrm{Ir}_{2} \mathrm{H}_{12}$ \\
\hline XXXII & $\begin{array}{l}\mathrm{Ca}_{4} \mathrm{Mg}_{4} \mathrm{Co}_{3} \mathrm{H}_{19} \\
\quad(1995)\end{array}$ & $\mathrm{M}_{4} \mathrm{Mg}_{4} \mathrm{Co}_{3} \mathrm{H}_{19}(\mathrm{M}=\mathrm{Ca}, \mathrm{Yb})$ \\
\hline XXXIII & $\mathrm{KNaReH}_{9}(1995)$ & $\mathrm{KNaReH}_{9}$ \\
\hline XXXIV & $\mathrm{Li}_{5} \mathrm{Pt}_{2} \mathrm{H}_{9}(1995)$ & $\mathrm{Li}_{5} \mathrm{Pt}_{2} \mathrm{H}_{9}$ \\
\hline XXXV & $\mathrm{Ba}_{7} \mathrm{Cu}_{3} \mathrm{H}_{17}(1996)$ & $\mathrm{Ba}_{7} \mathrm{Cu}_{3} \mathrm{H}_{17}$ \\
\hline XXXVI & $\begin{array}{l}\mathrm{LiMg}_{4} \mathrm{Os}_{2} \mathrm{H}_{13} \\
\quad(1996)\end{array}$ & $\mathrm{LiMg}_{4} \mathrm{~T}_{2} \mathrm{H}_{13}(\mathrm{~T}=\mathrm{Ru}, \mathrm{Os})$ \\
\hline XXXVII & $\mathrm{Li}_{2} \mathrm{PtH}_{2}(1996)$ & $\mathrm{Li}_{2} \mathrm{PtH}_{2}$ \\
\hline XXXVIII & $\begin{array}{l}\mathrm{BaMg}_{2} \mathrm{RuH}_{8} \\
\quad(1997)\end{array}$ & $\mathrm{BaMg}_{2} \mathrm{TH}_{8}(\mathrm{~T}=\mathrm{Ru}, \mathrm{Os})$ \\
\hline XXXIX & $\mathrm{NaBaPdH}_{3}(1998)$ & $\mathrm{NaBaPdH}_{3}$ \\
\hline
\end{tabular}
representatives 
Table 1 cont'd

\begin{tabular}{lll}
\hline $\begin{array}{l}\text { Structure } \\
\text { types }\end{array}$ & Representatives & $\begin{array}{l}\text { Complex transition } \\
\text { metal hydrides }\end{array}$ \\
\hline XL & $\mathrm{K}_{3} \mathrm{ReH}_{6}(1998)$ & $\mathrm{K}_{3} \mathrm{ReH}_{6}$ \\
XLI & $\mathrm{Ca}_{8} \mathrm{Rh}_{6} \mathrm{H}_{24}(1998)$ & $\mathrm{Ca}_{8} \mathrm{Rh}_{6} \mathrm{H}_{24}$ \\
XLII & $\mathrm{Rb}_{3} \mathrm{ReH}_{10}(1998)$ & $\mathrm{M}_{3} \mathrm{ReH}_{10}(\mathrm{M}=\mathrm{K}, \mathrm{Rb}, \mathrm{Cs})$ \\
XLIII & $\mathrm{Na}_{3} \mathrm{OsH}_{7}(2002)$ & $\mathrm{Na}_{3} \mathrm{TH}_{7}(\mathrm{~T}=\mathrm{Ru}, \mathrm{Os})$ \\
XLIV & $\mathrm{Cs}_{3} \mathrm{OsH}_{9}(2002)$ & $\mathrm{M}_{3} \mathrm{OsH}_{9}(\mathrm{M}=\mathrm{Rb}, \mathrm{Cs})$ \\
XLV & $\mathrm{Mg}_{6} \mathrm{Ir}_{2} \mathrm{H}_{11}(2002)$ & $\mathrm{Mg}_{6} \mathrm{Ir}_{2} \mathrm{H}_{11}$ \\
XLVI & $\mathrm{NdMgNi}_{4} \mathrm{H}_{4}$ & $\mathrm{MMgNi}_{4} \mathrm{H}_{\sim 4}(\mathrm{M}=\mathrm{La}, \mathrm{Nd})$ \\
& $(2003)$ & \\
XLVII & $\mathrm{LaMg}_{2} \mathrm{NiH}_{7}(2003)$ & $\mathrm{LaMg}_{2} \mathrm{NiH}_{7}$ \\
\hline
\end{tabular}

\subsection{Synthesis}

The most common route of synthesis is by solid-state reaction, such as sintering powder mixtures of the elements, binary alloys, and/or binary metal hydrides at relatively high pressures (up to $160 \mathrm{bar}$ ) and moderate temperatures $(<1000 \mathrm{~K})$. Synthesis from binary metal compounds is rarely possible because such compounds either do not exist, such as in the $\mathrm{Mg}-\mathrm{Fe}$ and $\mathrm{Mg}-\mathrm{Os}$ systems, or form at metal ratios that differ significantly from those of the ternary (or quaternary) complex metal hydrides, such as $\mathrm{Mg}_{2} \mathrm{CoH}_{5}$ $\left(\mathrm{MgCo}, \mathrm{MgCo}_{2}\right)$ or $\mathrm{Mg}_{2} \mathrm{RuH}_{6}\left(\mathrm{Mg}_{3} \mathrm{Ru}_{2}\right)$. Some hydrides derive from two-phase mixtures such as $\mathrm{Ba}_{7} \mathrm{Cu}_{3} \mathrm{H}_{17}(\mathrm{Ba}-\mathrm{Cu}$ alloy with compositional ratio $\mathrm{Ba} / \mathrm{Cu} \sim 7 / 3$ ) while others can be prepared by inexpensive ball milling $\left(\mathrm{Mg}_{2} \mathrm{FeH}_{6}\right)$ and combustion synthesis $\left(\mathrm{Mg}_{2} \mathrm{NiH}_{4}\right)$. Solution methods, such as those employed to synthesize $\mathrm{K}_{2} \mathrm{ReH}_{9}$ and related hydrides, are not yet fully explored. The only other soluble Tmetal hydride complex known is $\left[\mathrm{FeH}_{6}\right]^{4-}{ }^{21}$ The solid-state reaction products are mostly polycrystalline and often colored. They usually contain impurity phases (nonreacted $\mathrm{T}$ and/or binary $\mathrm{M}$ metal hydrides), but rarely contain single crystals of sufficient size and quality for diffraction measurements or measurements of physical properties. Those based on alkali and heavy alkaline earth metals are often sensitive to air and moisture.

A decisive parameter for hydride formation is pressure. The synthesis of hydrides based on light transition elements usually requires higher hydrogen pressures than those based on heavier congeners. Examples are the palladium compounds $\mathrm{M}_{2} \mathrm{PdH}_{4}$ and $\mathrm{M}_{3} \mathrm{PdH}_{5}$, which form at 20 and 70 bar, respectively, in contrast to their platinum congeners, that form already at 1 bar. For a given transition metal, an increase of hydrogen pressure usually allows the stabilization of higher formal oxidation states. Examples are found for palladium in $\mathrm{M}_{3} \mathrm{Pd}^{0} \mathrm{H}_{3}$ and $\mathrm{M}_{2} \mathrm{Pd}^{\mathrm{II}} \mathrm{H}_{4}$, which form at 1 and $20 \mathrm{bar}$, respectively, for platinum in $\mathrm{K}_{2} \mathrm{Pt}^{\mathrm{II}} \mathrm{H}_{4}$ and $\mathrm{K}_{2} \mathrm{Pt}^{\mathrm{IV}} \mathrm{H}_{6}$, which form at 1 and 1800 bar respectively, and for ruthenium in $\mathrm{Mg}_{2} \mathrm{Ru}^{0} \mathrm{H}_{4}$ and $\mathrm{Mg}_{2} \mathrm{Ru}^{\mathrm{II}} \mathrm{H}_{6}$, which form at 20 and 90 bar, respectively. However, the inverse situation prevails for manganese based $\mathrm{Mg}_{3} \mathrm{Mn}^{\mathrm{I}} \mathrm{H}_{7}$, which forms under a mechanical pressure of
$20 \mathrm{kbar}$, while $\mathrm{K}_{3} \mathrm{Mn}^{\mathrm{II}} \mathrm{H}_{5}$ forms under a hydrogen gas pressure of only $3 \mathrm{kbar}$.

\subsection{Structure Analysis}

Owing to the low X-ray scattering power of hydrogen, neutron diffraction experiments on deuterides are necessary. Most atomic arrangements are determined on powders, and this may cause difficulties owing to poor crystallinity, the presence of impurity phases (often not detected by $\mathrm{X}$ rays), structural complexity $\left(\mathrm{Mg}_{6} \mathrm{Ir}_{2} \mathrm{D}_{11}\right.$, e.g., has 126 free positional parameters), heavy absorption (Eu, Cd), microtwinning owing to temperature induced phase transitions, and disorder in the hydrogen complex ligand spheres. High-resolution measurements (e.g. with synchrotron radiation) are rare. The data are usually analyzed by the Rietveld method. For better convergence, the number of refined parameters, in particular, those of the atomic displacement amplitudes, are reduced by constraints. For all these reasons, hydride structures are generally less well characterized than other solid-state structures.

\subsection{Properties}

Most compounds are nonmetallic. Only few other properties are known. The main reason for this is the lack of single crystals, the presence of impurity phases in the samples, and the sensitivity of many compounds to air and moisture. Data on magnetic, electric, and spectroscopic (NMR, Mössbauer, IR) properties are scarce. Enthalpies of hydride formation as measured from pressure-composition isotherms are known for hydrides based on $3 \mathrm{~d}$ elements only. Theoretical band structure calculations are available only on relatively simple crystal structures. Structural dynamics are investigated by spectroscopic methods (INS, Raman, IR, NMR) and magnetic properties on SQUID magnetometers. Owing to the absence of single crystals of suitable size, reliable measurements of the electric conductivity are rare. Enthalpies of hydride formation, $\Delta H$, are usually determined from pressure-composition isotherms as measured on Sievert's apparatus or on a thermobalance, by using the relation

$$
\ln \left(p_{\text {eq }}\right)=\frac{-\Delta H}{R \cdot T}+\frac{\Delta S}{R} \quad \text { Van't Hoff equation }
$$

where $p_{\text {eq }}$ is the hydrogen equilibrium pressure and $\mathrm{T}$ the absolute temperature. Given that the entropy term, $\Delta S$, does not much change from one system to another $\left(\Delta S \sim 130 \mathrm{~J} \mathrm{~K}^{-1} \mathrm{~mol}^{-1} \mathrm{H}_{2}\right.$ ) the hydrogen decomposition temperatures at a given pressure scale roughly with enthalpy ( $293 \mathrm{~K}: \Delta H \sim 30 \mathrm{~kJ} / \mathrm{H}_{2}$ for $p_{\text {eq }}=1$ bar). Finally, the reaction products ought to be handled with care because many are air sensitive and some are extremely pyrophoric. For these reasons, quite a few complex metal hydrides are known to exist but have not yet been fully characterized. 


\section{HYDRIDE STRUCTURE TYPES AND PROPERTIES}

Complex transition metal hydrides combine structural features typical for coordination compounds and ionic solids. They display covalently bonded hydrogen such as in molecular transition metal complexes ${ }^{22}$ (see Hydride Complexes of the Transition Metals), and hydride anions $\left(\mathrm{H}^{-}\right)$bonded to electropositive metals $\left(\mathrm{M}^{+}, \mathrm{M}^{2+}, \mathrm{M}^{3+}\right)$ only, such as in saline hydrides. In metal-rich systems, hydrogen has interstitial character, as in typically metallic transition metal hydrides. $^{23}$ The structural and physical properties of the 47 known hydride structure types and of their representatives (stated in parentheses) are summarized below. Limiting ionic formulas (lif) and the corresponding formal oxidation states and electron configurations of the transition elements are indicated whenever possible. Such formulas do not provide an adequate description of the bonding but allow one to rationalize the $\mathrm{H}$ content (see below). For each structure, T-D bond distances and shortest $\mathrm{M}-\mathrm{D}$ (complex $\mathrm{D}), \mathrm{M}-\mathrm{D}^{-}$(anionic $\mathrm{D}^{-}$) and $\mathrm{D}-\mathrm{D}$ distances are stated. For all structure types, illustrations are given (see Figures $1-47 ; \mathrm{TH}_{\mathrm{n}}$ complexes are drawn by heavy lines, disordered ligands by double lines, $\mathrm{M}-\mathrm{M}$ contacts surrounding $\mathrm{TH}_{n}$ complexes by single lines, $\mathrm{M}$ atoms as large circles, and $\mathrm{H}^{-}$ions as small isolated circles).

\section{$3.1 \mathrm{~K}_{2} \operatorname{ReH}_{9}\left(\mathrm{~K}_{2} \mathrm{TcH}_{9}\right)$}

From reaction of $\mathrm{KReO}_{4}$ with $\mathrm{K}$ metal in ethylenediamine-water solution; ${ }^{2}$ colorless crystals, unstable in moist air; NMR and IR data, optical absorption spectra and LCAO-MO calculations; ordered structure (Figure 1) from single-crystal neutron diffraction on hydride and later on deuteride: ${ }^{24} P \overline{6} 2 m, Z=3$; two different Re sites, occurring at the ratio $\operatorname{Re} 1: \operatorname{Re} 2=1: 2$, both forming $\operatorname{ttp}-\left[\mathrm{ReH}_{9}\right]^{2-}$ 18-electron complexes with $\mathrm{T}$ site symmetries $\overline{6} 2 \mathrm{~m}$ and $\overline{6}$, respectively; surrounded by two types of $\mathrm{K}$ cations; four $\mathrm{H}$ sites; lif: $2 \mathrm{~K}^{+} \cdot\left[\mathrm{ReH}_{9}\right]^{2-}$; $\mathrm{Re}^{\mathrm{VII}}, \mathrm{d}^{0}$; no structure data on Tc compound.

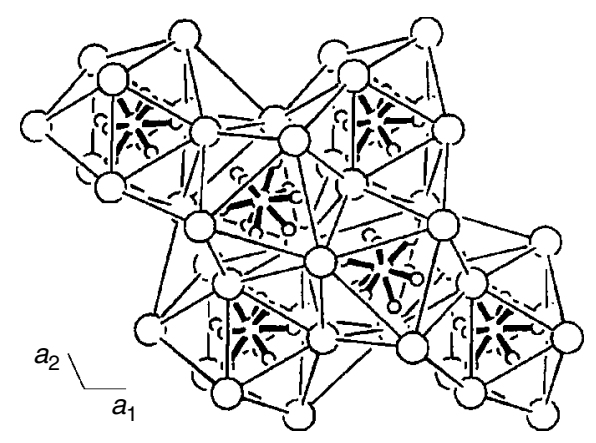

Figure 1 Structure of $\mathrm{K}_{2} \mathrm{ReH}_{9}$
Distances $(\AA):{ }^{24} \operatorname{Re}-H(3 \times)=1.68(\operatorname{Re} 1) ; 1.67(\operatorname{Re} 2)$; $\mathrm{Re}-\mathrm{H}(6 \times)=1.69(\operatorname{Re} 1) ; 1.70(\operatorname{Re} 2) ; \mathrm{K}-\mathrm{H}=2.69 ; \mathrm{H}-\mathrm{H}=$ 1.90 .

\section{2 $\mathrm{Sr}_{2} \mathrm{RuH}_{6}\left(\mathrm{M}_{2} \mathrm{FeH}_{6}, \mathrm{M}=\mathrm{Mg}, \mathrm{Ca}, \mathrm{Sr}, \mathrm{Yb}, \mathrm{Eu}\right.$; $\mathrm{M}_{2} \mathrm{RuH}_{6}, \mathrm{M}=$ any; $\mathrm{M}_{2} \mathrm{OsH}_{6}, \mathrm{M}=\mathrm{Mg}, \mathrm{Ca}, \mathrm{Sr}, \mathrm{Ba}$; $\left.\mathrm{M}_{2} \mathrm{PtH}_{6}, \mathrm{M}=\mathrm{Na}, \mathrm{K}, \mathrm{Rb}, \mathrm{Cs}\right)$}

$\mathrm{Sr}_{2} \mathrm{RuH}_{6}$ and isostructural hydrides $\mathrm{M}_{2} \mathrm{TH}_{6}$ from elemental $\mathrm{T}$ (Fe, $\mathrm{Ru}, \mathrm{Os}$ ) and binary hydrides $\mathrm{MH}_{2}$ or $\mathrm{Mg}$ by sintering at various temperatures and hydrogen pressures, ${ }^{\mathbf{3 , 2 5}-\mathbf{3 0}}$ or by mechanical alloying followed by sintering $(\mathrm{T}=\mathrm{Fe}) ;^{\mathbf{3 1}, 32}$ powders either colored $\left(\mathrm{Mg}_{2} \mathrm{FeH}_{6}\right.$, green; $\mathrm{Eu}_{2} \mathrm{RuH}_{6}$, red), or white-gray $\left(\mathrm{Ca}_{2} \mathrm{RuH}_{6}, \mathrm{Mg}_{2} \mathrm{OsH}_{6}\right)$, or black $\left(\mathrm{Yb}_{2} \mathrm{RuH}_{6}\right)$; moisture sensitive and partly pyrophoric $\left(\mathrm{Mg}_{2} \mathrm{OsH}_{6}\right) ; \mathrm{M}_{2} \mathrm{FeH}_{6}$ $(\mathrm{M}=\mathrm{Ca}, \mathrm{Sr}, \mathrm{Eu}), \mathrm{M}_{2} \mathrm{OsH}_{6}(\mathrm{M}=\mathrm{Mg}, \mathrm{Ca}), \mathrm{Mg}_{2} \mathrm{RuH}_{6}$ and various solid solutions; ${ }^{29} \mathrm{M}_{2} \mathrm{PtH}_{6}(\mathrm{M}=\mathrm{Na}, \mathrm{K}, \mathrm{Rb}, \mathrm{Cs})^{33-35}$ by reaction of alkaline hydride with platinum sponge at $770 \mathrm{~K}$ under high hydrogen pressure (between 2 and 4 kbar); $\mathrm{M}_{2} \mathrm{PtH}_{6}$ are colorless and very sensitive to moisture and air; IR stretching frequencies of $\mathrm{T}-\mathrm{H}(\mathrm{T}=\mathrm{Fe}, \mathrm{Ru}, \mathrm{Os})$ bonds range between 1450 and $1850 \mathrm{~cm}^{-1}$, and increase in the sequence $\mathrm{Fe}, \mathrm{Ru}, \mathrm{Os}$, and decrease as the ionic size of $\mathrm{M}^{2+}$ increases; ${ }^{28} \mathrm{Eu}_{2} \mathrm{RuH}_{6}$ and solid solution series $(\mathrm{Ca}, \mathrm{Eu})_{2} \mathrm{RuH}_{6}$ and $(\mathrm{Sr}, \mathrm{Eu})_{2} \mathrm{RuH}_{6}$ are paramagnetic with $\mu_{\text {eff }}=7.7 \mu_{\mathrm{B}}$, and magnetic order below $T_{\mathrm{C}}=29 \mathrm{~K} ;{ }^{.25}$ lowtemperature magnetic properties of $\mathrm{Eu}-\mathrm{Ru},(\mathrm{Ca}, \mathrm{Eu})-\mathrm{Ru}$, and (Sr,Eu)-Ru hydrides ${ }^{36}$ show ferromagnetic behavior; electric resistivities of $\mathrm{Mg}_{2} \mathrm{FeH}_{6}, \mathrm{Ca}_{2} \mathrm{RuH}_{6}, \mathrm{Sr}_{2} \mathrm{RuH}_{6}, \mathrm{Yb}_{2} \mathrm{RuH}_{6}$, and $\mathrm{Eu}_{2} \mathrm{RuH}_{6}$ indicate nonmetallic behavior; band structure calculations $^{37}$ predict band gaps that range from $1.3 \mathrm{eV}$ $\left(\mathrm{Sr}_{2} \mathrm{FeH}_{6}\right)$ to $4.0 \mathrm{eV}\left(\mathrm{Mg}_{2} \mathrm{OsH}_{6}\right)$ and increase in the sequence $\mathrm{Mg}_{2} \mathrm{TH}_{6}(\mathrm{~T}=\mathrm{Fe}, \mathrm{Ru}, \mathrm{Os})$ and decrease in the sequence $\mathrm{M}_{2} \mathrm{FeH}_{6}(\mathrm{M}=\mathrm{Mg}, \mathrm{Ca}, \mathrm{Sr}) ; \mathrm{Mg}_{2} \mathrm{FeH}_{6}, \mathrm{Ca}_{2} \mathrm{RuH}_{6}, \mathrm{Sr}_{2} \mathrm{RuH}_{6}$ are diamagnetic, or weakly paramagnetic; other properties of $\mathrm{Mg}_{2} \mathrm{FeH}_{6}$ : Mössbauer spectra ${ }^{26}$ consistent with low-spin $\mathrm{Fe}^{\mathrm{II}}$; hydrogen density $5.4 \mathrm{wt} \% ; 9.0 \times 10^{22} \mathrm{H}$ atoms $\mathrm{cm}^{-3}$; desorption enthalpy ${ }^{\mathbf{2 6}} \Delta H=98 \mathrm{~kJ} \mathrm{~mol}^{-1} \mathrm{H}_{2}$; INS, infrared, and Raman spectroscopies, ${ }^{38}$ has potential for thermochemical thermal energy storage, ${ }^{39}$ IR of Ru based hydrides; ${ }^{40}$ ordered structures (Figure 2) by npd on deuterides;, ${ }^{3,26-29} F m \overline{3} m$, $Z=4 ; \quad \mathrm{K}_{2} \mathrm{PtCl}_{6}$ type structure; octahedral $\left[\mathrm{TH}_{6}\right]^{4-} 18-$ electron complexes having T site symmetry $m \overline{3} m$; surrounded by $\mathrm{M}$ in eightfold cubic configuration corresponding to $\mathrm{CaF}_{2}$ type metal substructure; lif: $2 \mathrm{M}^{2+} \cdot\left[\mathrm{TH}_{6}\right]^{4-} ; \mathrm{T}=\mathrm{Fe}^{\mathrm{II}}, \mathrm{Ru}^{\mathrm{II}}$, $\mathrm{Os}^{\mathrm{II}} ; \mathrm{d}^{6}$. Interatomic distances are given in Table 2 .

\section{3 $\mathrm{Sr}_{2} \mathrm{IrH}_{5}\left(\mathrm{Mg}_{2} \mathrm{CoH}_{5}, \mathrm{Mg}_{2} \mathrm{IrH}_{5}, \mathrm{Ca}_{2} \mathrm{IrH}_{5}, \mathrm{Ca}_{2} \mathrm{RhH}_{5.4}\right.$, $\left.\mathrm{Sr}_{2} \mathrm{RhH}_{5.3}, \mathrm{Eu}_{2} \mathrm{IrH}_{5}\right)$}

$\mathrm{Sr}_{2} \mathrm{IrH}_{5}$ from Ir and $\mathrm{SrH}_{2}$ powder at $1033 \mathrm{~K}$ under hydrogen; ${ }^{41} \mathrm{Eu}_{2} \mathrm{IrD}_{5}$ from ${ }^{153} \mathrm{Eu}$ isotope enriched intermetallic $\mathrm{EuIr}_{2}$ at $673 \mathrm{~K}$ under deuterium, ${ }^{42}$ or by reaction of binary europium hydride (natural isotope mixture) with iridium metal 
Table 2 Interatomic distances $(\AA)$ in $\mathrm{Sr}_{2} \mathrm{RuD}_{6}$ and analogs

\begin{tabular}{llcccccc}
\hline & & $\mathrm{T}=\mathrm{Fe}$ & $\mathrm{T}=\mathrm{Ru}$ & $\mathrm{T}=\mathrm{Os}$ & & & $\mathrm{T}=\mathrm{Pt}$ \\
\hline $\mathrm{M}=\mathrm{Mg}$ & $\mathrm{T}-\mathrm{D}$ & 1.56 & 1.67 & 1.68 & $\mathrm{M}=\mathrm{Na}$ & $\mathrm{T}-\mathrm{D}$ & 1.62 \\
& $\mathrm{~T}-\mathrm{Mg}$ & 2.79 & 2.87 & 2.89 & & $\mathrm{~T}-\mathrm{Na}$ & 3.18 \\
& $\mathrm{Mg}-\mathrm{D}$ & 2.28 & 2.34 & 2.36 & & $\mathrm{Na}-\mathrm{D}$ & 2.60 \\
& $\mathrm{Mg}-\mathrm{Mg}$ & 3.22 & 3.31 & 3.34 & & $\mathrm{Na}-\mathrm{Na}$ & 3.67 \\
& $\mathrm{D}-\mathrm{D}$ & 2.20 & 2.32 & 2.34 & & $\mathrm{D}-\mathrm{D}$ & 2.28 \\
$\mathrm{M}=\mathrm{Ca}$ & $\mathrm{T}-\mathrm{D}$ & 1.62 & & 1.73 & $\mathrm{M}=\mathrm{K}$ & $\mathrm{T}-\mathrm{D}$ & 1.64 \\
& $\mathrm{~T}-\mathrm{Ca}$ & 3.05 & & 3.14 & & $\mathrm{~T}-\mathrm{K}$ & 3.62 \\
& $\mathrm{Ca}-\mathrm{D}$ & 2.49 & & 2.57 & & $\mathrm{~K}-\mathrm{D}$ & 2.90 \\
& $\mathrm{Ca}-\mathrm{Ca}$ & 3.52 & & 3.63 & & $\mathrm{~K}-\mathrm{K}$ & 4.07 \\
& $\mathrm{D}-\mathrm{D}$ & 2.29 & & 2.45 & & $\mathrm{D}-\mathrm{D}$ & 2.32 \\
$\mathrm{M}=\mathrm{Sr}$ & $\mathrm{T}-\mathrm{D}$ & & 1.69 & & $\mathrm{M}=\mathrm{Rb}$ & $\mathrm{T}-\mathrm{D}$ & 1.63 \\
& $\mathrm{~T}-\mathrm{Sr}$ & & 3.29 & & & $\mathrm{~T}-\mathrm{Rb}$ & 3.70 \\
& $\mathrm{Sr}-\mathrm{D}$ & & 2.69 & & & $\mathrm{Rb}-\mathrm{D}$ & 3.06 \\
& $\mathrm{Sr}-\mathrm{Sr}$ & & 3.80 & & $\mathrm{Rb}-\mathrm{Rb}$ & 4.27 \\
& $\mathrm{D}-\mathrm{D}$ & & 2.40 & & $\mathrm{D}-\mathrm{D}$ & 2.30 \\
$\mathrm{M}=\mathrm{Ba}$ & $\mathrm{T}-\mathrm{D}$ & & 1.72 & 1.78 & $\mathrm{M}=\mathrm{Cs}$ & $\mathrm{T}-\mathrm{D}$ & 1.64 \\
& $\mathrm{~T}-\mathrm{Ba}$ & & 3.47 & 3.48 & & $\mathrm{~T}-\mathrm{Cs}$ & 3.88 \\
& $\mathrm{Ba}-\mathrm{D}$ & & 2.85 & 2.85 & & $\mathrm{Cs}-\mathrm{D}$ & 3.23 \\
& $\mathrm{Ba}-\mathrm{Ba}$ & & 4.01 & 4.02 & & $\mathrm{Cs}-\mathrm{Cs}$ & 4.48 \\
& $\mathrm{D}-\mathrm{D}$ & & 2.44 & 2.51 & & $\mathrm{D}-\mathrm{D}$ & 2.32 \\
\hline
\end{tabular}

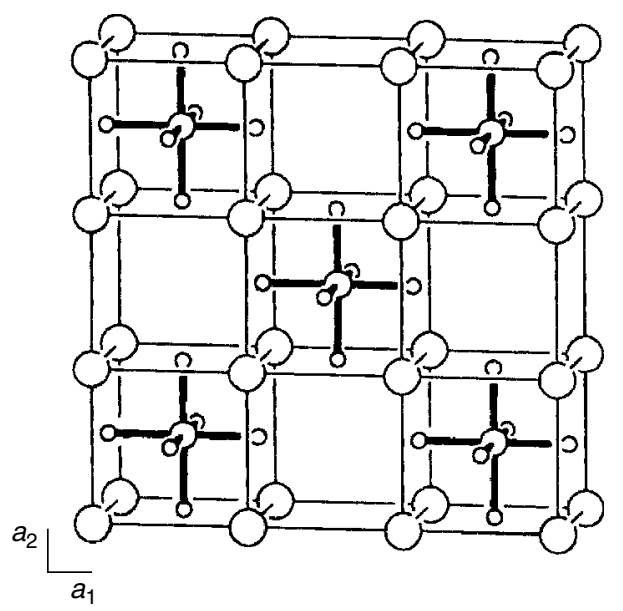

Figure 2 Structure of $\mathrm{Sr}_{2} \mathrm{RuD}_{6}$

at $970 \mathrm{~K}$ under deuterium pressure, ${ }^{43} \mathrm{Mg}_{2} \mathrm{CoH}_{5}$ by sintering metal powder mixtures at $620-770 \mathrm{~K}$ under $40-60 \mathrm{bar}$ hydrogen pressure; ${ }^{44}$ or by mechanical alloying followed by sintering; ${ }^{31}$ properties of $\mathrm{Mg}_{2} \mathrm{CoH}_{5}$ : black powder; electric resistivity consistent with nonmetallic behavior; band structure calculations $\mathrm{s}^{37}$ predict band gap of $1.9 \mathrm{eV}$, magnetization measurements indicate weak paramagnetism (possibly diamagnetism); ordered structure (Figure 3) from npd: ${ }^{44} P 4 / n m m, \quad c / a=1.477 ; \quad Z=1$; square-pyramidal $\left[\mathrm{CoH}_{5}\right]^{4-} 18$-electron complex with $\mathrm{T}$ site symmetry $4 \mathrm{~mm}$; surrounded by two $\mathrm{Mg}$ sites in eightfold nearly cubic configuration; two D sites, both ordered; transforms at $488 \mathrm{~K}$ into disordered cubic HT (high temperature) modification

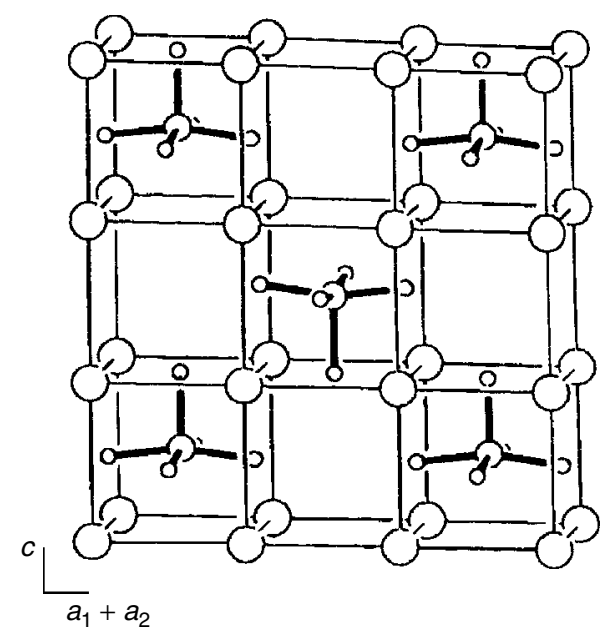

Figure 3 Structure of $\mathrm{Mg}_{2} \mathrm{CoD}_{5}$

similar to II; lif: $2 \mathrm{Mg}^{2+} \cdot\left[\mathrm{CoH}_{5}\right]^{4-} ; \mathrm{Co}^{\mathrm{I}} ; \mathrm{d}^{8} ; \mathrm{H}$ distribution in presumably isostructural $\mathrm{Mg}_{2} \mathrm{IrH}_{5}$ not yet confirmed; hydrogen density: $4.5 \mathrm{wt} \%, 7.5 \times 10^{22} \mathrm{H}$ atoms $\mathrm{cm}^{-3}$; desorption enthalpy $\Delta H=86 \mathrm{~kJ} \mathrm{~mol}^{-1} \mathrm{H}_{2}$; decomposes into $\delta$ - $\mathrm{MgCo}$ and $\mathrm{MgH}_{2} ;{ }^{45} \mathrm{Sr}_{2} \mathrm{IrD}_{5}$ : partially disordered structure from $\mathrm{npd}^{41}$ at $4.2 \mathrm{~K}: I 4 / \mathrm{mmm}, c / a=1.465, Z=2$; Ir surrounded by five $\mathrm{D}$ in a partially disordered average octahedral configuration having 4/mmm site symmetry; equatorial D sites fully and apical D sites half occupied; transforms between 140 and $200 \mathrm{~K}$ into disordered cubic HT modification similar to II; $\mathrm{Ca}_{2} \mathrm{RhD}_{5.4}$ and $\mathrm{Sr}_{2} \mathrm{RhD}_{5.3}$ with higher occupancy factor $0.89(2),{ }^{\mathbf{4 6 , 4 7}}$ disordered cubic structure by npd: $F m \overline{3} m, Z=4$, no phase transition; $\mathrm{Eu}_{2} \mathrm{IrD}_{5}$, 
Table 3 Interatomic distances $(\AA)$ in $\mathrm{Sr}_{2} \mathrm{IrD}_{5}$ and analogs

\begin{tabular}{|c|c|c|c|c|c|c|c|}
\hline & & \multicolumn{3}{|c|}{ Cubic HT-phases } & \multicolumn{3}{|c|}{ Tetragonal LT-phases } \\
\hline & & $\mathrm{T}=\mathrm{Co}$ & $\mathrm{T}=\mathrm{Rh}$ & $\mathrm{T}=\mathrm{Ir}$ & $\mathrm{T}=\mathrm{Co}$ & $\mathrm{T}=\mathrm{Rh}$ & $\mathrm{T}=\mathrm{Ir}$ \\
\hline \multirow[t]{5}{*}{$\mathrm{M}=\mathrm{Mg}$} & $\mathrm{T}-\mathrm{D}$ & 1.53 & & & $\begin{array}{l}(4 \times) 1.51 \\
(1 \times) 1.59\end{array}$ & & \\
\hline & $\mathrm{T}-\mathrm{Mg}$ & 2.79 & & & 2.75 & & \\
\hline & $\mathrm{Mg}-\mathrm{D}$ & 2.28 & & & 2.23 & & \\
\hline & $\mathrm{Mg}-\mathrm{Mg}$ & 3.23 & & & 3.16 & & \\
\hline & $\mathrm{D}-\mathrm{D}$ & 2.16 & & & 2.12 & & \\
\hline \multirow[t]{5}{*}{$\mathrm{M}=\mathrm{Ca}$} & $\mathrm{T}-\mathrm{D}$ & & 1.75 & 1.70 & & & $\begin{array}{c}(4 \times) 1.68 \\
\left(2 \times, \text { dis }^{a}\right) 1.81\end{array}$ \\
\hline & $\mathrm{T}-\mathrm{Ca}$ & & 3.14 & 3.14 & & & 3.13 \\
\hline & $\mathrm{Ca}-\mathrm{D}$ & & 2.57 & 2.56 & & & 2.52 \\
\hline & $\mathrm{Ca}-\mathrm{Ca}$ & & 3.63 & 3.62 & & & 3.56 \\
\hline & $\mathrm{D}-\mathrm{D}$ & & 2.48 & 2.41 & & & 2.38 \\
\hline \multirow[t]{5}{*}{$\mathrm{M}=\mathrm{Sr}$} & $\mathrm{T}-\mathrm{D}$ & & 1.73 & 1.71 & & & $\begin{array}{c}(4 \times) 1.69 \\
(2 \times, \text { dis. }) 1.82\end{array}$ \\
\hline & $\mathrm{T}-\mathrm{Sr}$ & & 3.28 & 3.31 & & & 3.30 \\
\hline & $\mathrm{Sr}-\mathrm{D}$ & & 2.69 & 2.71 & & & 2.66 \\
\hline & $\mathrm{Sr}-\mathrm{Sr}$ & & 3.79 & 3.82 & & & 3.76 \\
\hline & $\mathrm{D}-\mathrm{D}$ & & 2.45 & 2.42 & & & 2.39 \\
\hline \multirow[t]{5}{*}{$\mathrm{M}=\mathrm{Eu}$} & $\mathrm{T}-\mathrm{D}$ & & & 1.69 & & & $\begin{array}{c}(4 \times) 1.70 \\
(2 \times, \text { dis. }) 1.75\end{array}$ \\
\hline & $\mathrm{T}-\mathrm{Eu}$ & & & 3.28 & & & 3.27 \\
\hline & $\mathrm{Eu}-\mathrm{D}$ & & & 2.68 & & & 2.64 \\
\hline & $\mathrm{Eu}-\mathrm{Eu}$ & & & 3.78 & & & 3.73 \\
\hline & D-D & & & 2.39 & & & 2.40 \\
\hline
\end{tabular}

${ }^{\mathrm{a} D i s o r d e r e d .}$

$\mathrm{Ca}_{2} \mathrm{IrD}_{5}$, and solid solutions $(\mathrm{Ca}, \mathrm{Eu})_{2} \mathrm{IrD}_{5},(\mathrm{Sr}, \mathrm{Eu})_{2} \mathrm{IrD}_{5}$ : disordered structure by npd at room temperature: ${ }^{\mathbf{4 2}} \mathrm{Fm} \overline{3} \mathrm{~m}$, $Z=4$; Ir surrounded by five $\mathrm{D}$ atoms in a disordered average octahedral configuration similar to II; transition occurs between 200 and $275 \mathrm{~K}$ to partially ordered tetragonal modification $I 4 / \mathrm{mmm}$ similar to $\mathrm{Sr}_{2} \mathrm{IrD}_{5} ;{ }^{43}$ paramagnetic with $\mu_{\text {eff }}=7.3 \mu_{\mathrm{B}}$; ferromagnetic below $T_{\mathrm{C}}=20 \mathrm{~K}$; rhodiumbased ' $\mathrm{Mg}_{2} \mathrm{RhH}_{5}$ ' analog not obtained, presumably because of the formation of competing hydride $\mathrm{Mg}_{2} \mathrm{RhH}_{1.1}$ (filled $\mathrm{Ti}_{2} \mathrm{Ni}$ type structure) and binary $\mathrm{Mg}_{2} \mathrm{Rh}$ ( $\mathrm{Ti}_{2} \mathrm{Pd}$ type structure). ${ }^{48}$ Other Rh compounds known: ${ }^{49} \mathrm{Eu}_{2} \mathrm{RhH}_{5}$ and solid solutions $(\mathrm{Sr}, \mathrm{Eu})_{2} \mathrm{RhH}_{5}$. Interatomic distances are given in Table 3.

\section{4 $\mathrm{Na}_{2} \mathrm{PtH}_{4}\left(\mathrm{~K}_{2} \mathrm{PdH}_{4}, \mathrm{Na}_{2} \mathrm{PdH}_{4}\right)$}

$\mathrm{Na}_{2} \mathrm{PtH}_{4}$ by sintering $\mathrm{NaH}$ and $\mathrm{Pt}$ at about $573 \mathrm{~K}$ under hydrogen ${ }^{\mathbf{5 0}}$ red-violet powder; diamagnetic from susceptibility measurements; $\mathrm{K}_{2} \mathrm{PdH}_{4}$ by heating $\mathrm{KH}$ with $\mathrm{Pd}$ at $610 \mathrm{~K}$ under 16 bar hydrogen pressure; ${ }^{51}$ yellow-green powder, nonmetallic; band gap of $1.2 \mathrm{eV}$ predicted from theoretical band structure calculations; ${ }^{\mathbf{5 2}}$ decomposes before melting; $\mathrm{Na}_{2} \mathrm{PdH}_{4}$ from $\mathrm{NaH}$ and $\mathrm{Pd}$ at up to 2500 bar and $770 \mathrm{~K} ;{ }^{\mathbf{5 3}}$ ordered structures (Figure 4 ) from npd on deuterides: $I 4 / \mathrm{mmm}, \mathrm{Z}=2$; square-planar $\left[\mathrm{PtH}_{4}\right]^{2-}\left(\right.$ or $\left.\left[\mathrm{PdH}_{4}\right]^{2-}\right) 16-$ electron complexes with T site symmetry $4 / \mathrm{mmm}$; surrounded by $\mathrm{Na}(\mathrm{K})$ in eightfold cubic configuration; one $\mathrm{D}$ site; $\mathrm{Na}_{2} \mathrm{PtH}_{4}$ transforms at about $573 \mathrm{~K}$ into disordered cubic

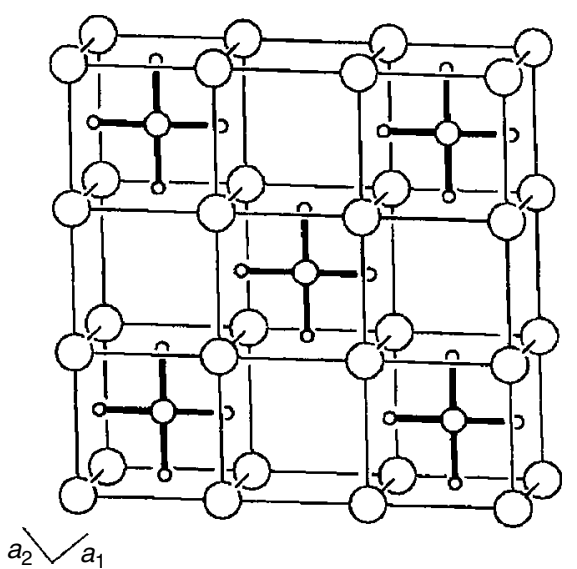

Figure 4 Structure of $\mathrm{Na}_{2} \mathrm{PtD}_{4}$

HT modification similar to II; $\mathrm{Na}_{2} \mathrm{PdH}_{4}$ does not transform up to $600 \mathrm{~K}$ and decomposes into $\mathrm{Na}_{2} \mathrm{PdH}_{2},{ }^{.53}$ lif: $2 \mathrm{M}^{+}$. $\left[\mathrm{TH}_{4}\right]^{2-}$; $\mathrm{T}=\mathrm{Pd}^{\mathrm{II}}, \mathrm{Pt}^{\mathrm{II}}, \mathrm{d}^{8}$. Interatomic distances are given in Table 4 .

\section{5 $\mathrm{Mg}_{2} \mathrm{NiH}_{4}$}

From binary alloy $\mathrm{Mg}_{2} \mathrm{Ni}$ by hydrogenation at temperatures above $623 \mathrm{~K},{ }^{\mathbf{5}}$ or by sintering powder mixtures of the elements at $723-773 \mathrm{~K}$ under 90 bar hydrogen pressure, ${ }^{30}$ fine powder 
Table 4 Interatomic distances $(\AA)$ in $\mathrm{Na}_{2} \mathrm{PtD}_{4}$ and analogs

\begin{tabular}{llcr}
\hline & & $\mathrm{T}=\mathrm{Pd}$ & $\mathrm{T}=\mathrm{Pt}$ \\
\hline $\mathrm{M}=\mathrm{Na}$ & $\mathrm{T}-\mathrm{D}$ & 1.61 & 1.64 \\
& T-Na & 3.14 & 3.12 \\
& Na-D & 2.52 & 2.52 \\
& Na-Na & 3.31 & 3.38 \\
& $\mathrm{D}-\mathrm{D}$ & 2.27 & 2.32 \\
$\mathrm{M}=\mathrm{K}$ & $\mathrm{T}-\mathrm{D}$ & 1.62 & \\
& T-K & 3.49 & \\
& K-D & 2.85 & \\
& K-K & 3.84 & \\
& D-D & 2.30 & \\
\hline
\end{tabular}

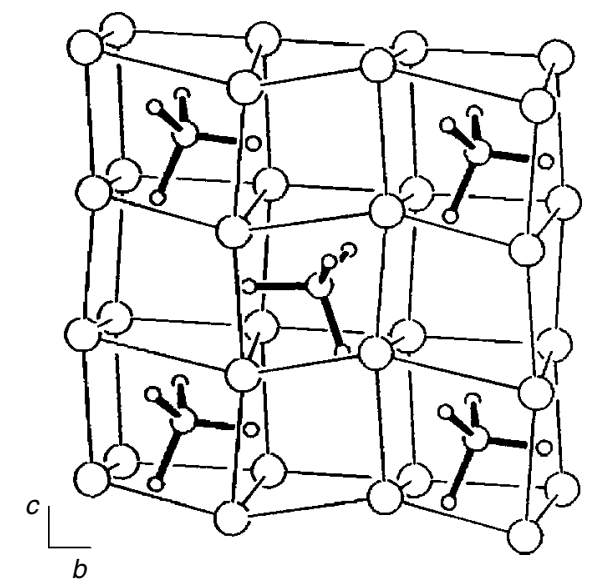

Figure 5 Structure of $\mathrm{Mg}_{2} \mathrm{NiD}_{4}$

of dark-red (fully hydrided) or brownish (partially hydrided) color; stable in air; diamagnetic, nonmetallic; theoretical band structure calculations ${ }^{37}$ on disordered structure model predict band gap of $1.8 \mathrm{eV}$; vibrational spectroscopy; ${ }^{\mathbf{5 4}}$ bonding and stability investigated by $\mathrm{ab}$ initio density functional calculations; ${ }^{14,55} \mathrm{H}$ density: $3.6 \mathrm{wt} \%, 5.8 \times 10^{22} \mathrm{H}$ atoms $\mathrm{cm}^{-3}$; desorption enthalpy $\Delta H=64 \mathrm{~kJ} \mathrm{~mol}^{-1} \mathrm{H}_{2}$; ordered structure (Figure 5) by high-resolution npd on deuteride: ${ }^{6}$ $C 2 / \mathrm{c}, Z=8$; tetrahedral $\left[\mathrm{NiH}_{4}\right]^{4-}$ 18-electron complex having symmetry 1 ; surrounded by three $\mathrm{Mg}$ sites in eightfold, strongly distorted cubic configuration; four D sites; Xray and electron microscopy evidence for microtwinning ${ }^{\mathbf{5 6}}$ parallel to $b c$ plane; transforms between 483 and $513 \mathrm{~K}$ into disordered cubic HT modification similar to $\mathrm{II},{ }^{\mathbf{5 7}}$ influenced by mechanical grinding; ${ }^{58}$ lif: $2 \mathrm{Mg}^{2+} \cdot\left[\mathrm{NiH}_{4}\right]^{4-} ; \mathrm{Ni}^{0}, \mathrm{~d}^{10}$.

Distances $(\AA)$ and angles: $\mathrm{Ni}-\mathrm{D}=1.52-1.57$; $\mathrm{D}-\mathrm{Ni}-\mathrm{D}=$ $104-119^{\circ} ; \mathrm{Mg}-\mathrm{Ni}=2.65 ; \mathrm{Ni}-\mathrm{Ni}=4.30 ; \mathrm{Mg}-\mathrm{Mg}=3.10$; $\mathrm{Mg}-\mathrm{D}=1.97 ; \mathrm{D}-\mathrm{D}=2.42$.

\section{6 $\mathrm{K}_{2} \mathrm{PtH}_{4}\left(\mathrm{Rb}_{2} \mathrm{PtH}_{4}, \mathrm{Cs}_{2} \mathrm{PtH}_{4}, \mathrm{Rb}_{2} \mathrm{PdH}_{4}, \mathrm{Cs}_{2} \mathrm{PdH}_{4}\right)$}

From binary hydrides and $\mathrm{Pt}(\mathrm{Pd})$ sponge at 593-693 K under 0.8 bar ( $\mathrm{Pd}$ compounds 20 bar) hydrogen pressure; ${ }^{\mathbf{5 9}}$

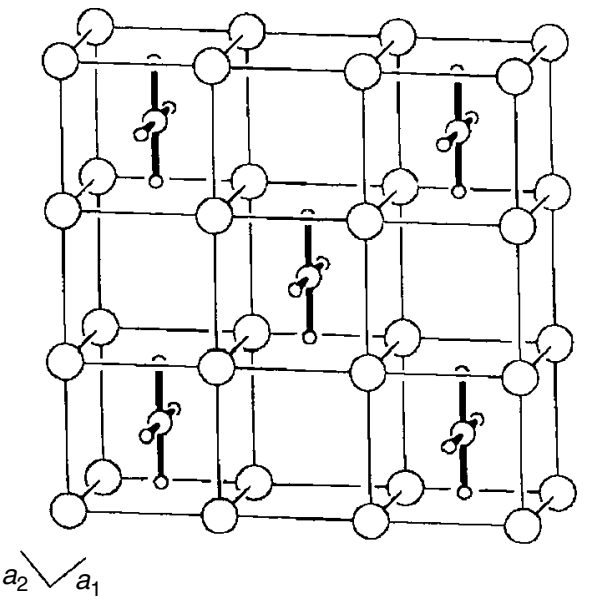

Figure 6 Structure of $\mathrm{K}_{2} \mathrm{PtD}_{4}$

pyrophoric, colorless powders; sensitive to moisture and air; $\mathrm{K}_{2} \mathrm{PtH}_{4}$ : diamagnetic; $\mathrm{NMR}$ data at room temperature consistent with rotational disorder of $\mathrm{PtH}_{4}$ groups; structures from npd on deuterides at 295 and $15 \mathrm{~K}\left(\mathrm{~K}_{2} \mathrm{PtD}_{4}, \mathrm{Rb}_{2} \mathrm{PdD}_{4}\right.$, $\left.\mathrm{Cs}_{2} \mathrm{PdD}_{4}\right), 16 \mathrm{~K}\left(\mathrm{Rb}_{2} \mathrm{PtD}_{4}\right)$, and $12 \mathrm{~K}\left(\mathrm{Cs}_{2} \mathrm{PtD}_{4}\right)$; disordered room temperature structure: cubic, $F m \overline{3} m, Z=4, \mathrm{CaF}_{2}$ type metal substructure similar to II; transforms at $250 \mathrm{~K}$

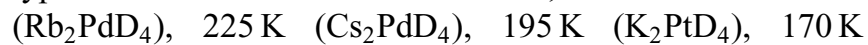
$\left(\mathrm{Rb}_{2} \mathrm{PtD}_{4}\right)$, and $150 \mathrm{~K}\left(\mathrm{Cs}_{2} \mathrm{PtD}_{4}\right)$, into ordered tetragonal low-temperature (LT) structure (Figure 6): $P 4_{2} / m n m, Z=2$; planar $\left[\mathrm{PtH}_{4}\right]^{2-}\left(\right.$ or $\left.\left[\mathrm{PdH}_{4}\right]^{2-}\right)$ 16-electron complexes with $\mathrm{T}$ site symmetry m.mm; unlike $\mathrm{Na}_{2} \mathrm{PtH}_{4}$, these complexes are oriented perpendicular to the tetragonal base; surrounded by $\mathrm{M}^{+}$in eightfold, nearly cubic configuration; two D sites; lif: $2 \mathrm{M}^{+} .\left[\mathrm{TH}_{4}\right]^{2-} ; \mathrm{T}=\mathrm{Pd}^{\mathrm{II}}, \mathrm{Pt}^{\mathrm{II}}, \mathrm{d}^{8}$. Interatomic distances are given in Table 5 .

\section{7 $\quad \mathrm{Na}_{2} \mathrm{PdH}_{2}\left(\mathrm{Li}_{2} \mathrm{PdH}\right)_{2}$}

$\mathrm{Na}_{2} \mathrm{PdH}_{2}$ from $\mathrm{NaH}$ with $\mathrm{Pd}$ at $643 \mathrm{~K}$ under 50 bar hydrogen pressure; ${ }^{\mathbf{6 0}}$ brittle solid; single crystals show metallic luster; melts at $681 \mathrm{~K}$ without decomposing; metallic conductivity measured on single crystals and semiconducting behavior on powder; $\mathrm{Li}_{2} \mathrm{PdH}_{2}{ }^{\mathbf{5 1}}$ from $\mathrm{LiH}$ and $\mathrm{Pd}$ at $680 \mathrm{~K}$; melts at $773 \mathrm{~K}$; electronic band structure calculations ${ }^{52,61}$ predict filled $\mathrm{d}$ bands and metallic properties in two dimensions ( $a b$ plane), originating from overlap between $\mathrm{Pd}$ d-block bands and alkali metal s- and p-block bands; $\mathrm{Na}_{2} \mathrm{PdH}_{2}$ found to be metallic; ${ }^{61}$ ordered structure of $\mathrm{Na}_{2} \mathrm{PdH}_{2}$ (Figure 7) from Xray single-crystal diffraction on hydride and npd on deuteride: I4/mmm, $\mathrm{Z}=2 ; \mathrm{Na}_{2} \mathrm{HgO}_{2}$ type structure; linear $\left[\mathrm{PdH}_{2}\right]^{2-} 14$ electron complexes centered on site having 4/mmm symmetry; surrounded by $\mathrm{Na}$ in 10-fold bicapped cubic configuration; one D site; lif: $2 \mathrm{Na}^{+} \cdot\left[\mathrm{PdH}_{2}\right]^{2-} ; \mathrm{Pd}^{0}, \mathrm{~d}^{10} ;$ structure of $\mathrm{Li}_{2} \mathrm{PdH}_{2}$ from $\mathrm{npd},{ }^{51}$ evidence for linear $\left[\mathrm{PtH}_{2}\right]^{2-}$ complexes in quaternary $\mathrm{Na}_{2} \mathrm{PdH}_{2}-\mathrm{Na}_{2} \mathrm{PtH}_{4}$ system. ${ }^{62}$ 
Table 5 Interatomic distances $(\AA)$ in $\mathrm{K}_{2} \mathrm{PtD}_{4}$ and analogs

\begin{tabular}{|c|c|c|c|c|c|}
\hline & & \multicolumn{2}{|c|}{ Cubic HT-phases } & \multicolumn{2}{|c|}{ Tetragonal LT-phases } \\
\hline & & $\mathrm{T}=\mathrm{Pd}$ & $\mathrm{T}=\mathrm{Pt}$ & $\mathrm{T}=\mathrm{Pd}$ & $\mathrm{T}=\mathrm{Pt}$ \\
\hline \multirow[t]{5}{*}{$\mathrm{M}=\mathrm{K}$} & $\mathrm{T}-\mathrm{D}$ & & 1.62 & & $\begin{array}{l}(2 \times) 1.58 \\
(2 \times) 1.59\end{array}$ \\
\hline & $\mathrm{T}-\mathrm{K}$ & & 3.47 & & 3.45 \\
\hline & $\mathrm{K}-\mathrm{D}$ & & 2.86 & & 2.83 \\
\hline & $\mathrm{K}-\mathrm{K}$ & & 4.01 & & 3.95 \\
\hline & D-D & & 2.29 & & 2.24 \\
\hline \multirow[t]{6}{*}{$\mathrm{M}=\mathrm{Rb}$} & $\mathrm{T}-\mathrm{D}$ & 1.63 & 1.63 & $(2 \times) 1.67$ & $(2 \times) 1.60$ \\
\hline & & & & $(2 \times) 1.67$ & $(2 \times) 1.69$ \\
\hline & $\mathrm{T}-\mathrm{Rb}$ & 3.64 & 3.63 & 3.61 & 3.60 \\
\hline & $\mathrm{Rb}-\mathrm{D}$ & 3.01 & 3.00 & 2.95 & 2.95 \\
\hline & $\mathrm{Rb}-\mathrm{Rb}$ & 4.21 & 4.19 & 4.12 & 4.14 \\
\hline & $\mathrm{D}-\mathrm{D}$ & 2.31 & 2.31 & 2.36 & 2.33 \\
\hline \multirow[t]{6}{*}{$\mathrm{M}=\mathrm{Cs}$} & $\mathrm{T}-\mathrm{D}$ & 1.60 & 1.61 & $(2 \times) 1.59$ & $(2 \times) 1.58$ \\
\hline & & & & $(2 \times) 1.64$ & $(2 \times) 1.61$ \\
\hline & $\mathrm{T}-\mathrm{Cs}$ & 3.84 & 3.80 & 3.80 & 3.75 \\
\hline & Cs-D & 3.20 & 3.15 & 3.14 & 3.12 \\
\hline & $\mathrm{Cs}-\mathrm{Cs}$ & 4.44 & 4.38 & 4.36 & 4.32 \\
\hline & D-D & 2.26 & 2.28 & 2.28 & 2.25 \\
\hline
\end{tabular}

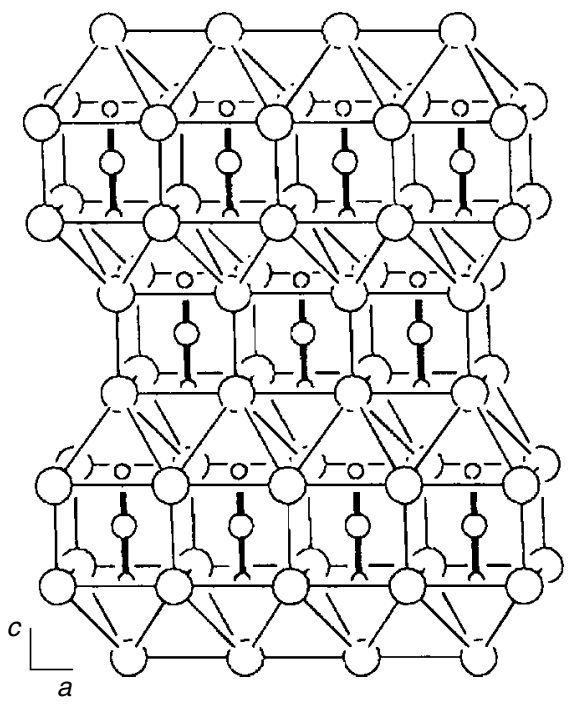

Figure 7 Structure of $\mathrm{Na}_{2} \mathrm{PdD}_{2}$

Distances $(\AA):$ Pd-D = $(2 \times) 1.68(\mathrm{Na}),(2 \times) 1.67(\mathrm{Li})$; $\mathrm{Pd}-\mathrm{Na}(\mathrm{Li})=3.13(2.64) ; \mathrm{Pd}-\mathrm{Pd}=3.60(\mathrm{Na}), 3.11(\mathrm{Li})$; $\mathrm{Na}(\mathrm{Li})-\mathrm{D}=2.42(2.03) ; \mathrm{D}-\mathrm{D}=3.35(\mathrm{Na}), 2.85(\mathrm{Li})$.

\section{8 $\mathrm{K}_{3} \mathrm{PtH}_{5}\left(\mathrm{Rb}_{3} \mathrm{PtH}_{5}, \mathrm{Cs}_{3} \mathrm{PtH}_{5}, \mathrm{~K}_{3} \mathrm{PdH}_{5}, \mathrm{Rb}_{3} \mathrm{PdH}_{5}\right.$, $\left.\mathrm{Cs}_{3} \mathrm{PdH}_{5}\right)$}

From binary alkali hydrides and platinum (palladium) sponge at $573-623 \mathrm{~K}$ under $0.8 \mathrm{bar}$ ( $\mathrm{Pd}$ compounds $70 \mathrm{bar}$ ) hydrogen; ${ }^{63}$ colorless powders, sensitive to air and moisture; ordered structures (Figure 8 ) by npd on deuterides: $P 4 / \mathrm{mbm}$, $Z=2$; isostructural to $\mathrm{Rb}_{3} \mathrm{PdF}_{5}$; planar $\left[\mathrm{PtH}_{4}\right]^{2-}$ (or

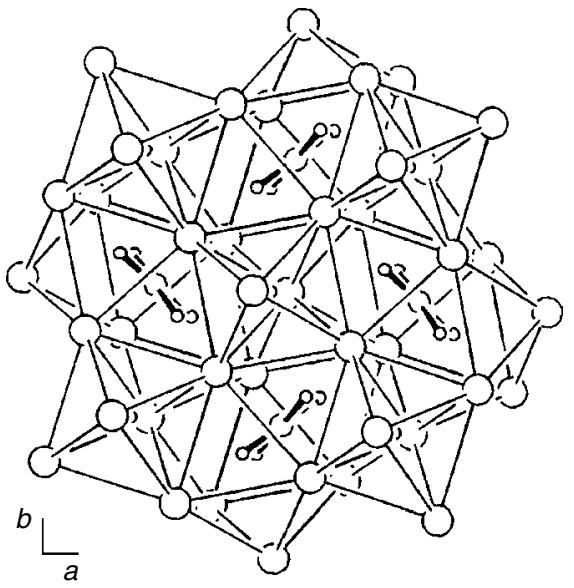

Figure 8 Structure of $\mathrm{K}_{3} \mathrm{PtD}_{5}$

$\left[\mathrm{PdH}_{4}\right]^{2-}$ ) 16-electron complexes with $\mathrm{T}$ site symmetry m.mm; surrounded by two $\mathrm{M}$ sites in irregular ninefold configuration; two D sites, of which the twofold is octahedral surrounded by $\mathrm{M}^{+}$only; lif: $3 \mathrm{M}^{+} \cdot\left[\mathrm{TH}_{4}\right]^{2-} \cdot \mathrm{H}^{-} ; \mathrm{T}=\mathrm{Pd}^{\mathrm{II}}$, $\mathrm{Pt}^{\mathrm{II}}, \mathrm{d}^{8} ; \mathrm{K}_{3} \mathrm{PtH}_{5}$ decomposes at $673 \mathrm{~K}$ into $\mathrm{K}_{2} \mathrm{PtH}_{4}$ and $\mathrm{KH}$; $\mathrm{Rb}_{3} \mathrm{PtH}_{5}$ and $\mathrm{Cs}_{3} \mathrm{PtH}_{5}$ transform reversibly above 615 and $465 \mathrm{~K}$, respectively, into disordered cubic HT modifications with $\mathrm{Cu}_{3} \mathrm{Au}$ type metal substructure $(P m \overline{3} m, Z=1 ; \mathrm{H}$ positions not refined); no evidence for such transitions in $\mathrm{Pd}$ compounds; ordered substitution on $\mathrm{M}$ sites leads to quaternary hydrides $\mathrm{K}_{2} \mathrm{RbPtH}_{5}$ and $\mathrm{K}_{2} \mathrm{CsPtH}_{5}$ (H positions not refined). Interatomic distances are given in Table 6.

\section{9 $\mathrm{K}_{3} \mathrm{PdH}_{3}\left(\mathrm{Rb}_{3} \mathrm{PdH}_{3}, \mathrm{Cs}_{3} \mathrm{PdH}_{3}\right)$}

From $\mathrm{KH}$ and $\mathrm{Pd}$ sponge at $623 \mathrm{~K}$ under hydrogen; yellow powder at room temperature, orange-red at high temperature, ${ }^{\mathbf{6 4}}$ ordered structure (Figure 9) from npd on deuteride: $P 4_{2} / \mathrm{mnm}$, $Z=8$; three $\mathrm{Pd}$ sites with ratios $\mathrm{Pd} 1: \operatorname{Pd} 2: \operatorname{Pd} 3=1: 1: 2$, each forming a linear $\left[\mathrm{PdH}_{2}\right]^{2-}$ 14-electron complex having

Table 6 Interatomic distances $(\AA)$ in $\mathrm{K}_{3} \mathrm{PtD}_{5}$ and analogs

\begin{tabular}{clccc}
\hline & & $\mathrm{M}=\mathrm{K}$ & $\mathrm{M}=\mathrm{Rb}$ & $\mathrm{M}=\mathrm{Cs}$ \\
\hline $\mathrm{T}=\mathrm{Pd}$ & $\mathrm{Pd}-\mathrm{D}$ & 1.63 & 1.61 & 1.64 \\
& $\mathrm{Pd}-\mathrm{M}$ & 3.46 & 3.62 & 3.67 \\
& $\mathrm{M}-\mathrm{D}$ & 2.84 & 2.99 & 3.04 \\
& $\mathrm{M}-\mathrm{D}-$ & 2.73 & 2.85 & 3.01 \\
$\mathrm{~T}=\mathrm{Pt}$ & 3.74 & 4.03 & 4.20 \\
& $\mathrm{M}-\mathrm{M}$ & 5.26 & 5.52 & 5.87 \\
& $\mathrm{Pd}-\mathrm{Pd}$ & 2.20 & 2.17 & 2.30 \\
& $\mathrm{D}-\mathrm{D}$ & 1.63 & 1.58 & 1.64 \\
& $\mathrm{Pt}-\mathrm{D}$ & 3.47 & 3.57 & 3.68 \\
& $\mathrm{Pt}-\mathrm{M}$ & 2.74 & 2.98 & 3.03 \\
& $\mathrm{M}-\mathrm{D}$ & 2.72 & 2.86 & 2.96 \\
& $\mathrm{M}-\mathrm{D}-$ & 3.85 & 4.05 & 4.25 \\
& $\mathrm{M}-\mathrm{M}$ & 5.28 & 5.54 & 5.90 \\
& $\mathrm{Pt}-\mathrm{Pt}$ & 2.23 & 2.14 & 2.25 \\
\hline
\end{tabular}




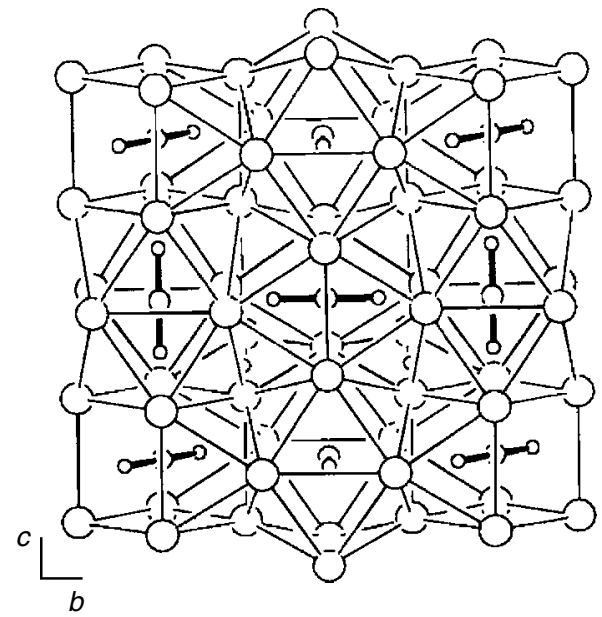

Figure 9 Structure of $\mathrm{K}_{3} \mathrm{PdD}_{3}$

respectively $m . m m, m . m m$, and $2 / m$ environments; surrounded by three $\mathrm{K}$ sites in 12-fold configurations; four D sites, of which one eightfold is coordinated by $\mathrm{K}^{+}$in oct configuration; lif: $3 \mathrm{~K}^{+} \cdot\left[\mathrm{PdH}_{2}\right]^{2-} \cdot \mathrm{H}^{-} ; \mathrm{Pd}^{0}, \mathrm{~d}^{10} ; \mathrm{X}$-ray evidence ${ }^{59,63}$ for isostructural $\mathrm{Cs}_{3} \mathrm{PdH}_{3}$ and $\mathrm{Rb}_{3} \mathrm{PdH}_{3} ; \mathrm{K}_{3} \mathrm{PdH}_{3}$ transforms at $\sim 473 \mathrm{~K}$ into disordered HT modification $(P m \overline{3} \mathrm{~m})$ with cubic $\mathrm{Cu}_{3} \mathrm{Au}$ type metal substructure while $\mathrm{Rb}_{3} \mathrm{PdH}_{3}$ transforms into $\operatorname{Im} \overline{3}$ HT modification.

Distances $(\AA): \mathrm{Pd}-\mathrm{D}=(2 \times) 1.68(\mathrm{Pd} 1),(2 \times) 1.68(\mathrm{Pd} 2)$, $(2 \times) 1.69(\mathrm{Pd} 3) ; \mathrm{Pd}-\mathrm{K}=3.04 ; \mathrm{K}-\mathrm{D}=2.64, \mathrm{~K}-\mathrm{D}^{-}=2.74$, $\mathrm{D}-\mathrm{D}=3.37$.

\subsection{0 $\mathrm{CaPdH}_{2}\left(\mathrm{SrPdH}_{2.7}, \mathrm{EuPdH}_{3}, \mathrm{YbPdH}_{2.7}, \mathrm{CaNiH}_{3}\right.$, $\mathrm{YbNiH}_{2.7}$ )}

$\mathrm{CaPdH}_{2}$ from $\mathrm{CaH}_{2}$ and $\mathrm{Pd}$ sponge at $1123 \mathrm{~K}$ under hydrogen pressures up to 300 bar; $^{65}$ disordered structure (Figure 10) from npd on deuteride at 295 and $10 \mathrm{~K}: P m \overline{3} m, Z=1$; Pd and $\mathrm{Ca}$ form CsCl-type metal substructure as in binary $\mathrm{CaPd}$; disordered D site near anion positions of perovskite-type structure with $2 / 3$ occupancy and large displacement amplitudes; structure model consistent with orientationally disordered linear $\left[\mathrm{PdH}_{2}\right]^{2-}$ 14-electron complexes; no evidence for D ordering down to $10 \mathrm{~K}$; lif: $\mathrm{Ca}^{2+} \cdot\left[\mathrm{PdH}_{2}\right]_{\mathrm{av}}{ }^{2-} ; \mathrm{Pd}^{0}, \mathrm{~d}^{10} ; \mathrm{X}$-ray evidence for rhombohedral distortion at room temperature; ${ }^{\mathbf{6 6}}$ copperred $\mathrm{SrPdH}_{2.7}$ by hydrogenation of $\mathrm{Sr}-\mathrm{Pd}$ alloy; ${ }^{67}$ structure of deuteride from npd: D site occupancy 0.91; black $\mathrm{EuPdH}_{3}$ by hydrogenation of intermetallic EuPd (with CrB type structure) at 15 bar hydrogen pressure at $500 \mathrm{~K} ;{ }^{\mathbf{6 8}}$ structure of deuteride from npd: full D site occupancy; metallic conductor; orders ferromagnetic at $21 \mathrm{~K}$; X-ray evidence for presumably isostructural $\mathrm{YbPdH}_{2.7},{ }^{69} \mathrm{YbNiH}_{2.7}{ }^{69}$ and $\mathrm{CaNiH}_{3}{ }^{70}$ Electronic band structure calculations on stoichiometric $\mathrm{APdH}_{3}$ $(\mathrm{A}=\mathrm{Sr}, \mathrm{Eu}, \mathrm{Yb})$ predict metallic properties. ${ }^{71}$ Interatomic distances are given in Table 7.

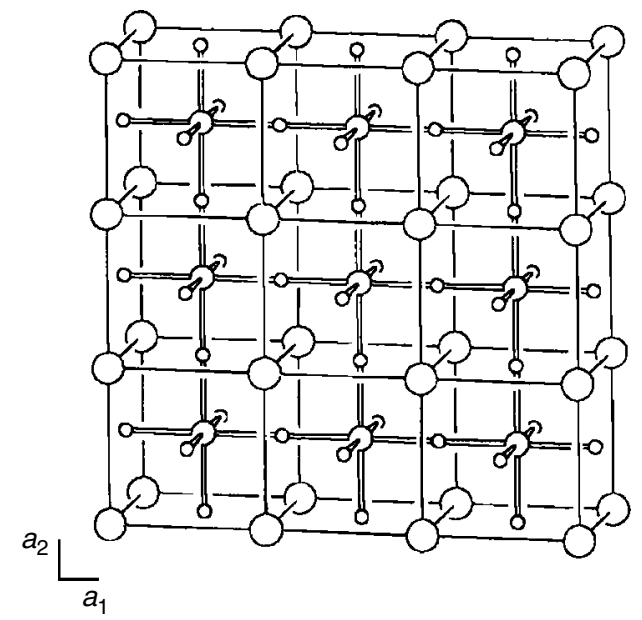

Figure 10 Structure of $\mathrm{CaPdD}_{2}$

Table 7 Interatomic distances $(\AA)$ in $\mathrm{CaPdD}_{2}$ and analogs

\begin{tabular}{lccc}
\hline & $\mathrm{M}=\mathrm{Ca}$ & $\mathrm{M}=\mathrm{Sr}$ & $\mathrm{M}=\mathrm{Eu}$ \\
\hline Pd-D & 1.84 & 1.92 & 1.90 \\
Pd-M & 3.19 & 3.33 & 3.29 \\
M-D & 2.61 & 2.72 & 2.69 \\
M-M & 3.69 & 3.84 & 3.80 \\
D-D & 2.61 & 2.72 & 2.69 \\
\hline
\end{tabular}

\section{$3.11 \mathrm{Li}_{4} \mathrm{RuH}_{6}\left(\mathrm{Na}_{4} \mathrm{RuH}_{6}, \mathrm{Li}_{4} \mathrm{OsH}_{6}\right)$}

From reactions of binary hydrides $\mathrm{MH}$ with $\mathrm{T}$ at $753 \mathrm{~K}$ and 10 bar hydrogen pressure, ${ }^{\mathbf{7 2}}$ no compound formation with $\mathrm{KH}$ and $\mathrm{Fe}$ up to $1073 \mathrm{~K}$ and 100 bar; $\mathrm{Ru}$ does not react with $\mathrm{KH}$, and Os not with $\mathrm{NaH}$ and $\mathrm{KH}$ under experimental conditions; powders either white (Li) or green $(\mathrm{Na})$; all air sensitive; ordered structures (Figure 11) from npd at room temperature on deuterides: $R \overline{3} c, Z=6$ (hex setting), $\mathrm{K}_{4} \mathrm{CdCl}_{6}$ type structure; octahedral $\left[\mathrm{TH}_{6}\right]^{4-} 18$-electron complex with T site symmetry $\overline{3}$; surrounded by two $M$ sites in nearly cubic configuration; one D site; lif: $4 \mathrm{M}^{+} \cdot\left[\mathrm{TH}_{6}\right]^{4-} ; \mathrm{T}=\mathrm{Ru}^{\mathrm{II}}, \mathrm{Os}^{\mathrm{II}}$, $\mathrm{d}^{6}$. Interatomic distances are given in Table 8.

\section{$3.12 \mathrm{Na}_{3} \mathrm{RhH}_{6}\left(\mathrm{Li}_{3} \mathrm{RhH}_{6}, \mathrm{Na}_{3} \operatorname{IrH}_{6}, \mathrm{Li}_{3} \operatorname{IrH}_{6}\right)$}

From powder mixtures of binary $\mathrm{MH}$ and $\operatorname{Ir}(\mathrm{Rh})$ at $630-770 \mathrm{~K}$ under hydrogen $;{ }^{73}$ colorless powders, sensitive to air and humidity; $\mathrm{Li}_{3} \mathrm{RhH}_{6}$ forms either during decomposition of $\mathrm{Li}_{3} \mathrm{RhH}_{4}{ }^{74}$ in hydrogen atmosphere or by sintering powder mixtures of $\mathrm{LiH}$ and $\mathrm{Rh}$ under 80 bar hydrogen pressure at $823 \mathrm{~K} ;{ }^{75}$ ordered structures (Figure 12) from npd on deuterides $^{73,75}$ at $295 \mathrm{~K}(\mathrm{Na}-\mathrm{Rh}, \mathrm{Na}-\mathrm{Ir}, \mathrm{Li}-\mathrm{Rh}, \mathrm{Li}-\mathrm{Ir})$ and $11 \mathrm{~K}$ (Na-Rh, Na-Ir); Pnma, $Z=4$; isolated octahedral $\left[\mathrm{TH}_{6}\right]^{3-}$ 18-electron complex with T site symmetry $m$; surrounded by three $\mathrm{M}$ sites in irregular 11-fold coordination; four D sites; no evidence for structural phase transition in $\mathrm{Na}_{3} \mathrm{RhH}_{6}$ and 


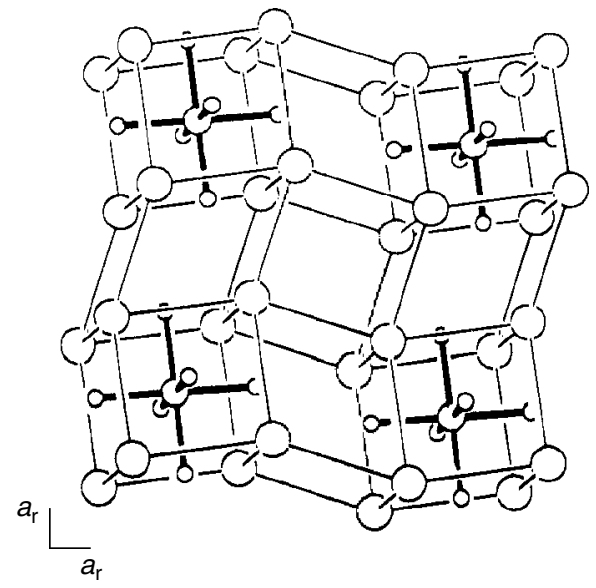

Figure 11 Structure of $\mathrm{Li}_{4} \mathrm{RuD}_{6}$

Table 8 Interatomic distances $(\AA)$ in $\mathrm{Li}_{4} \mathrm{RuD}_{6}$ and analogs

\begin{tabular}{llcl}
\hline & & $\mathrm{T}=\mathrm{Ru}$ & $\mathrm{T}=\mathrm{Os}$ \\
\hline $\mathrm{M}=\mathrm{Li}$ & $\mathrm{T}-\mathrm{D}$ & 1.71 & 1.71 \\
& $\mathrm{~T}-\mathrm{Li}$ & 2.49 & 2.52 \\
& $\mathrm{Li}-\mathrm{D}$ & 2.05 & 2.13 \\
& $\mathrm{Li}-\mathrm{Li}$ & 3.03 & 3.014 \\
& $\mathrm{D}-\mathrm{D}$ & 2.41 & 2.27 \\
$\mathrm{M}=\mathrm{Na}$ & $\mathrm{T}-\mathrm{D}$ & 1.79 & \\
& $\mathrm{~T}-\mathrm{Na}$ & 2.84 & \\
& $\mathrm{Na}-\mathrm{D}$ & 2.35 & \\
& $\mathrm{Na}-\mathrm{Na}$ & 3.40 & \\
& $\mathrm{D}-\mathrm{D}$ & 2.50 & \\
\hline
\end{tabular}

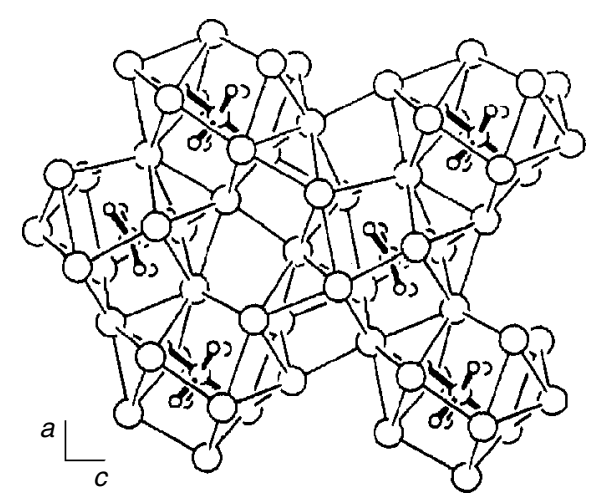

Figure 12 Structure of $\mathrm{Na}_{3} \mathrm{RhD}_{6}$

$\mathrm{Na}_{3} \mathrm{IrH}_{6}$ down to $11 \mathrm{~K}$ and up to $670 \mathrm{~K}$; lif: $3 \mathrm{M}^{+} \cdot\left[\mathrm{TH}_{6}\right]^{3-}$; $\mathrm{T}=\mathrm{Ir}^{\mathrm{III}}, \mathrm{Rh}^{\mathrm{III}} ; \mathrm{d}^{6}$. Interatomic distances are given in Table 9.

\subsection{3 $\mathrm{Li}_{3} \mathrm{RhH}_{4}$}

From $\mathrm{LiH}$ and $\mathrm{Rh}$ powder at $870 \mathrm{~K}$ under hydrogen; ${ }^{74}$ powder of metallic appearance; oxygen and moisture sensitive;
Table 9 Interatomic distances $(\AA)$ in $\mathrm{Na}_{3} \mathrm{RhD}_{6}$ and analogs

\begin{tabular}{llcc}
\hline & & $\mathrm{T}=\mathrm{Rh}$ & $\mathrm{T}=\mathrm{Ir}$ \\
\hline $\mathrm{M}=\mathrm{Li}$ & $\mathrm{T}-\mathrm{D}$ & $1.56-1.64$ & $1.61-1.70$ \\
& $\mathrm{~T}-\mathrm{Li}$ & 2.66 & 2.72 \\
& $\mathrm{Li}-\mathrm{D}$ & 1.81 & 1.88 \\
& $\mathrm{Li}-\mathrm{Li}$ & 2.59 & 2.59 \\
& $\mathrm{D}-\mathrm{D}$ & 2.07 & 2.21 \\
$\mathrm{M}=\mathrm{Na}$ & $\mathrm{T}-\mathrm{D}$ & $1.63-1.68$ & $1.64-1.70$ \\
& $\mathrm{~T}-\mathrm{Na}$ & 2.98 & 2.94 \\
& $\mathrm{Na}-\mathrm{D}$ & 2.17 & 2.08 \\
& $\mathrm{Na}-\mathrm{Na}$ & 3.22 & 3.18 \\
& $\mathrm{D}-\mathrm{D}$ & 2.28 & 2.26 \\
\hline
\end{tabular}

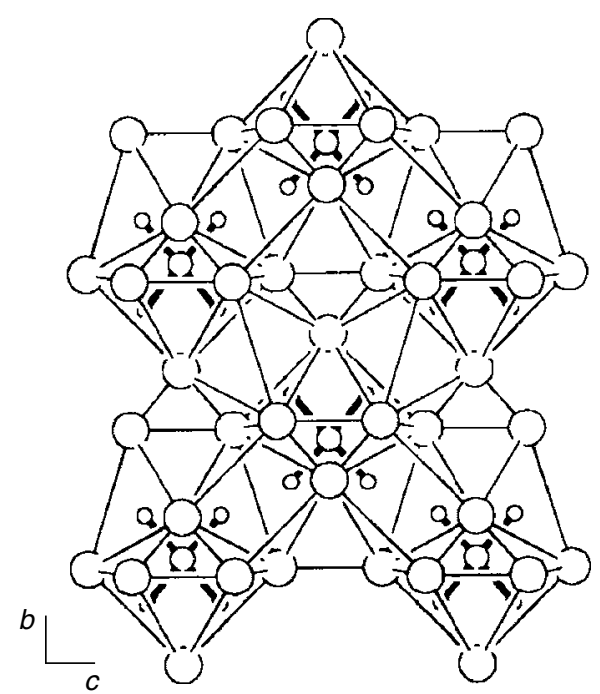

Figure 13 Structure of $\mathrm{Li}_{3} \mathrm{RhD}_{4}$

corresponds to previously reported ' $\mathrm{Li}_{4} \mathrm{RhH}_{5}$ ' as characterized by X-ray diffraction on single crystals; ${ }^{76}$ decomposes at high temperature under 80 bar hydrogen pressure into $\mathrm{Li}_{3} \mathrm{RhH}_{6}$ (XII); ordered structure (Figure 13) by npd on deuteride: Cmcm; $\mathrm{Z}=4$; planar $\left[\mathrm{RhH}_{4}\right]^{3-}$ 16-electron complexes with T site symmetry $m 2 m$; surrounded by two Li sites in irregular 11-fold coordination; two D sites; lif: $3 \mathrm{Li}^{+} \cdot\left[\mathrm{RhH}_{4}\right]^{3-} ; \mathrm{Rh}^{\mathrm{I}}$, $\mathrm{d}^{8}$.

Distances $(\AA): \mathrm{Rh}-\mathrm{D}=1.79(2 \times), 1.75(2 \times) ; \mathrm{Rh}-\mathrm{Li}=$ 2.31; $\quad \mathrm{Rh}-\mathrm{Rh}=3.87(2 \times) ; \quad \mathrm{Li}-\mathrm{Li}=2.35 ; \quad \mathrm{Li}-\mathrm{D}=2.15$; $\mathrm{D}-\mathrm{D}=2.21$.

\subsection{4 $\operatorname{MgRhH}_{1-x}$}

From binary alloy $\mathrm{MgRh}$ (CsCl-type structure) at $748 \mathrm{~K}$ and 130 bar hydrogen pressure ${ }^{77}$ powder of gray color and metallic appearance; partially disordered structure (Figure 14) by in situ npd at 39 bar, 2.5 bar and $10^{-2}$ mbar deuterium pressure: $P 4 / \mathrm{mmm}, Z=4$; one $\mathrm{Rh}$ and two $\mathrm{Mg}$ sites forming a tetragonal distorted CsCl-type structure with doubled $a$ parameter $(2 c / a=1.012$ at 39 bar $)$; one D site connecting 


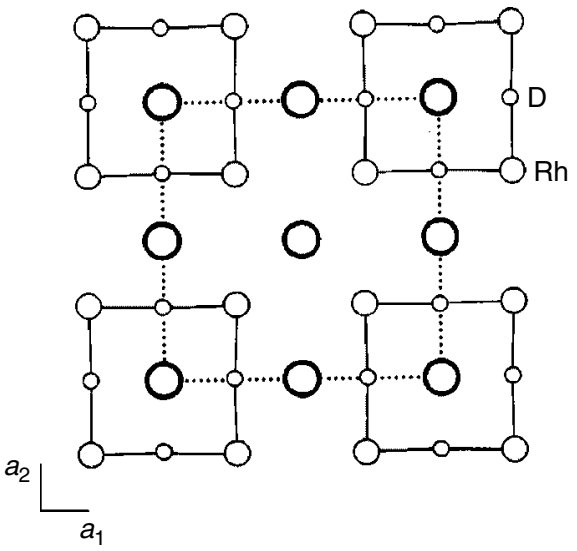

Figure 14 Structure of $\mathrm{MgRhD}_{1-x}$

$\mathrm{Rh}$ into cyclic tetramer $\mathrm{Rh}_{4} \mathrm{D}_{4}$ having 4/mmm symmetry with nearly linear $\mathrm{Rh}-\mathrm{D}-\mathrm{Rh}$ and rectangular $\mathrm{D}-\mathrm{Rh}-\mathrm{D}$ bonds; occupancy of D site increasing as a function of D pressure (94\% at 39 bar, $86 \%$ at 2.5 bar, $61 \%$ at $10^{-2}$ mbar), metal substructure resembles $\mathrm{CaPdH}_{2}(\mathrm{X})$; likely phase limit near stoichiometric composition $\mathrm{MgRhH}$; lif (border case) at that composition: $4 \mathrm{Mg}^{2+} \cdot\left[\mathrm{Rh}_{4} \mathrm{H}_{4}\right]^{8-}$; X-ray evidence for cubic $\operatorname{MgIrH}_{x}$ phase $(a=3.23 \AA)$ at high pressure with CsCl-type metal substructure.

Distances $(\AA)$ at 39 bar $\left(\mathrm{MgRhD}_{0.94}\right)$ : Rh-D $(2 \times)=1.71$; $\mathrm{Mg}-\mathrm{Rh}=2.66 ; \mathrm{Mg}-\mathrm{D}=2.23 ; \mathrm{Rh}-\mathrm{Rh}=2.98 ; \mathrm{Mg}-\mathrm{Mg}=$ $3.20 ; \mathrm{D}-\mathrm{D}=2.36$.

\subsection{5 $\mathrm{Mg}_{3} \mathrm{RuH}_{3}$}

From elements by sintering at $883 \mathrm{~K}$ under 9 bar hydrogen pressure $;^{78}$ dark gray powder, stable in air; partially disordered structure (Figure 15) by npd on deuteride: $P 4_{2} / \mathrm{mnm}$, $Z=4$; $\mathrm{Ru}$ at center of orientationally disordered ts- $\mathrm{RuH}_{3}$ units ( $m .2 m$ site symmetry) containing two D sites, one ordered and the other disordered (split atom position with $\mathrm{D}-\mathrm{D}=0.87 \AA$ ); surrounded by two $\mathrm{Mg}$ sites forming an eightfold bicapped prismatic coordination; joined to dimers of $m . m m$ site symmetry by possible $\mathrm{Ru}-\mathrm{Ru}$ bond; lif: $3 \mathrm{Mg}^{2+} \cdot\left[\mathrm{RuH}_{3}\right]^{6-}$ (monomer without $\mathrm{Ru}-\mathrm{Ru}$ bond, 17 electrons/ $\mathrm{Ru}$ ); $6 \mathrm{Mg}^{2+} \cdot\left[\mathrm{Ru}_{2} \mathrm{H}_{6}\right]^{12-}$ (dimer with $\mathrm{Ru}-\mathrm{Ru}$ bond, 18 electrons/Ru); $\mathrm{Ru}-\mathrm{Ru}$ bond formation not supported by extended Hückel calculations. ${ }^{\mathbf{1 2}}$

Distances $(\AA)$ : $\mathrm{Ru}-\mathrm{D}=1.71$ (split atom), $1.71(2 \times)$; $\mathrm{Mg}-\mathrm{Ru}=2.73 ; \mathrm{Ru}-\mathrm{Ru}=3.31 ; \mathrm{Mg}-\mathrm{D}=2.01 ; \mathrm{Mg}-\mathrm{Mg}=$ 3.03; D-D $=0.87$ (split atom), 2.52.

\subsection{6 $\mathrm{SrMg}_{2} \mathrm{FeH}_{8}\left(\mathrm{BaMg}_{2} \mathrm{FeH}_{8}, \mathrm{EuMg}_{2} \mathrm{FeH}_{8}\right)$}

From binary alloys $\mathrm{SrMg}_{2.2}\left(\mathrm{BaMg}_{2}, \mathrm{EuMg}_{2}\right)$ and $\mathrm{Fe}$ powder at $763-783 \mathrm{~K}$ under 140 bar hydrogen pressure; ${ }^{\mathbf{6 6 , 7 9}}$ green $(\mathrm{Sr})$ or brownish $(\mathrm{Ba})$ powder; sensitive to air; ordered structure (Figure 16) by npd on deuterides of $\mathrm{Sr}$ and $\mathrm{Ba}$
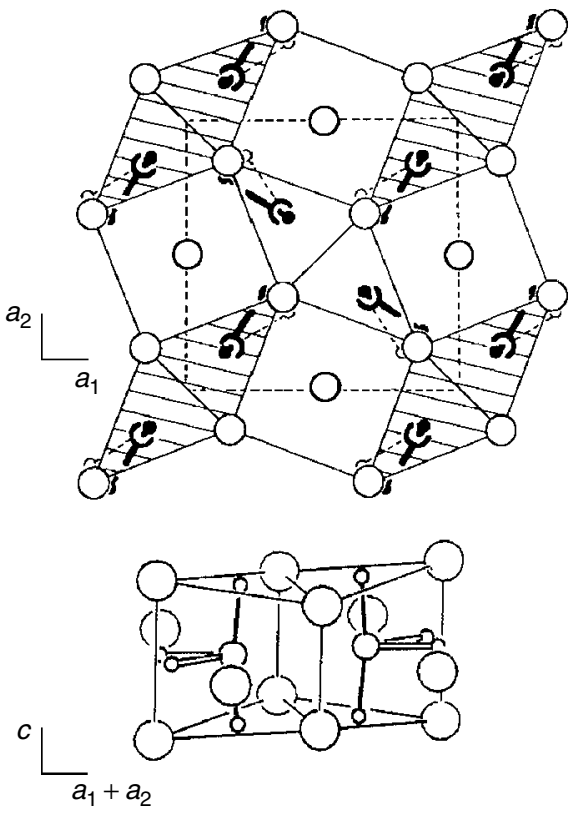

Figure 15 Structure of $\mathrm{Mg}_{3} \mathrm{RuD}_{3}$

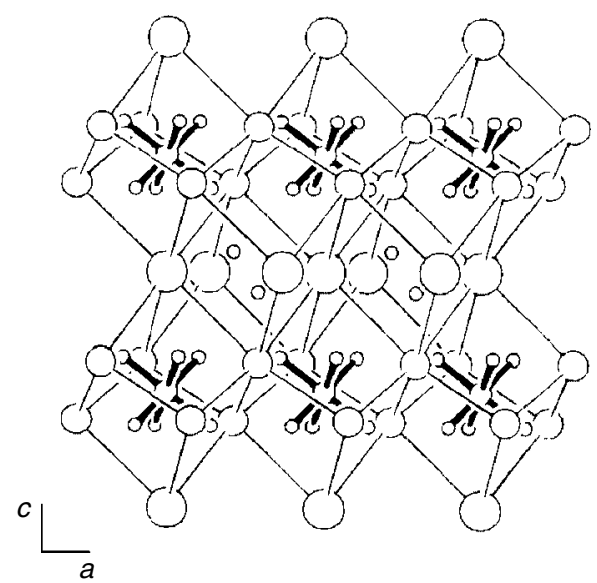

Figure 16 Structure of $\mathrm{SrMg}_{2} \mathrm{FeD}_{8}$

compounds: $P \overline{3} m 1, Z=1$; octahedral $\left[\mathrm{FeH}_{6}\right]^{4-}$ 18-electron units having T site symmetry $\overline{3} m$, surrounded by eight $\mathrm{M}^{2+}$ in nearly cubic configuration; one $\mathrm{Mg}$ and one $\mathrm{Sr}(\mathrm{Ba})$ site; two $\mathrm{D}$ sites, of which the twofold is tetrahedrally coordinated by $\mathrm{M}^{2+}$ with one short $\mathrm{Mg}-\mathrm{D}$ and three long $\mathrm{Sr}(\mathrm{Ba})-\mathrm{D}$ bonds; lif: $\mathrm{M}^{2+} \cdot 2 \mathrm{Mg}^{2+} \cdot\left[\mathrm{FeH}_{6}\right]^{4-} \cdot 2 \mathrm{H}^{-} ; \mathrm{Fe}^{\mathrm{II}}, \mathrm{d}^{6} . \mathrm{Ru}$ analog $\mathrm{BaMg}_{2} \mathrm{RuD}_{8}$ (XXXVIII) crystallizes with tetragonal symmetry. Interatomic distances are given in Table 10.

\section{$3.17 \mathrm{Mg}_{6} \mathrm{Co}_{2} \mathrm{H}_{11}$}

From elements by sintering at 753-773 K under 40-50 bar hydrogen pressure, ${ }^{80}$ black powder, stable in air; nonmetallic; pressure-composition isotherms show two plateaus of 
Table 10 Interatomic distances $(\AA)$ in $\mathrm{SrMg}_{2} \mathrm{FeD}_{8}$ and barium analog

\begin{tabular}{lcc}
\hline & $\mathrm{M}=\mathrm{Sr}$ & $\mathrm{M}=\mathrm{Ba}$ \\
\hline Fe-D & 1.58 & 1.58 \\
Fe-Mg & 2.72 & 2.75 \\
Fe-M & 3.28 & 3.46 \\
Mg-D & 2.20 & 2.22 \\
Mg-D & 1.92 & 1.92 \\
M-D & 2.65 & 2.81 \\
M-D & 2.66 & 2.74 \\
Mg-Mg & 3.06 & 3.08 \\
Mg-M & 3.60 & 3.75 \\
D-D & 2.19 & 2.18 \\
\hline
\end{tabular}

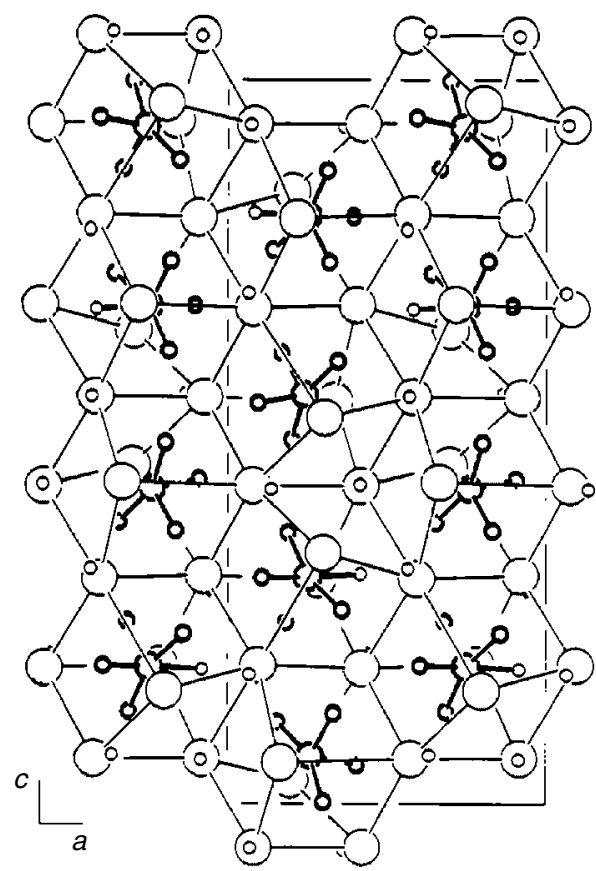

Figure 17 Structure of $\mathrm{Mg}_{6} \mathrm{Co}_{2} \mathrm{D}_{11}$

which that at low pressure is attributed to decomposition of $\mathrm{Mg}_{6} \mathrm{Co}_{2} \mathrm{H}_{11}\left(\Delta H=79 \mathrm{~kJ} \mathrm{~mol}^{-1} \mathrm{H}_{2}\right)$ and that at high pressure to decomposition of tetragonal $\mathrm{Mg}_{2} \mathrm{CoH}_{5}\left(\Delta H=79 \mathrm{~kJ} \mathrm{~mol}^{-1}\right.$ $\left.\mathrm{H}_{2}\right){ }^{\mathbf{8 1}}$ partially disordered structure (Figure 17) from joint synchrotron X-ray and high-resolution npd: ${ }^{80}$ Pnma, $Z=8$, 63 positional parameters; two eightfold Co sites, one (Col) forming an ordered sad-[ $\left[\mathrm{CoH}_{4}\right]^{5-} 18$-electron complex of symmetry 1 , and the other $(\mathrm{Co} 2)$ a partially disordered spy configuration with one half-occupied ligand; interpreted as a 1:1 mixture of spy- $\left[\mathrm{CoH}_{5}\right]^{4-}$ and sad- $\left[\mathrm{CoH}_{4}\right]^{5-} 18$-electron complexes; surrounded by eight $\mathrm{Mg}$ sites in distorted cubic configurations; $14 \mathrm{D}$ sites, of which five are surrounded by $\mathrm{Mg}$ in tri and sad configurations; metal substructure derives from trigonal $\mathrm{SrMg}_{2} \mathrm{FeH}_{8}$ (XVI) by orthorhombic distortion; short $\mathrm{Mg}-\mathrm{Mg}$ bonds; lif: $2 \mathrm{Mg}_{6} \mathrm{Co}_{2} \mathrm{H}_{11}=$ $12 \mathrm{Mg}^{2+} \cdot\left[\mathrm{CoH}_{5}\right]_{\mathrm{av}}{ }^{4-} \cdot 2\left[\mathrm{CoH}_{4}\right]^{5-} \cdot\left[\mathrm{CoH}_{4}\right]_{\mathrm{av}}{ }^{5-} \cdot 5 \mathrm{H}^{-} ; \quad$ mono- clinic distorted $\mathrm{Ir}$ analog $\mathrm{Mg}_{6} \mathrm{Ir}_{2} \mathrm{H}_{11}$ (XLV) has different $\mathrm{T}-\mathrm{H}$ complex ordering; other hydrides with similar metal substructure: $\mathrm{Mg}_{3} \mathrm{RuH}_{6}$ (XXV), $\mathrm{Mg}_{3} \mathrm{ReH}_{7}$ (XXIII), and $\mathrm{LiMg}_{2} \mathrm{RuH}_{7}$ (XXVII).

Distances $(\AA)$ : Co1-D = 1.52-1.57, Co2-D = 1.51-1.62; $\mathrm{Co}-\mathrm{Co}=4.58 ; \mathrm{Co}-\mathrm{Mg}=2.52 ; \mathrm{Mg}-\mathrm{Mg}=2.78 ; \mathrm{Mg}-\mathrm{D}=$ $1.94 ; \mathrm{Mg}^{-} \mathrm{D}^{-}=1.82 ; \mathrm{D}-\mathrm{D}=2.09$.

\subsection{8 $\mathrm{Mg}_{2} \mathrm{RuH}_{4}$}

From the elements, by sintering at $723 \mathrm{~K}$ under 20 bar, or at $1050 \mathrm{~K}$ under 160 bar hydrogen pressure; ${ }^{\mathbf{8 2}}$ powder of reddish-brown to dark-red color, sensitive to moisture and air; ordered structure (Figure 18) from npd on deuteride: Cmcm, $\mathrm{Z}=4$; sad-[ $\left[\mathrm{RuH}_{4}\right]^{4-}$ 16-electron complex with T site symmetry $m 2 m$, derives from the oct-[RuH$]^{4-}$ complex in $\mathrm{Mg}_{2} \mathrm{RuH}_{6}$ by removal of two cis D ligands; two D sites; surrounded by $\mathrm{Mg}$ in eightfold distorted cubic configuration, neighboring cubes share faces; possible $\mathrm{Ru}-\mathrm{Ru}$ interactions across these faces ( $\mathrm{Ru}-\mathrm{Ru}=3.24 \AA$ ) could lead to polyanionic $\left[\mathrm{RuH}_{4}\right]_{n}{ }^{4 n-}$ zig-zag chains running along $c$; magnetic measurements consistent with $\mathrm{d}^{8}$ low-spin configuration; lif: $2 \mathrm{Mg}^{2+} \cdot\left[\mathrm{RuH}_{4}\right]^{4-}$ (monomer); $\mathrm{Ru}^{0}, \mathrm{~d}^{8} ; 2 n \mathrm{Mg}^{2+} \cdot\left[\mathrm{Ru}_{n} \mathrm{H}_{4 n}\right]^{4 n-}$ (polymer); Ru-Ru bonds not supported by extended Hückel calculations. ${ }^{\mathbf{1 2}}$

Distances $(\AA)$ and angles: $\mathrm{Ru}-\mathrm{D}=1.67(2 \times), 1.68(2 \times)$; $\mathrm{D}-\mathrm{Ru}-\mathrm{D}=84.2^{\circ}, 93.6^{\circ}$ (cis), $170.3^{\circ}$ (trans); $\mathrm{Ru}-\mathrm{Mg}=2.69$; $\mathrm{Ru}-\mathrm{Ru}=3.24(2 \times) ; \mathrm{Mg}-\mathrm{D}=2.10 ; \mathrm{Mg}-\mathrm{Mg}=2.93 ; \mathrm{D}-\mathrm{D}=$ 2.23 .

\subsection{9 $\mathrm{CaMgNiH}_{4}\left(\mathrm{SrMgNiH}_{4}, \mathrm{YbMgNiH}_{4}, \mathrm{EuMgNiH}_{4}\right)$}

From mixtures of arc-melted binary alloys $\left(\mathrm{CaNi}_{2}, \mathrm{CaMg}_{2}\right.$, $\mathrm{SrNi}, \mathrm{YbNi}, \mathrm{EuNi}$ ) or $\mathrm{Mg}$ sintered at $723-763 \mathrm{~K}$ under

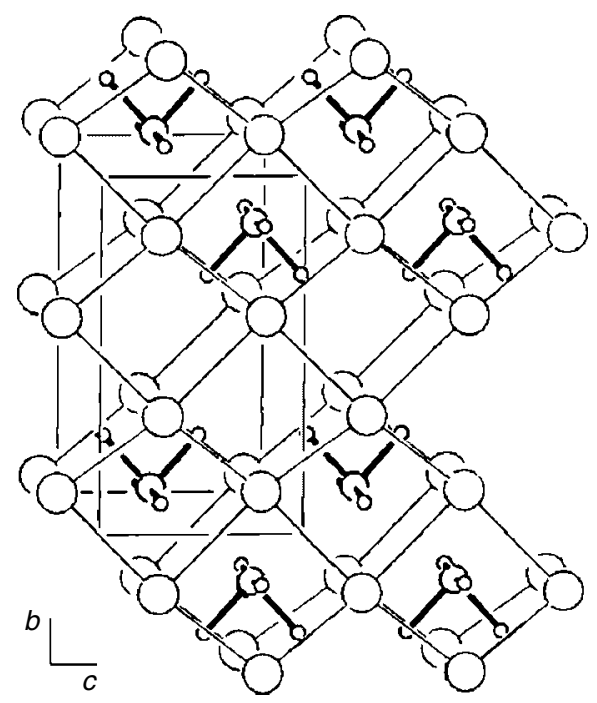

Figure 18 Structure of $\mathrm{Mg}_{2} \mathrm{RuD}_{4}$ 


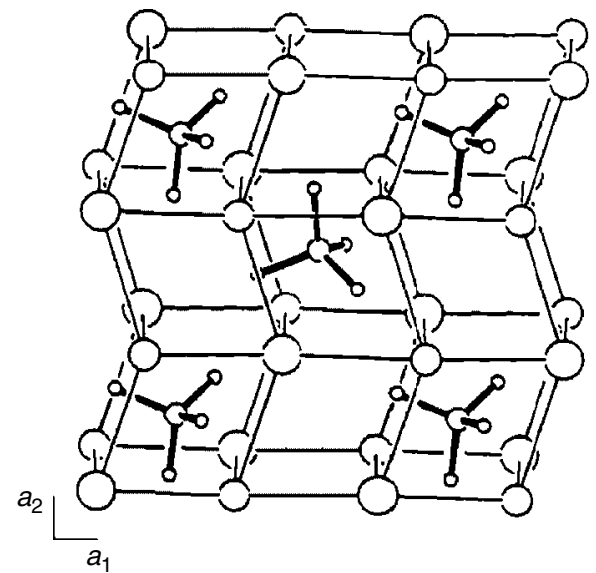

Figure 19 Structure of $\mathrm{CaMgNiD}_{4}$

Table 11 Interatomic distances (A) in $\mathrm{CaMgNiD}_{4}$ and analogs

\begin{tabular}{lccc}
\hline & $\mathrm{M}=\mathrm{Ca}$ & $\mathrm{M}=\mathrm{Sr}$ & $\mathrm{M}=\mathrm{Yb}$ \\
\hline $\mathrm{Ni}-\mathrm{D}$ & $1.59-1.61$ & $1.54-1.64$ & $1.57-1.62$ \\
$\mathrm{Ni}-\mathrm{Mg}$ & 2.54 & 2.55 & 2.53 \\
$\mathrm{Ni}-\mathrm{M}$ & 3.03 & 3.21 & 3.03 \\
$\mathrm{Mg}-\mathrm{D}$ & 2.08 & 2.15 & 2.09 \\
$\mathrm{M}-\mathrm{D}$ & 2.45 & 2.53 & 2.43 \\
$\mathrm{Mg}-\mathrm{Mg}$ & 4.18 & 4.24 & 4.15 \\
$\mathrm{Mg}-\mathrm{M}$ & 3.48 & 3.54 & 3.50 \\
$\mathrm{D}-\mathrm{D}$ & 2.59 & 2.54 & 2.60 \\
$\mathrm{D}-\mathrm{Ni}-\mathrm{D}$ & $108^{\circ}, 111^{\circ}$ & $108^{\circ}, 111^{\circ}$ & $109^{\circ}, 110^{\circ}$ \\
\hline
\end{tabular}

130-140 bar hydrogen pressure ${ }^{\mathbf{8 3}}$ greenish (Ca, Sr) or black (Yb) powders; translucent single crystals of $\mathrm{CaMgNiH}_{4}$ having yellow-brownish color obtained by sintering binary hydrides with $\mathrm{LiH}$ flux in Ni crucible at $737 \mathrm{~K}$ under 95 bar hydrogen pressure ${ }^{84}$ desorption enthalpy $\Delta H=129$ (3) (Ca), $111(3)(\mathrm{Yb}) \mathrm{kJ} \mathrm{mol}^{-1} \mathrm{H}_{2}$; ordered structures (Figure 19) from npd on deuterides ( $\mathrm{Ca}, \mathrm{Sr}$, and $\left.\mathrm{Yb}^{\mathbf{8 3}}\right): P 2_{1} 3, Z=4$; tetrahedral $\left[\mathrm{NiH}_{4}\right]^{4-}$ 18-electron complex having $\mathrm{T}$ site symmetry 3 , surrounded by one $\mathrm{Ca}(\mathrm{Sr}, \mathrm{Yb})$ site, and one $\mathrm{Mg}$ site in ordered distorted cubic configuration; two D sites; derived from $\mathrm{Mg}_{2} \mathrm{NiD}_{4}(\mathrm{~V})$ by partial substitution of $\mathrm{Mg}$; lif: $\mathrm{M}^{2+} \cdot \mathrm{Mg}^{2+} \cdot\left[\mathrm{NiH}_{4}\right]^{4-} ; \mathrm{Ni}^{0}, \mathrm{~d}^{10} ; \mathrm{X}$-ray evidence for isostructural $\mathrm{EuMgNiH}_{4} \cdot{ }^{\mathbf{8 3}}$ Interatomic distances are given in Table 11.

\section{$3.20 \mathrm{Ca}_{4} \mathrm{Mg}_{4} \mathrm{Fe}_{3} \mathrm{H}_{22}\left(\mathrm{Yb}_{4} \mathrm{Mg}_{4} \mathrm{Fe}_{3} \mathrm{H}_{22}\right)$}

From mixtures of arc-melted binary alloys (CaMg, $\mathrm{YbMg}$ ) and $\mathrm{Fe}$ powder, by adding $\mathrm{LiH}$ as a flux, at about $773-793$ $\mathrm{K}$ under $100-150$ bar hydrogen pressure, ${ }^{85} \mathrm{Ca}_{4} \mathrm{Mg}_{4} \mathrm{Fe}_{3} \mathrm{H}_{22}$ : brownish powder containing brownish translucent single crystals; $\mathrm{Yb}_{4} \mathrm{Mg}_{4} \mathrm{Fe}_{3} \mathrm{H}_{22}$ : black powder; both stable in air for several weeks; desorption enthalpies $\Delta H=122(4)$ (Ca), 137(3) $(\mathrm{Yb}) \mathrm{kJ} \mathrm{mol}^{-1} \mathrm{H}_{2}$; decomposition of $\mathrm{Ca}$ compound

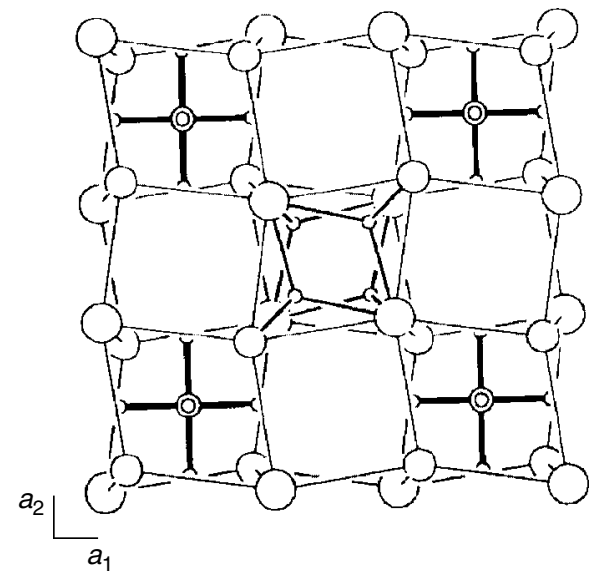

Figure 20 Structure of $\mathrm{Ca}_{4} \mathrm{Mg}_{4} \mathrm{Fe}_{3} \mathrm{D}_{22}$

Table 12 Interatomic distances $(\AA)$ in $\mathrm{Ca}_{4} \mathrm{Mg}_{4} \mathrm{Fe}_{3} \mathrm{D}_{22}$ and ytterbium analog

\begin{tabular}{lcc}
\hline & $\mathrm{M}=\mathrm{Ca}$ & $\mathrm{M}=\mathrm{Yb}$ \\
\hline $\mathrm{Fe}-\mathrm{D}$ & $1.56-1.58$ & $1.55-1.59$ \\
$\mathrm{Fe}-\mathrm{Mg}$ & 2.79 & 2.78 \\
$\mathrm{Fe}-\mathrm{M}$ & 3.09 & 3.06 \\
$\mathrm{Mg}-\mathrm{D}$ & 2.07 & 2.02 \\
$\mathrm{Mg}-\mathrm{D}^{-}$ & 1.81 & 1.91 \\
$\mathrm{M}-\mathrm{D}$ & 2.43 & 2.41 \\
$\mathrm{M}-\mathrm{D}^{-}$ & 2.39 & 2.41 \\
$\mathrm{Mg}-\mathrm{Mg}$ & 4.08 & 3.98 \\
$\mathrm{Mg}-\mathrm{M}$ & 3.39 & 3.40 \\
$\mathrm{D}-\mathrm{D}$ & 2.22 & 2.22 \\
\hline
\end{tabular}

shows two plateaus: formation of $\mathrm{Ca}_{2} \mathrm{FeH}_{6}, \mathrm{Mg}, \mathrm{Fe}$, and $\mathrm{H}_{2}$ (upper plateau), and of $\mathrm{CaH}_{2}, \mathrm{Fe}$, and $\mathrm{H}_{2}$ (lower plateau); decomposition of $\mathrm{Yb}$ compound shows one plateau: formation of $\mathrm{YbH}_{2}, \mathrm{Mg}, \mathrm{Fe}$, and $\mathrm{H}_{2}$; ordered structures (Figure 20) from single-crystal $\mathrm{X}$-ray $\left(\mathrm{Ca}_{4} \mathrm{Mg}_{4} \mathrm{Fe}_{3} \mathrm{H}_{22}\right)$ and npd on deuterides; $P \overline{4} 3 m, Z=1$; octahedral $\left[\mathrm{FeH}_{6}\right]^{4-}$ 18-electron complexes with T site symmetry $\overline{4} 2 . m$, surrounded by one $\mathrm{M}$ and one $\mathrm{Mg}$ site in deformed cubic configuration; three D sites, of which the fourfold is tetrahedrally surrounded by one $\mathrm{Mg}^{2+}$ and three $\mathrm{M}^{2+}$; derived from $\mathrm{Mg}_{2} \mathrm{FeH}_{6}$ (II) by partial substitution of $\mathrm{Mg}$ by $\mathrm{Ca}$, and of every fourth $\left[\mathrm{FeH}_{6}\right]^{4-}$ by $4 \mathrm{H}^{-}$; lif: $4 \mathrm{M}^{2+} .4 \mathrm{Mg}^{2+} \cdot 3\left[\mathrm{FeH}_{6}\right]^{4-} .4 \mathrm{H}^{-} ; \mathrm{Fe}^{\mathrm{II}}, \mathrm{d}^{6}$. Interatomic distances are given in Table 12.

\subsection{1 $\mathrm{Ba}_{2} \mathrm{PtH}_{6}\left(\mathrm{Sr}_{2} \mathrm{PtH}_{6}\right)$}

From $\mathrm{BaH}_{2}\left(\mathrm{SrH}_{2}\right)$ and Pt powder in hydrogen; ${ }^{86}$ ordered $\mathrm{Ba}_{2} \mathrm{CuF}_{6}$ type structure (Figure 21) from npd on deuteride: Cmca, $Z=4$; planar $\left[\mathrm{PtD}_{4}\right]^{2-}$ 16-electron complexes with $\mathrm{T}$ site symmetry $2 / \mathrm{m}$, surrounded by $\mathrm{M}$ in 10 -fold bicapped cubic configuration; three $\mathrm{D}$ sites, of which one eightfold is tetrahedrally surrounded by $\mathrm{M}^{2+}$; lif: $2 \mathrm{M}^{2+} \cdot\left[\mathrm{PtH}_{4}\right]^{2-} \cdot 2 \mathrm{H}^{-}$; $\mathrm{Pt}^{\mathrm{II}}, \mathrm{d}^{8}$. 


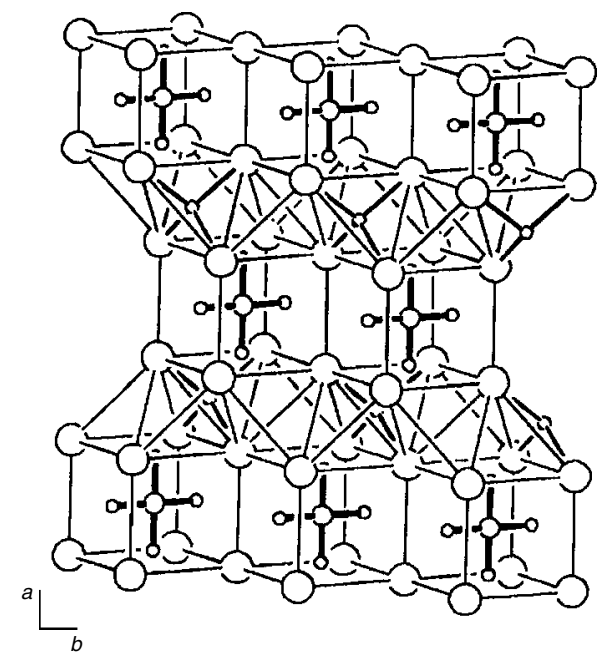

Figure 21 Structure of $\mathrm{Ba}_{2} \mathrm{PtD}_{6}$

Distances $(\AA)$ : Pt-D = 1.66(2×) and $1.67(2 \times)$; Pt-Ba $=$ 3.63; $\mathrm{Pt}-\mathrm{Pt}=4.24 ; \mathrm{Ba}-\mathrm{Ba}=4.09 ; \mathrm{Ba}-\mathrm{D}=2.97 ; \mathrm{Ba}-\mathrm{D}^{-}=$ 2.62; D-D $=2.36$.

\subsection{2 $\mathrm{LiSr}_{2} \mathrm{PdH}_{5}$}

From reaction of arc-melted ternary alloy $\mathrm{Li}_{1.5} \mathrm{Sr}_{2} \mathrm{Pd}$ at $793-803 \mathrm{~K}$ under 155 bar hydrogen pressure, ${ }^{87}$ darkgreen powder, sensitive to air; partially disordered structure (Figure 22) from npd on deuteride at $14 \mathrm{~K}: P 4 / \mathrm{mmm}, Z=1$; metals form ordered superstructure of $\mathrm{CsCl}$; contains ordered $\mathrm{SrLiD}_{3}$ type slabs having inverse perovskite-type structure; Pd surrounded by three D, on average, in partially disordered octahedral configuration with four half occupied equatorial $\mathrm{D}$ sites forming linear Pd-D-Pd bridges similar to those in $\mathrm{CaPdH}_{2}(\mathrm{X})$, and two fully occupied axial sites belonging to the $\mathrm{SrLiD}_{3}$ type slabs; both have large displacement amplitudes; three D sites, of which a twofold is octahedral surrounded

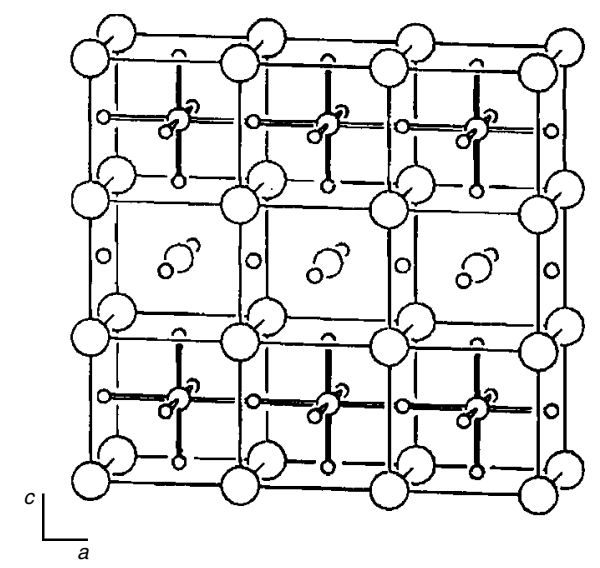

Figure 22 Structure of $\mathrm{LiSr}_{2} \mathrm{PdD}_{5}$ by four $\mathrm{Sr}$ and two $\mathrm{Li}$; lif: $\mathrm{Li}^{+} \cdot 2 \mathrm{Sr}^{2+} \cdot\left[\mathrm{PdH}_{3}\right]_{\mathrm{av}}{ }^{3-} \cdot 2 \mathrm{H}^{-} ; \mathrm{Pd}^{0}$, $\mathrm{d}^{10}$ (average).

Distances $(\AA): \mathrm{Pd}-\mathrm{D}=1.69(2 \times), 1.95$ (4×, disordered); $\mathrm{Pd}-\mathrm{Sr}=3.34 ; \mathrm{Pd}-\mathrm{Li}=3.70 ; \mathrm{Pd}-\mathrm{Pd}=3.90 ; \mathrm{Li}-\mathrm{D}=2.01$; $\mathrm{Li}-\mathrm{D}^{-}=1.95 ; \mathrm{Li}-\mathrm{Sr}=3.30 ; \mathrm{Sr}-\mathrm{D}=2.71 ; \mathrm{Sr}-\mathrm{D}^{-}=2.66$; $\mathrm{D}-\mathrm{D}=2.58$.

\subsection{3 $\mathrm{Mg}_{3} \mathrm{ReH}_{7}\left(\mathrm{Mg}_{3} \mathrm{MnH}_{7}\right)$}

From elements by sintering powder mixtures at 783-793 K under 120 bar hydrogen pressure; ${ }^{\mathbf{8 8}}$ gray powder; sensitive to air; orange-red $\mathrm{Mn}$ analog $\mathrm{Mg}_{3} \mathrm{MnH}_{7}$ from powder mixtures of $\mathrm{MgH}_{2}$ and $\mathrm{Mn}$ at $20 \mathrm{kbar}$ pressure and $1073 \mathrm{~K}$ in multianvil device; ${ }^{\mathbf{8 9}}$ ordered structures (Figure 23 ) by npd on deuterides: $P 6_{3} / m m c, Z=2$; octahedral $\left[\mathrm{TH}_{6}\right]^{5-}$ 18-electron complexes with $\mathrm{T}$ site symmetry $\overline{3} \mathrm{~m}$, surrounded by two $\mathrm{Mg}$ sites in eightfold distorted cubic configuration similar to that of $\mathrm{SrMg}_{2} \mathrm{FeH}_{8}$ (XVI); two D sites, of which the twofold is surrounded by $\mathrm{Mg}$ in trigonal bipyramidal configuration with two short collinear and three long triangular $\mathrm{Mg}-\mathrm{D}$ bonds; lif: $3 \mathrm{Mg}^{2+} \cdot\left[\mathrm{TH}_{6}\right]^{5-} \cdot \mathrm{H}^{-} ; \mathrm{T}^{\mathrm{I}}, \mathrm{d}^{6}$; electronic band structure calculation on $\mathrm{Mn}$ compound. ${ }^{\mathbf{9 0}}$ Interatomic distances are given in Table 13.

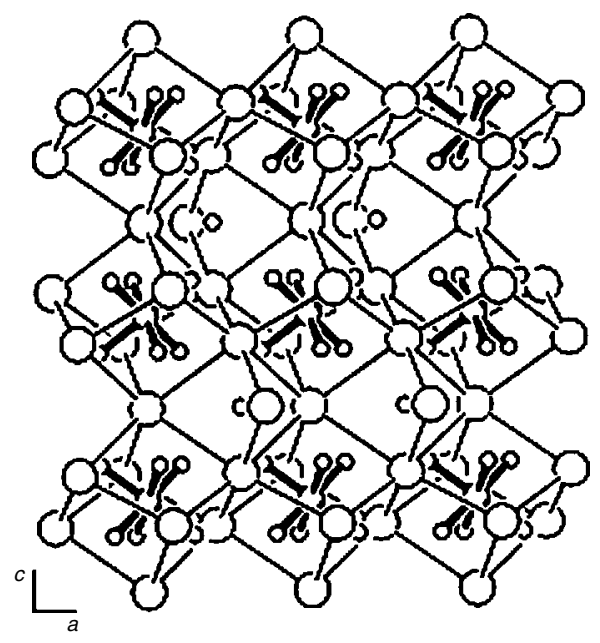

Figure 23 Structure of $\mathrm{Mg}_{3} \operatorname{ReD}_{7}$

Table 13 Interatomic distances $(\AA)$ in $\mathrm{Mg}_{3} \mathrm{ReD}_{7}$ and manganese analog

\begin{tabular}{lcc}
\hline & $\mathrm{T}=\mathrm{Mn}$ & $\mathrm{T}=\mathrm{Re}$ \\
\hline $\mathrm{T}-\mathrm{D}$ & 1.63 & 1.72 \\
$\mathrm{~T}-\mathrm{Mg}$ & 2.56 & 2.64 \\
$\mathrm{~T}-\mathrm{T}$ & 4.70 & 4.85 \\
$\mathrm{Mg}-\mathrm{D}$ & 2.08 & 2.14 \\
Mg-D & 1.87 & 1.90 \\
Mg-Mg & 3.05 & 3.17 \\
D-D & 2.29 & 2.41 \\
\hline
\end{tabular}




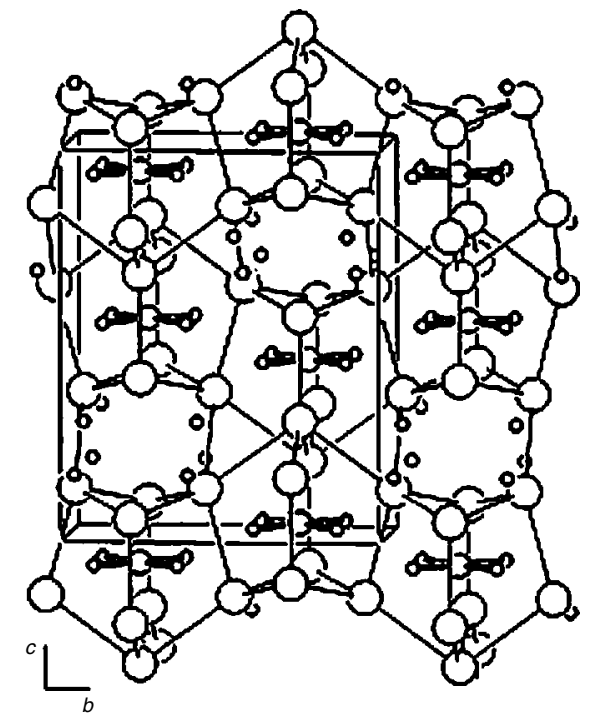

Figure 24 Structure of $\mathrm{Mg}_{4} \mathrm{IrD}_{5}$

\section{$3.24 \mathrm{Mg}_{4} \mathrm{IrH}_{5}$}

From elements by sintering powder mixtures at $783 \mathrm{~K}$ and 41 bar hydrogen pressure; ${ }^{91}$ dark gray powder, stable in air; presumably metallic; partially disordered structure (Figure 24) from npd on deuteride at room temperature and $9 \mathrm{~K}$ : Imma, $Z=4$; Ir surrounded by three $\mathrm{D}$ atoms, on the average, in disordered planar configuration with $75 \%$ occupancy (average $\mathrm{T}$ site symmetry $\mathrm{mm} 2$ ); surrounded by three $\mathrm{Mg}$ sites in irregular ninefold coordination; two D sites, of which the eightfold is coordinated by $\mathrm{Mg}$ in saddlelike configuration; short $\mathrm{Mg}-\mathrm{Mg}$ bonds along backbone of $\left[\mathrm{Mg}_{9} \mathrm{IrD}_{3}\right]$ units; no apparent homogeneity range; lif (border case): $4 \mathrm{Mg}^{2+} \cdot\left[\mathrm{IrH}_{3}\right]_{\mathrm{av}}{ }^{6-} \cdot 2 \mathrm{H}^{-}$.

Distances $(\AA)$ : $\operatorname{Ir}-\mathrm{D}=1.70(4 \times, \quad$ dis $) ; \quad \operatorname{Ir}-\mathrm{Mg}=2.75$; $\mathrm{Ir}-\mathrm{Ir}=4.62 ; \quad \mathrm{Mg}-\mathrm{Mg}=2.76 ; \quad \mathrm{Mg}-\mathrm{D}=2.15 ; \quad \mathrm{Mg}-\mathrm{D}^{-}=$ $1.88 ; \mathrm{D}-\mathrm{D}=2.34$.

\section{$3.25 \mathrm{Mg}_{3} \mathrm{RuH}_{6}$}

From elements by sintering powder mixtures at $1053 \mathrm{~K}$ under 90 bar hydrogen pressure, ${ }^{22}$ powder of rusty-brown color, sensitive to moisture and air; partially disordered structure (Figure 25) from npd on deuteride at room temperature and $15 \mathrm{~K}$ : Cmcm, $Z=4$; Ru surrounded by five $\mathrm{D}$, on average, in disordered octahedral configuration (average $\mathrm{T}$ site symmetry $2 / \mathrm{m}$ ); equatorial sites have $75 \%$ and axial sites full occupancy; surrounded by two $\mathrm{Mg}$ sites in eightfold cubic configuration similar to $\mathrm{Mg}_{3} \mathrm{ReH}_{7}$ (XXIII); three D sites, of which the fourfold is coordinated by two $\mathrm{Mg}$ in linear configuration; no indication for $\mathrm{D}$ ordering down to $15 \mathrm{~K}$; lif: $3 \mathrm{Mg}^{2+} \cdot\left[\mathrm{RuH}_{5}\right]_{\mathrm{av}}{ }^{5-} \cdot \mathrm{H}^{-} ; \mathrm{Ru}^{0}$, $\mathrm{d}^{8}$ (average).

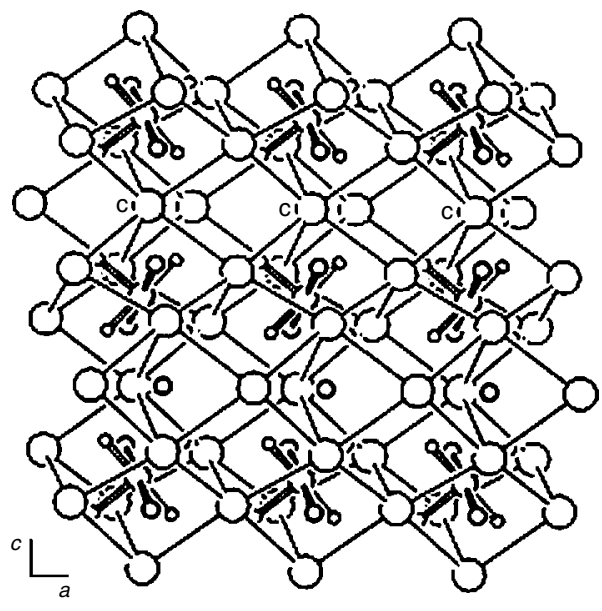

Figure 25 Structure of $\mathrm{Mg}_{3} \mathrm{RuD}_{6}$

Distances $(\AA): \mathrm{Ru}-\mathrm{D}=1.66(4 \times$, dis $), 1.73(2 \times)$; $\mathrm{Ru}-\mathrm{Mg}=2.67 ; \mathrm{Ru}-\mathrm{Ru}=4.79 ; \mathrm{Mg}-\mathrm{Mg}=3.04 ; \mathrm{Mg}-\mathrm{D}=$ $1.91 ; \mathrm{Mg}-\mathrm{D}^{-}=1.87 ; \mathrm{D}-\mathrm{D}=2.29$.

\section{$3.26 \mathrm{Sr}_{8} \mathrm{Rh}_{5} \mathrm{H}_{23}\left(\mathrm{Ca}_{8} \mathrm{Rh}_{5} \mathrm{H}_{23}\right)$}

From alkaline earth hydrides and rhodium metal by sintering powder mixtures at $1073 \mathrm{~K}$ under 5 bar hydrogen pressure; ${ }^{46,47,93}$ powder of black-red $(\mathrm{Sr})$ and black (Ca) color, sensitive to moisture and air; partially disordered, structure intermediate to anion deficient $\mathrm{K}_{2} \mathrm{PtCl}_{6}$ type (II) and perovskite type (Figure 26); from npd on deuterides $(\mathrm{Sr}, \mathrm{Ca})^{46,93}$ at room temperature and $10 \mathrm{~K}: \operatorname{Pm} \overline{3} m, Z=1$; three $\mathrm{D}$ sites of which two are terminal to $\mathrm{Rh}$ with full occupancies and one sixfold is bridging two $\mathrm{Rh}$ with partial occupancy (5/6); $3 \mathrm{Rh}$ sites surrounded in octahedral configurations (site symmetries $m \overline{3} m$ and $4 / m m . m$ ) by six ordered terminal (Rh1), six disordered (5/6 occupancy) bridging (Rh3), and four ordered terminal plus two disordered bridging (Rh2) deuterium sites, the two latter forming a quasiinfinite polynuclear $\left[\mathrm{Rh}_{4} \mathrm{H}_{17}\right]_{n}{ }^{13 n-}$ complex; one $\mathrm{Sr}(\mathrm{Ca})$ site surrounds $\mathrm{Rh}$ in eightfold cubic configuration; no indication for $\mathrm{D}$ ordering down to $10 \mathrm{~K}$; lif: $8 n \mathrm{M}^{2+} \cdot n\left[\mathrm{RhH}_{6}\right]^{3-}$. $\left[\mathrm{Rh}_{4} \mathrm{H}_{17}\right]_{n}{ }^{13 n-}$ mixed valence $\mathrm{Rh}^{\mathrm{I}}\left(\mathrm{d}^{8}\right)$ and $\mathrm{Rh}^{\mathrm{III}}\left(\mathrm{d}^{6}\right)$. Interatomic distances are given in Table 14.

\subsection{7 $\operatorname{LiMg}_{2} \mathrm{RuH}_{7}\left(\mathrm{LiMg}_{2} \mathrm{OsH}_{7}\right)$}

From $\mathrm{LiH}, \mathrm{Mg}$, and $\mathrm{Ru}(\mathrm{Os})$ powders at up to $823 \mathrm{~K}$ under hydrogen pressure of up to 155 bar; ${ }^{94,95}$ light yellow powder; sensitive to air; ordered derivative of $\mathrm{Mg}_{3} \mathrm{ReH}_{7}$ type (XXIII) structure (Figure 27) by npd on deuteride of Ru compound: $P 6_{3} / m m c, Z=2$; octahedral $\left[\mathrm{RuH}_{6}\right]^{4-} 18-$ electron units having T site symmetry $\overline{3} m$, surrounded by six $\mathrm{Mg}^{2+}$ and two $\mathrm{Li}^{+}$in nearly cubic configuration; one $\mathrm{Mg}$ and one Li site; two D sites, of which the twofold is trigonal 


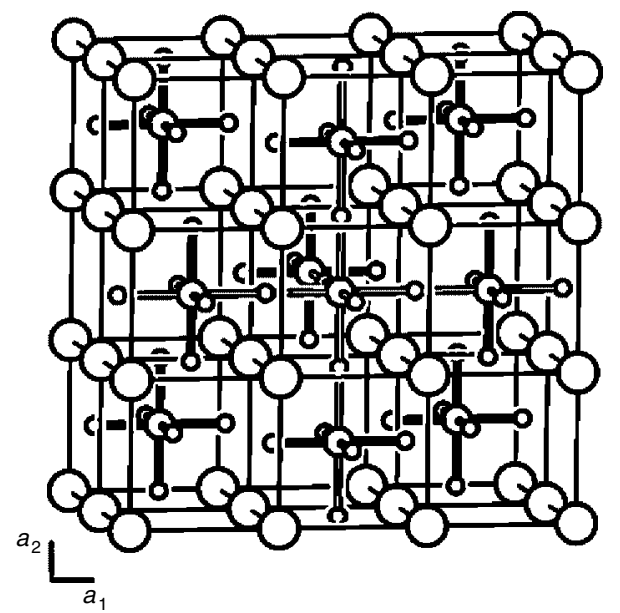

Figure 26 Structure of $\mathrm{Sr}_{8} \mathrm{Rh}_{5} \mathrm{D}_{23}$

Table 14 Interatomic distances $(\AA)$ in $\mathrm{Sr}_{8} \mathrm{Rh}_{5} \mathrm{D}_{23}$ and calcium analog

\begin{tabular}{lcc}
\hline & $\mathrm{M}=\mathrm{Ca}$ & $\mathrm{M}=\mathrm{Sr}$ \\
\hline Rh1-D & $1.69(6 \times)$ & $1.69(6 \times)$ \\
Rh2-D & $1.70(4 \times)$ & $1.70(4 \times)$ \\
& $1.77(2 \times$, dis $)$ & $1.89(2 \times$, dis $)$ \\
Rh3-D & $1.87(6 \times$, dis $)$ & $1.92(6 \times$, dis $)$ \\
Rh-M & 3.14 & 3.29 \\
Rh-Rh & 3.64 & 3.81 \\
M-D & 2.56 & 2.69 \\
M-M & 3.62 & 3.80 \\
D-D & 2.39 & 2.38 \\
\hline
\end{tabular}

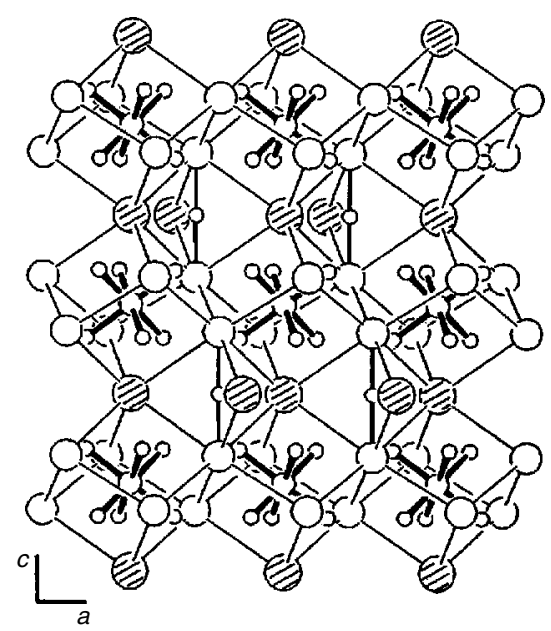

Figure 27 Structure of $\mathrm{LiMg}_{2} \mathrm{RuD}_{7}$

bipyramidal coordinated with two short linear $\mathrm{Mg}-\mathrm{D}$ and three long triangular $\mathrm{Li}-\mathrm{D}$ bonds; lif: $\mathrm{Li}^{+} \cdot 2 \mathrm{Mg}^{2+} \cdot\left[\mathrm{RuH}_{6}\right]^{4-} \cdot \mathrm{H}^{-}$; $\mathrm{Ru}^{\mathrm{II}}, \mathrm{d}^{6}$.

Distances $(\AA): \quad \mathrm{Ru}-\mathrm{D}=1.69(6 \times) ; \quad \mathrm{Ru}-\mathrm{Mg}=2.83$; $\mathrm{Ru}-\mathrm{Li}=2.67 ; \mathrm{Ru}-\mathrm{Ru}=4.70 ; \mathrm{Mg}-\mathrm{Li}=3.29 ; \mathrm{Mg}-\mathrm{Mg}=$

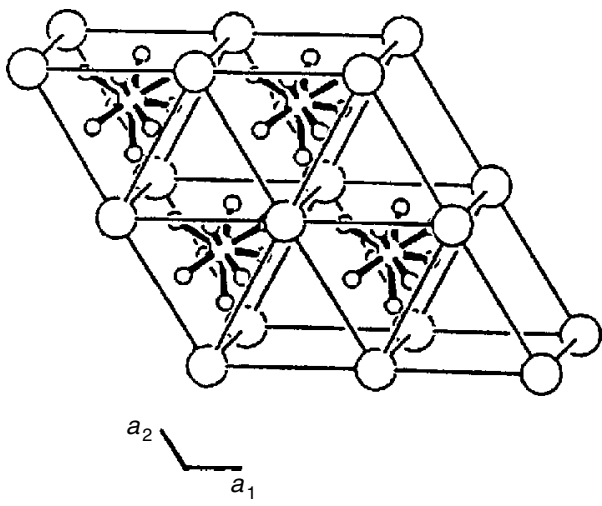

Figure 28 Structure of $\mathrm{BaReH}_{9}$

$3.17 ; \mathrm{Li}-\mathrm{D}=2.14 ; \mathrm{Li}-\mathrm{D}^{-}=2.71 ; \mathrm{Mg}-\mathrm{D}=2.27 ; \mathrm{Mg}-\mathrm{D}^{-}=$ $1.85 ; \mathrm{D}-\mathrm{D}=2.34$.

\subsection{BaReH9}

From di-sodium salt $\mathrm{Na}_{2} \mathrm{ReH}_{9}$ (obtained from reaction of $\mathrm{KReO}_{4}$ with $\mathrm{K}$ metal in ethylene diamine-water solution) converted to the di-potassium salt by way of the $\mathrm{Ba}$ salt; ${ }^{96}$ white powder, transparent crystals, unstable in moist air; ordered structure (Figure 28) from single-crystal X-ray diffraction, and IR and NMR data on hydride: ${ }^{97} \mathrm{~Pb}_{3} / \mathrm{mmc}$, $Z=2$; Re site forming ttp-[ $\left[\mathrm{ReH}_{9}\right]^{2-}$ 18-electron complexes with site symmetry $\overline{6} \mathrm{~m} 2$; surrounded by Ba cations in NiAs type configuration; two $\mathrm{H}$ sites (not refined); lif: $\mathrm{Ba}^{2+} \cdot\left[\mathrm{ReH}_{9}\right]^{2-} ; \mathrm{Re}^{\mathrm{VII}}, \mathrm{d}^{0}$; electronic band structure by APW calculations. $^{98}$

Interatomic distances $(\AA): \mathrm{Re}-\mathrm{H} \sim 1.69^{\mathrm{a}} \quad(3 \times \quad$ and $6 \times)$; $\mathrm{Re}-\mathrm{Ba}=3.84 ; \mathrm{Re}-\mathrm{Re}=5.29 ; \mathrm{Ba}-\mathrm{Ba}=4.66 ; \mathrm{Ba}-\mathrm{H}=$ $2.71^{\mathrm{a}}, 2.86 ; \mathrm{H}-\mathrm{H} \sim 1.97^{\mathrm{a}}$ (distances not refined).

\subsection{9 $\mathrm{K}_{2} \mathrm{ZnH}_{4}\left(\mathrm{Rb}_{2} \mathrm{ZnH}_{4}, \mathrm{Cs}_{2} \mathrm{ZnH}_{4}, \mathrm{Sr}_{2} \mathrm{PdH}_{4}, \mathrm{Ba}_{2} \mathrm{PdH}_{4}\right.$, $\left.\mathrm{Eu}_{2} \mathrm{PdH}_{4}\right)$}

$\mathrm{M}_{2} \mathrm{ZnH}_{4}(\mathrm{M}=\mathrm{K}, \mathrm{Rb}, \mathrm{Cs})$ from elements by sintering powder mixtures at $783-793 \mathrm{~K}$ under 120 bar hydrogen pressure, ${ }^{99,100} \mathrm{M}_{2} \mathrm{PdH}_{4}\left(\mathrm{M}=\mathrm{Sr}, \mathrm{Ba},{ }^{101} \mathrm{Eu}^{102}\right)$, from elements gray powder; sensitive to air; ordered structure (Figure 29) by npd on deuteride: Pnma, $Z=2 ; \beta-\mathrm{K}_{2} \mathrm{SO}_{4}$ type structure, tetrahedral $\left[\mathrm{ZnH}_{4}\right]^{2-}$ 18-electron complexes with $\mathrm{T}$ site symmetry $m$ surrounded by two M sites in eightfold distorted square antiprismatic configuration; three $\mathrm{D}$ sites; evidence for nonconducting behavior of $\mathrm{Sr}_{2} \mathrm{PdH}_{4}$ and $\mathrm{Ba}_{2} \mathrm{PdH}_{4}$ (measurement on powdered hydrides); ${ }^{101}$ electronic band structure on $\mathrm{Eu}_{2} \mathrm{PdH}_{4} ;{ }^{103}$ lif: $2 \mathrm{~K}^{+} \cdot\left[\mathrm{ZnH}_{4}\right]^{2-} ; \mathrm{Zn}^{\mathrm{II}}, \mathrm{d}^{10}$; $2 \mathrm{M}^{2+} \cdot\left[\mathrm{TH}_{4}\right]^{4-}, \mathrm{Pd}^{0}, \mathrm{~d}^{10}$. Interatomic distances are given in Table 15. 


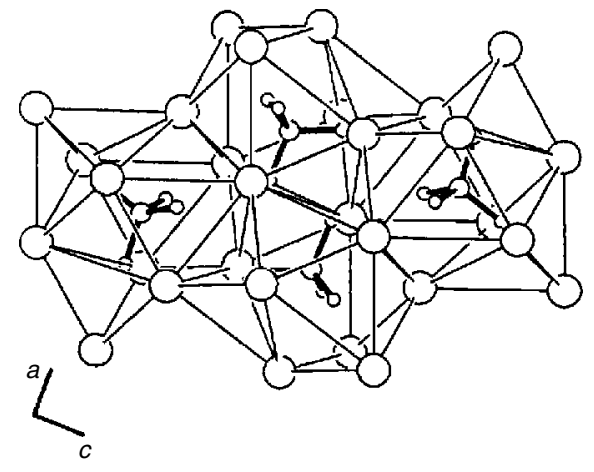

Figure 29 Structure of $\mathrm{K}_{2} \mathrm{ZnD}_{4}$

Table 15 Interatomic distances $(\AA)$ in $\mathrm{K}_{2} \mathrm{ZnD}_{4}$ and analogs

\begin{tabular}{ccccc}
\hline & & $\mathrm{M}=\mathrm{K}$ & $\mathrm{M}=\mathrm{Rb}$ & $\mathrm{M}=\mathrm{Cs}$ \\
\hline $\mathrm{T}=\mathrm{Zn}$ & $\mathrm{Zn}-\mathrm{D}$ & $1.63-1.66$ & $1.66-1.68$ & $1.67-1.69$ \\
& $\mathrm{Zn}-\mathrm{M}$ & 3.31 & 3.49 & 3.59 \\
& $\mathrm{M}-\mathrm{D}$ & 2.72 & 2.89 & 2.97 \\
& $\mathrm{Zn}-\mathrm{Zn}$ & 4.97 & 5.24 & 5.56 \\
& $\mathrm{M}-\mathrm{M}$ & 3.86 & 3.94 & 4.12 \\
& $\mathrm{D}-\mathrm{D}$ & 2.69 & 2.72 & 2.73 \\
& & & & \\
$\mathrm{~T}=\mathrm{Pd}$ & $\mathrm{M}=\mathrm{Sr}$ & $\mathrm{M}=\mathrm{Ba}$ & $\mathrm{M}=\mathrm{Eu}$ \\
& $\mathrm{Pd}-\mathrm{D}$ & $1.76-1.81$ & $1.78-1.81$ & $1.67-1.83$ \\
& $\mathrm{Pd}-\mathrm{M}$ & 3.09 & 3.23 & 3.07 \\
& $\mathrm{M}-\mathrm{D}$ & 2.49 & 2.72 & 2.54 \\
& $\mathrm{Pd}-\mathrm{Pd}$ & 4.83 & 5.07 & 4.78 \\
& $\mathrm{M}-\mathrm{M}$ & 3.65 & 3.78 & 3.57 \\
& $\mathrm{D}-\mathrm{D}$ & 2.58 & 2.80 & 2.69 \\
\hline
\end{tabular}

\section{$3.30 \mathrm{~K}_{3} \mathrm{ZnH}_{5}\left(\mathrm{Rb}_{3} \mathrm{ZnH}_{5}, \mathrm{Cs}_{3} \mathrm{ZnH}_{5}, \mathrm{Cs}_{3} \mathrm{CdH}_{5}, \mathrm{~K}_{3} \mathrm{MnH}_{5}\right.$, $\left.\mathrm{Rb}_{3} \mathrm{MnH}_{5}, \mathrm{Cs}_{3} \mathrm{MnH}_{5}\right)$}

$\mathrm{M}_{3} \mathrm{ZnH}_{5}(\mathrm{M}=\mathrm{K}, \mathrm{Rb}, \mathrm{Cs})$ from elements by sintering powder mixtures at $783-793 \mathrm{~K}$ under 120 bar hydrogen pressure; 104,105 $\mathrm{M}_{3} \mathrm{MnH}_{5}(\mathrm{M}=\mathrm{Sr}, \mathrm{Ba})$ from alkali metal hydrides and $\mathrm{Mn}$ powder at $875 \mathrm{~K}$ and $3 \mathrm{kbar} \mathrm{H}_{2}$ gas pressure, ${ }^{106,107} \mathrm{Cs}_{3} \mathrm{CdH}_{5}{ }^{108}$ from elements, gray ( $\left.\mathrm{Zn}, \mathrm{Cd}\right)$ and rose colored $(\mathrm{Mn})$ powders; sensitive to air; ordered structure (Figure 30) by npd on deuteride: $I 4 / \mathrm{mcm}, Z=4$; $\mathrm{Cs}_{3} \mathrm{CoCl}_{5}$ type structure, tetrahedral $\left[\mathrm{TH}_{4}\right]^{2-}$ 18-electron complexes with $\mathrm{T}$ site symmetry $\overline{4} 2 \mathrm{~m}$ surrounded by two $M$ sites in an eightfold configuration; two D sites, of which the fourfold is surrounded by $\mathrm{M}$ in octahedral configuration; arrangement of $\mathrm{H}^{-}$and $\left[\mathrm{TH}_{4}\right]^{2-}$ complexes similar to that in $\mathrm{K}_{3} \mathrm{PtH}_{5}$ (VIII); vibrational spectroscopy of $\mathrm{Rb}_{3} \mathrm{ZnH}_{5} ;{ }^{\mathbf{5 4}}$ lif: $3 \mathrm{M}^{+} \cdot\left[\mathrm{TH}_{4}\right]^{2-} \cdot \mathrm{H}^{-} ; \mathrm{Zn}^{\mathrm{II}}, \mathrm{d}^{10} ; \mathrm{Cd}^{\mathrm{II}}, \mathrm{d}^{10} ; \mathrm{Mn}^{\mathrm{II}}, \mathrm{d}^{5} ; \mathrm{Mn}$ compounds order magnetically below $50 \mathrm{~K} .{ }^{9}$ Interatomic distances are given in Table 16.

\subsection{1 $\mathrm{Ba}_{3} \mathrm{Ir}_{2} \mathrm{H}_{12}$}

From powder mixtures of $\mathrm{BaH}_{2}$ and $\mathrm{Ir}$ at $837 \mathrm{~K}$ and 80 bar hydrogen pressure; ${ }^{\mathbf{1 0 9}}$ white powder, sensitive to air;

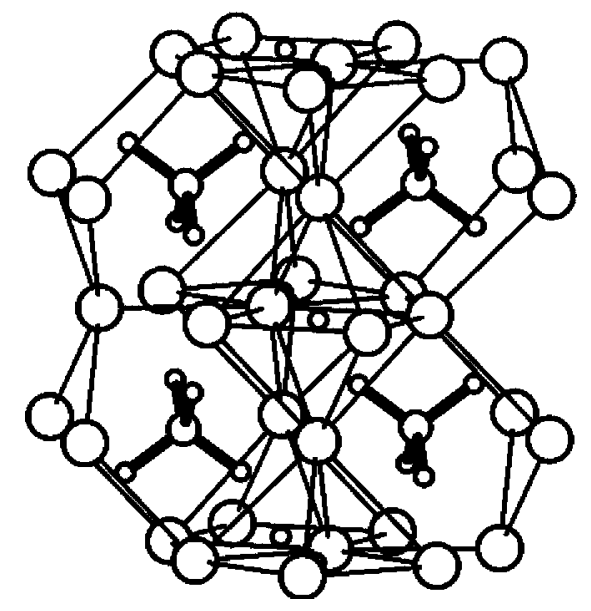

Figure 30 Structure of $\mathrm{K}_{3} \mathrm{ZnD}_{5}$

Table 16 Interatomic distances $(\AA)$ in $\mathrm{K}_{3} \mathrm{ZnD}_{5}$ and analogs

\begin{tabular}{clccc}
\hline & & $\mathrm{M}=\mathrm{K}$ & $\mathrm{M}=\mathrm{Rb}$ & $\mathrm{M}=\mathrm{Cs}$ \\
\hline $\mathrm{T}=\mathrm{Zn}$ & Zn-D & 1.66 & 1.66 & 1.68 \\
& Zn-M & 3.41 & 3.56 & 3.72 \\
& $\mathrm{M}-\mathrm{D}$ & 2.75 & 2.94 & 3.15 \\
& $\mathrm{M}-\mathrm{D}^{-}$ & 2.78 & 2.91 & 3.04 \\
& Zn-Zn & 5.36 & 5.61 & 5.87 \\
& $\mathrm{M}-\mathrm{M}$ & 3.92 & 4.10 & 4.26 \\
$\mathrm{~T}=\mathrm{Cd}$ & $\mathrm{D}-\mathrm{D}$ & 2.66 & 2.69 & 2.72 \\
& $\mathrm{Cd}-\mathrm{D}$ & & & 1.82 \\
& $\mathrm{Cd}-\mathrm{M}$ & & & 3.83 \\
& $\mathrm{M}-\mathrm{D}$ & & & 3.11 \\
& $\mathrm{M}-\mathrm{D}-$ & & & 3.08 \\
& $\mathrm{Cd}-\mathrm{Cd}$ & & & 5.96 \\
$\mathrm{~T}=\mathrm{Mn}$ & & & 4.35 \\
& $\mathrm{M}-\mathrm{M}$ & & & 2.93 \\
& $\mathrm{D}-\mathrm{D}$ & & 1.76 & 1.79 \\
& $\mathrm{Mn}-\mathrm{D}$ & 1.75 & 3.69 & 3.82 \\
& $\mathrm{Mn}-\mathrm{M}$ & 3.55 & 2.88 & 3.11 \\
& $\mathrm{M}-\mathrm{D}$ & 2.73 & 2.92 & 3.07 \\
& $\mathrm{M}-\mathrm{D}^{-}$ & 2.80 & 5.66 & 5.94 \\
& $\mathrm{Mn}-\mathrm{Mn}$ & 5.43 & & 4.34 \\
& $\mathrm{M}-\mathrm{M}$ & 3.96 & 4.12 & 2.87 \\
\hline & $\mathrm{D}-\mathrm{D}$ & 2.78 & 2.85 &
\end{tabular}

ordered structure (Figure 31) from npd on deuteride at room temperature; $P \overline{3} m 1, Z=1$; octahedral $\left[\mathrm{IrH}_{6}\right]^{3-}$ 18-electron complex with Ir site symmetry $3 m$; surrounded by two Ba sites in irregular sevenfold coordination; two D sites; lif: $3 \mathrm{Ba}^{2+} \cdot 2\left[\mathrm{IrH}_{6}\right]^{3-}, \mathrm{Ir}^{\mathrm{III}}, \mathrm{d}^{6}$.

Distances $(\AA): \operatorname{Ir}-\mathrm{D}=1.60(3 \times)$ and $1.76(3 \times) ; \mathrm{Ir}-\mathrm{Ba}=$ $3.30 ; \mathrm{Ir}-\mathrm{Ir}=5.43 ; \mathrm{Ba}-\mathrm{D}=2.72 ; \mathrm{D}-\mathrm{D}=2.25$.

\subsection{2 $\mathrm{Ca}_{4} \mathrm{Mg}_{4} \mathrm{Co}_{3} \mathrm{H}_{19}\left(\mathrm{Yb}_{4} \mathrm{Mg}_{4} \mathrm{Co}_{3} \mathrm{H}_{19}\right)$}

From mixtures of arc-melted binary alloys (CaMg, YbMg) and Co rods, by adding $\mathrm{LiH}$ as a flux, at up to $800 \mathrm{~K}$ and 155 bar hydrogen pressure; ${ }^{110}$ black powders, Ca compound containing brownish translucent single crystals; partially 


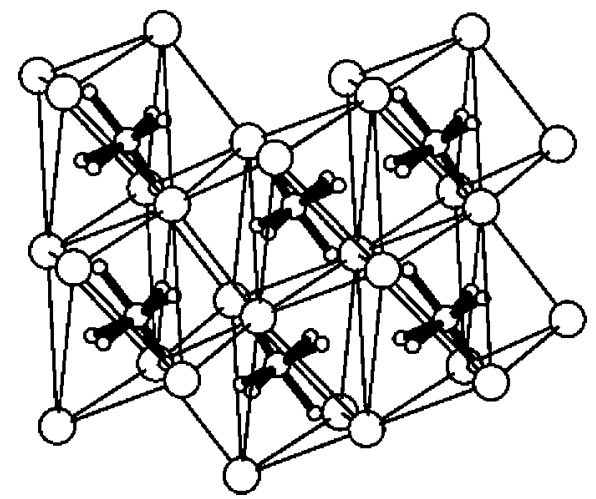

Figure 31 Structure of $\mathrm{Ba}_{3} \mathrm{Ir}_{2} \mathrm{D}_{12}$

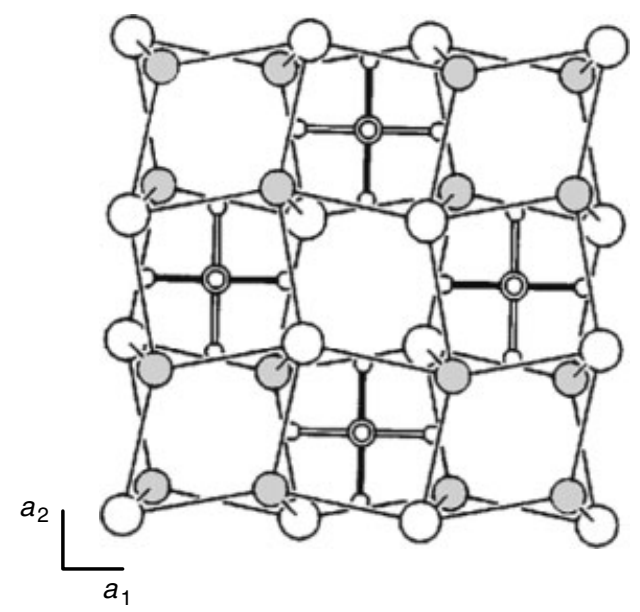

Figure 32 Structure of $\mathrm{Ca}_{4} \mathrm{Mg}_{4} \mathrm{Co}_{3} \mathrm{D}_{19}$

disordered structures (Figure 32) from npd on deuterides and single-crystal $\mathrm{X}$ ray on $\mathrm{Ca}_{4} \mathrm{Mg}_{4} \mathrm{Co}_{3} \mathrm{H}_{19} ; P \overline{4} 3 m, Z=1$; derives from $\mathrm{Fe}$ analog $\mathrm{Ca}_{4} \mathrm{Mg}_{4} \mathrm{Fe}_{3} \mathrm{H}_{22}$ (XX) by substitution of oct $\left[\mathrm{FeH}_{6}\right]^{4-}$ by spy $\left[\mathrm{CoH}_{5}\right]^{4-} 18$-electron complexes; apical Co ligands disordered (half occupancy), Co site symmetry $\overline{4} 2 . m$, surrounded by $\mathrm{Mg}$ and $\mathrm{Ca}(\mathrm{Yb})$ sites in deformed cubic configuration; three $\mathrm{D}$ sites, of which one anionic $\mathrm{D}^{-}$ tetrahedral surrounded by one $\mathrm{Mg}^{2+}$ and three $\mathrm{M}^{2+}$; derives from $\mathrm{Mg}_{2} \mathrm{CoH}_{5}$ (III) by partial substitution of $\mathrm{Mg}$ by $\mathrm{Ca}$, and of every fourth $\left[\mathrm{CoH}_{5}\right]^{4-}$ by $4 \mathrm{H}^{-}$; lif: $4 \mathrm{M}^{2+} .4 \mathrm{Mg}^{2+}$. $3\left[\mathrm{CoH}_{5}\right]_{\mathrm{av}}{ }^{4-} \cdot 4 \mathrm{H}^{-} ; \mathrm{Co}^{\mathrm{I}}, \mathrm{d}^{8}$. Interatomic distances are given in Table 17.

\subsection{KNaReH9}

Translucent crystals obtained from di-sodium salt $\mathrm{Na}_{2} \mathrm{ReH}_{9}{ }^{96}$ by slow evaporation of a solution in $\mathrm{KOH}$; unstable in moist air; ordered structure (Figure 33) from single-crystal X-ray diffraction at $193 \mathrm{~K}$, and IR data: ${ }^{111}$ Pnma, $Z=4$; Re site forming ttp- $\left[\mathrm{ReH}_{9}\right]^{2-} 18$-electron complexes with site symmetry $m$; surrounded by $\mathrm{K}$ and $\mathrm{Na}$ cations in
Table 17 Interatomic distances $(\AA)$ in $\mathrm{Ca}_{4} \mathrm{Mg}_{4} \mathrm{Co}_{3} \mathrm{D}_{19}$ and ytterbium analog

\begin{tabular}{lcc}
\hline & $\mathrm{M}=\mathrm{Ca}$ & $\mathrm{M}=\mathrm{Yb}$ \\
\hline Co-D & $1.55-1.59$ & $1.55-1.58$ \\
Co-Mg & 2.75 & 2.74 \\
Co-M & 3.11 & 3.10 \\
Mg-D & 1.91 & 1.89 \\
Mg-D & 1.96 & 1.94 \\
M-D & 2.44 & 2.43 \\
M-D & 2.35 & 2.36 \\
Co-Co & 4.72 & 4.71 \\
Mg-Mg & 3.72 & 3.67 \\
Mg-M & 3.42 & 3.40 \\
D-D & 2.19 & 2.19 \\
\hline
\end{tabular}

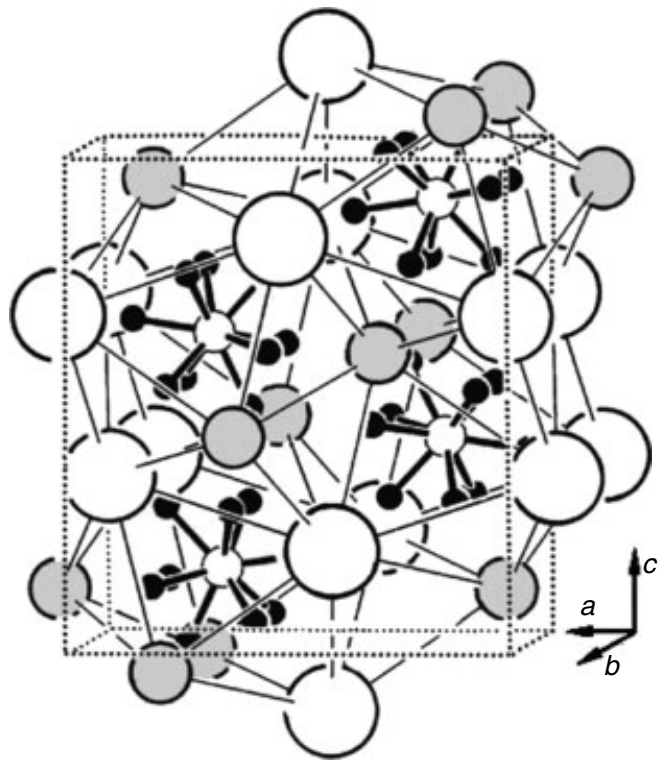

Figure 33 Structure of $\mathrm{KNaReH}_{9}$

TiNiSi type metal configuration; six $\mathrm{H}$ sites (not refined); lif: $\mathrm{K}^{+} \cdot \mathrm{Na}^{+} \cdot\left[\mathrm{ReH}_{9}\right]^{2-} ; \mathrm{Re}^{\mathrm{VII}}, \mathrm{d}^{0}$.

Interatomic distances $(\AA): \mathrm{Re}-\mathrm{H}=1.69-1.71^{\mathrm{a}} ; \mathrm{Re}-\mathrm{Na}=$ 3.35; $\mathrm{Re}-\mathrm{K}=3.69 ; \mathrm{Re}-\mathrm{Re}=5.33 ; \mathrm{Na}-\mathrm{Na}=4.00 ; \mathrm{Na}-\mathrm{K}=$ $4.01 ; \mathrm{K}-\mathrm{K}=4.49 ; \mathrm{Na}-\mathrm{H}=2.33^{\mathrm{a}} ; \mathrm{K}-\mathrm{H}=2.61^{\mathrm{a}} ; \mathrm{H}-\mathrm{H}=$ $1.73^{\mathrm{a}}$ (not refined).

\section{$3.34 \quad \mathrm{Li}_{5} \mathrm{Pt}_{2} \mathrm{H}_{9}$}

From reaction of $\mathrm{LiH}$ and $\mathrm{Pt}$ at 1250 bar $\mathrm{H}_{2}$ pressure and $820 \mathrm{~K} ;{ }^{112}$ anthracite colored caked solid, sensitive to air and moisture; ordered structure (Figure 34) from npd on deuteride: $I 4 / \mathrm{mcm}, Z=4$; contains $\left[\mathrm{Pt}_{2} \mathrm{H}_{9}\right]^{5-}$ dimer with $m . m m$ symmetry in which four $\mathrm{D}$ surround each $\mathrm{Pt}$ in spl configuration and one $\mathrm{D}$ bridges two $\mathrm{Pt} ; 2 \mathrm{Li}$ sites; diamagnetic; lif: $5 \mathrm{M}^{+} \cdot\left[\mathrm{T}_{2} \mathrm{H}_{9}\right]^{5-} ; \mathrm{Pt}^{\mathrm{II}}, \mathrm{d}^{8}$.

Distances $(\AA): \mathrm{Pt}-\mathrm{D}=1.64(4 \times), 1.85 ; \mathrm{Pt}-\mathrm{Li}=2.64$; $\mathrm{Pt}-\mathrm{Pt}=3.71 ; \mathrm{Li}-\mathrm{D}=2.13 ; \mathrm{D}-\mathrm{D}=2.28$. 


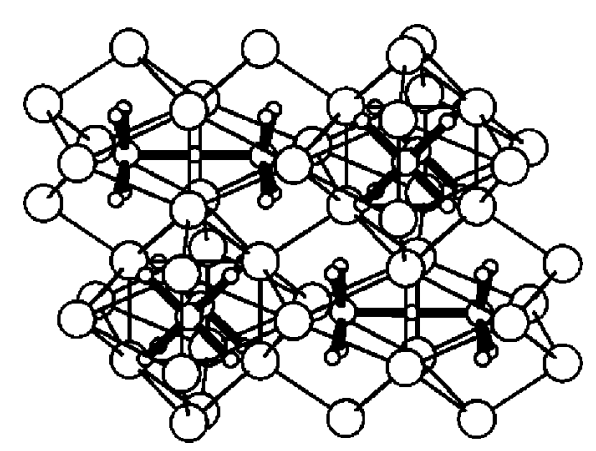

Figure 34 Structure of $\mathrm{Li}_{5} \mathrm{Pt}_{2} \mathrm{D}_{9}$

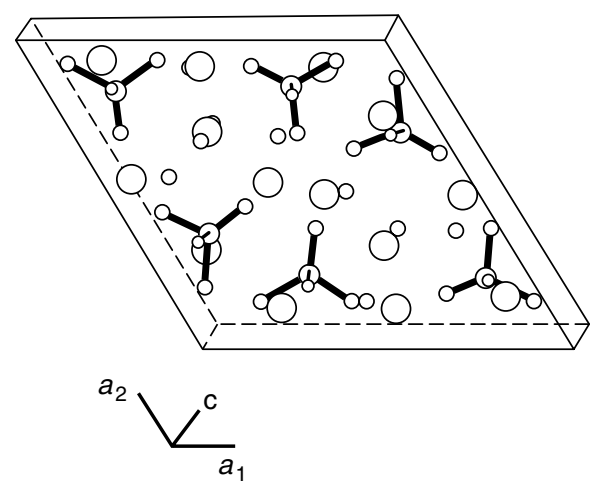

Figure 35 Structure of $\mathrm{Ba}_{7} \mathrm{Cu}_{3} \mathrm{D}_{17}$

\subsection{5 $\quad \mathrm{Ba}_{7} \mathrm{Cu}_{3} \mathrm{H}_{17}$}

By hydrogenation of $\mathrm{Ba}-\mathrm{Cu}$ alloys at 10 bar $\mathrm{H}_{2}$ pressure and room temperature, followed by annealing at $643 \mathrm{~K}$ under 160 bar $\mathrm{H}_{2}$ pressure; ${ }^{113}$ dark brownish powder sensitive to air and moisture; ordered structure (Figure 35) from npd on deuteride: $P 31 c, Z=2$; contains distorted 18-electron tet$\left[\mathrm{CuD}_{4}\right]^{3-}$ complexes (symmetry 1 ) surrounded by nine $\mathrm{Ba}$ (three sites) in ttp configuration; seven D sites, of which three anionic $\mathrm{D}^{-}$tetrahedral surrounded by four $\mathrm{M}^{2+}$; lif: $7 \mathrm{Ba}^{2+} .3\left[\mathrm{CuH}_{4}\right]^{3-} .5 \mathrm{H}^{-} ; \mathrm{Cu}^{\mathrm{I}}, \mathrm{d}^{10}$.

Distances $(\AA): \quad \mathrm{Cu}-\mathrm{D}=1.64-1.75 ; \quad \mathrm{Cu}-\mathrm{Ba}=3.19$; $\mathrm{Cu}-\mathrm{Cu}=5.07 ; \mathrm{Ba}-\mathrm{D}=2.36 ; \mathrm{Ba}-\mathrm{D}^{-}=2.59 ; \mathrm{D}-\mathrm{D}=1.94$.

\subsection{6 $\mathrm{LiMg}_{4} \mathrm{Os}_{2} \mathrm{H}_{13}\left(\mathrm{LiMg}_{4} \mathrm{Ru}_{2} \mathrm{H}_{13}\right)$}

By sintering of mixtures of $\mathrm{LiH}, \mathrm{Mg}$, and $\mathrm{Os}(\mathrm{Ru})$ powders at up to $833 \mathrm{~K}$ under hydrogen pressure between 70 and 155 bar; ${ }^{95}$ powders having light green (low pressure) and grey (high pressure) color; sensitive to air; Os sample pyrophoric; ordered structure (Figure 36 ) by npd on deuteride of Os compound: $P 6_{3} / m m c, Z=2$; octahedral $\left[\mathrm{OsH}_{6}\right]^{4-}$ 18-electron units having $\mathrm{T}$ site symmetry $3 m$ surrounded by six $\mathrm{Mg}^{2+}$ and two $\mathrm{Li}^{+}$in nearly cubic configuration; one $\mathrm{Mg}$ and one $\mathrm{Li}$ site; three $\mathrm{D}$ sites, of which an anionic $\mathrm{D}^{-}$in trigonal bipyramidal coordination with two

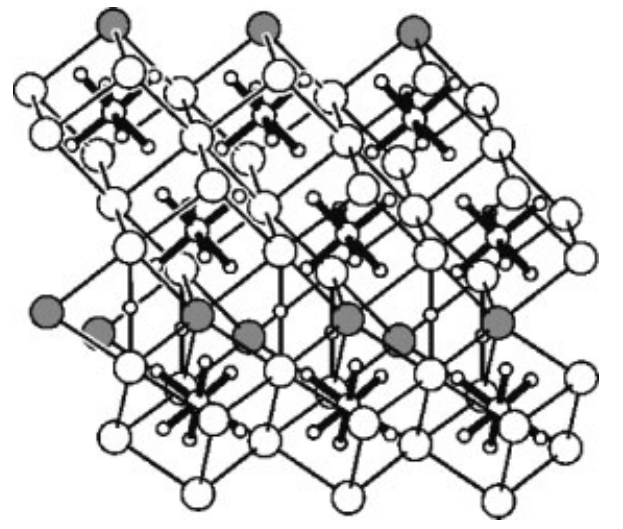

Figure 36 Structure of $\mathrm{LiMg}_{4} \mathrm{Os}_{2} \mathrm{D}_{13}$

short linear $\mathrm{Mg}-\mathrm{D}$ and three long triangular $\mathrm{Li}-\mathrm{D}$ bonds; structure derives from $\mathrm{Mg}_{2} \mathrm{OsH}_{6}$ by intercalation of $\mathrm{LiH}$ sheets; intermediate between $\mathrm{Mg}_{2} \mathrm{OsH}_{6}$ (II) and $\mathrm{LiMg}_{2} \mathrm{OsH}_{7}$ (XXVII); lif: $\mathrm{Li}^{+} \cdot 4 \mathrm{Mg}^{2+} \cdot 2\left[\mathrm{TH}_{6}\right]^{4-} \cdot \mathrm{H}^{-} ; \mathrm{Os}^{\mathrm{II}}, \mathrm{d}^{6}$.

Distances $(\AA):$ Os-D $=1.69(3 \times)$ and $1.70(3 \times)$; Os- $-\mathrm{Li}=2.69 ;$ Os- $\mathrm{Mg}=2.85 ; \mathrm{Mg}-\mathrm{Li}=3.31 ; \mathrm{Mg}-\mathrm{Mg}=$ $3.26 ; \mathrm{Li}-\mathrm{D}=2.13 ; \mathrm{Li}-\mathrm{D}^{-}=2.73 ; \mathrm{Mg}-\mathrm{D}=2.24 ; \mathrm{Mg}-\mathrm{D}^{-}=$ $1.87 ; \mathrm{D}-\mathrm{D}=2.31$.

\subsection{7 $\quad \mathrm{Li}_{2} \mathrm{PtH}_{2}$}

By thermal decomposition of $\mathrm{Li}_{5} \mathrm{Pt}_{2} \mathrm{H}_{9}$ (XXXIV) at $493 \mathrm{~K}$ under argon; ${ }^{114}$ ordered structure (Figure 37 ) from npd on deuteride: Immm, $Z=2$; contains linear $\left[\mathrm{PtD}_{2}\right]^{2-}$ 14-electron complexes with mmm symmetry; orthorhombic distortion of tetragonal $\mathrm{Pd}$ analog $\mathrm{Li}_{2} \mathrm{PdH}_{2}$ (VII); lif: $2 \mathrm{Li}^{+} \cdot\left[\mathrm{PtH}_{2}\right]^{2-} ; \mathrm{Pt}^{0}$, $\mathrm{d}^{10}$.

Distances $(\AA): \mathrm{Pt}-\mathrm{D}=1.64(2 \times) ; \mathrm{Pt}-\mathrm{Pt}=2.99 ; \mathrm{Pt}-\mathrm{Li}=$ 2.61; Li-Li $=2.75 ; \mathrm{Li}-\mathrm{D}=2.01 ; \mathrm{D}-\mathrm{D}=2.82$.

\subsection{8 $\mathrm{BaMg}_{2} \mathrm{RuH}_{8}\left(\mathrm{BaMg}_{2} \mathrm{OsH}_{8}\right)$}

By sintering powder mixtures of $\mathrm{BaMg}_{2}$ alloy, $\mathrm{BaH}_{2}$, and $\mathrm{Ru}$ with $\mathrm{LiH}$ flux at temperatures of up to $803 \mathrm{~K}$ and $\mathrm{H}_{2}$ pressures of up to $150 \mathrm{bar}^{115}$ greenish powder;

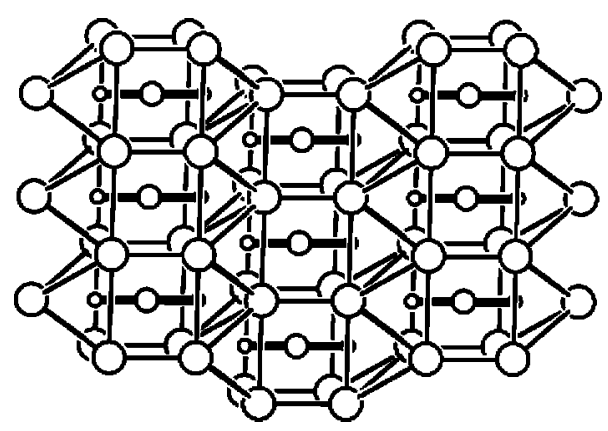

Figure 37 Structure of $\mathrm{Li}_{2} \mathrm{PtD}_{2}$ 


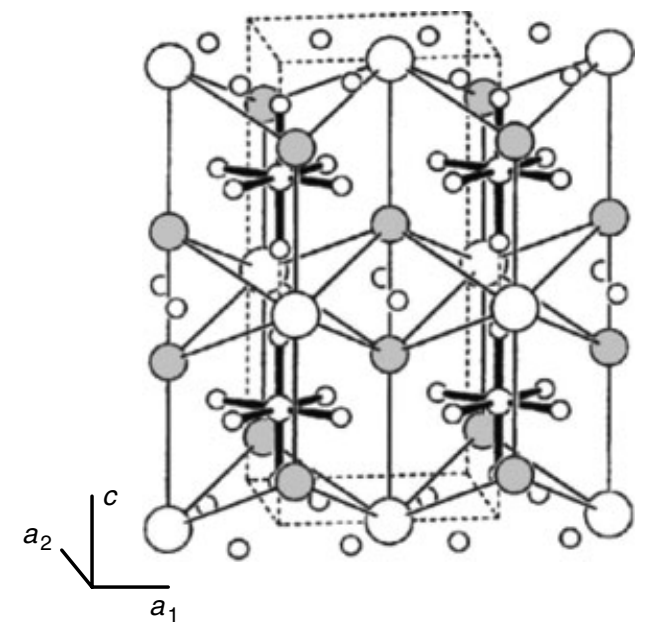

Figure 38 Structure of $\mathrm{BaMg}_{2} \mathrm{RuD}_{8}$

Table 18 Interatomic distances $(\AA)$ in $\mathrm{BaMg}_{2} \mathrm{RuD}_{8}$ and osmium analog

\begin{tabular}{lcc}
\hline & $\mathrm{T}=\mathrm{Ru}$ & $\mathrm{T}=$ Os \\
\hline $\mathrm{T}-\mathrm{D}$ & $1.68-1.72$ & $1.70-1.72$ \\
$\mathrm{~T}-\mathrm{Mg}$ & 2.74 & 2.75 \\
$\mathrm{~T}-\mathrm{Ba}$ & 3.67 & 3.67 \\
$\mathrm{Mg}-\mathrm{D}$ & 2.10 & 2.12 \\
$\mathrm{Mg}-\mathrm{D}^{-}$ & 1.99 & 1.97 \\
$\mathrm{Ba}-\mathrm{D}$ & 2.68 & 2.68 \\
$\mathrm{Ba}-\mathrm{D}^{-}$ & 2.77 & 2.77 \\
$\mathrm{~T}-\mathrm{T}$ & 4.96 & 4.97 \\
$\mathrm{Mg}-\mathrm{Mg}$ & 3.08 & 3.03 \\
$\mathrm{Mg}-\mathrm{Ba}$ & 3.83 & 3.82 \\
$\mathrm{D}-\mathrm{D}$ & 2.04 & 2.02 \\
\hline
\end{tabular}

ordered structure (Figure 38) by npd on deuterides of $\mathrm{Ru}$ and Os compounds at room temperature: $P 4_{2} / m m c, Z=2$; octahedral $\left[\mathrm{TH}_{6}\right]^{4-} 18$-electron units having $\mathrm{T}$ site symmetry $\overline{4} m 2$ surrounded by four $\mathrm{Mg}^{2+}$ and four $\mathrm{Ba}^{2+}$ forming two interpenetrating tetrahedral; similarity to iron analog $\mathrm{BaMg}_{2} \mathrm{FeH}_{8}$ (XVI); three D sites, of which one hydridic $\mathrm{D}^{-}$is tetrahedral coordinated by two $\mathrm{Ba}$ and two $\mathrm{Mg}$; lif: $\mathrm{Ba}^{2+} \cdot 2 \mathrm{Mg}^{2+} \cdot\left[\mathrm{TH}_{6}\right]^{4-} \cdot 2 \mathrm{H}^{-} ; \mathrm{T}^{\mathrm{II}}, \mathrm{d}^{6}$. Interatomic distances are given in Table 18.

\subsection{9 $\mathrm{NaBaPdH}_{3}$}

By sintering of compacted powder mixtures of $\mathrm{NaH}, \mathrm{BaH}_{2}$, and $\mathrm{Pd}$ at temperatures of up to $783 \mathrm{~K}$ under $\mathrm{H}_{2}$ pressures of 90 bar; ${ }^{116}$ solid having metallic luster, sensitive to air and moisture, contains ruby-red single crystals; ordered structure (Figure 39) from single-crystal X-ray diffraction on hydride and npd on deuteride: $P 6_{3} / m m c, Z=2$; contains trigonal planar $\left[\mathrm{PdH}_{3}\right]^{3-}$ 16-electron complexes with $\overline{6} m 2$ symmetry; surrounded by five $\mathrm{Ba}$ in trigonal bipyramidal configuration; one $\mathrm{Pd}$ site, one $\mathrm{Na}$ site, one $\mathrm{Ba}$ site and one $\mathrm{D}$ site;

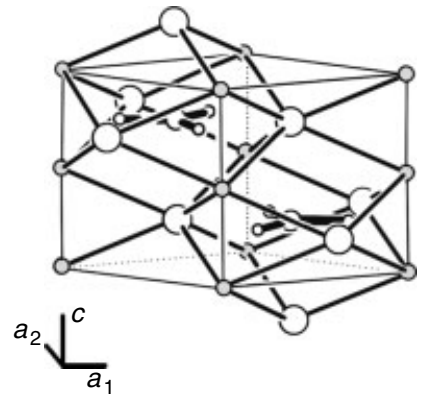

Figure 39 Structure of $\mathrm{NaBaPdD}_{3}$

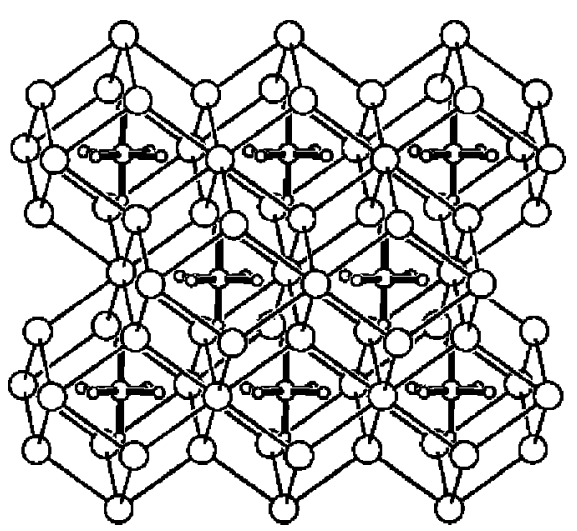

Figure 40 Structure of $\mathrm{K}_{3} \mathrm{ReD}_{6}$

lif: $\mathrm{Na}^{+} \cdot \mathrm{Ba}^{2+} \cdot\left[\mathrm{PdH}_{3}\right]^{3-} ; \mathrm{Pd}^{0}, \mathrm{~d}^{10}$ (supported by ac-magnetic susceptibility measurements).

Distances $\quad(\AA): \quad \mathrm{Pd}-\mathrm{D}=1.72(3 \times) ; \quad \mathrm{Pd}-\mathrm{Ba}=3.04$; $\mathrm{Pd}-\mathrm{Na}=3.81 ; \quad \mathrm{Pd}-\mathrm{Pd}=4.63 ; \quad \mathrm{Ba}-\mathrm{Na}=3.81 ; \quad \mathrm{Na}-\mathrm{Na}=$ $3.04 ; \mathrm{Na}-\mathrm{D}=2.33 ; \mathrm{Ba}-\mathrm{D}=3.03 ; \mathrm{D}-\mathrm{D}=2.98$.

\section{$3.40 \quad K_{3} \operatorname{ReH}_{6}$}

By hydrogenation of powder mixtures of $\mathrm{KH}$ and $\mathrm{Re}$ at $850 \mathrm{~K}$ and $\mathrm{H}_{2}$ pressures of up to 3500 bar. $^{117}$ Reaction product has olive green color, is unstable in air and moisture, and decomposes under vacuum; ordered structure (Figure 40) from npd on deuteride: cubic variant of cryolite type $\left(\mathrm{K}_{2} \mathrm{NaAlF}_{6}\right)$, $F m \overline{3} m, Z=4$; Re site forming oct-[$\left[\mathrm{ReH}_{6}\right]^{3-} 16$-electron complexes with site symmetry $m \overline{3} m$; surrounded by $14 \mathrm{~K}^{+}$ forming a bicapped cuboctahedron (of which the closest $\mathrm{K}^{+}$, site $\mathrm{K} 1$, in eightfold cubic configuration); lif: $3 \mathrm{~K}^{+} \cdot\left[\mathrm{ReH}_{6}\right]^{3-}$; $\mathrm{Re}^{\mathrm{III}}, \mathrm{d}^{4}$ (consistent with experimentally measured weak temperature independent paramagnetism).

$\operatorname{Distances}(\AA): \operatorname{Re}-\mathrm{D}=1.71(6 \times) ; \operatorname{Re}-\mathrm{D}=3.73 ; \operatorname{Re}-\mathrm{Re}=$ $6.10 ; \mathrm{K}-\mathrm{D}=2.60 ; \mathrm{K}-\mathrm{K}=3.73 ; \mathrm{D}-\mathrm{D}=2.41$.

\section{$3.41 \mathrm{Ca}_{8} \mathrm{Rh}_{6} \mathrm{H}_{24}$}

From calcium hydride and rhodium metal by sintering powder mixtures at temperatures of up to $1133 \mathrm{~K}$ under 


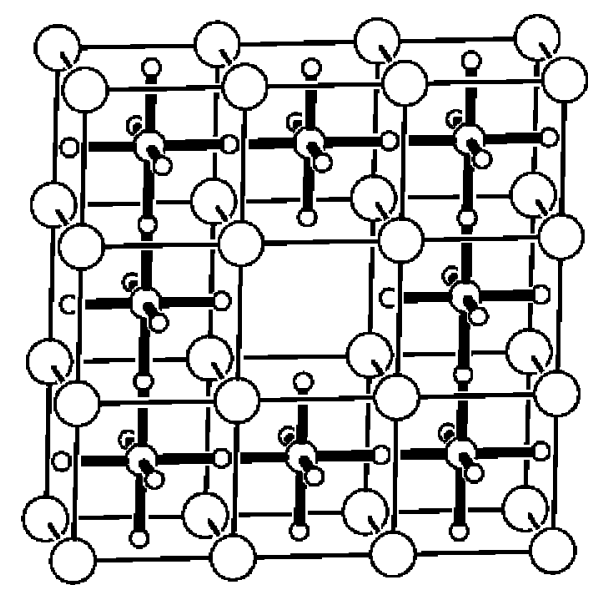

Figure 41 Structure of $\mathrm{Ca}_{8} \mathrm{Rh}_{6} \mathrm{D}_{24}$

5 bar hydrogen pressure ${ }^{93}$ powder of metallic brass-like appearance, sensitive to moisture and air; ordered structure (Figure 41) closely related to partially disordered more Rh poor $\mathrm{Ca}_{8} \mathrm{Rh}_{5} \mathrm{H}_{23}$ (XXVI), intermediate to anion deficient $\mathrm{K}_{2} \mathrm{PtCl}_{6}$ type (II) and perovskite type; from npd on deuteride: $\operatorname{Im} \overline{3} m, Z=1$; three-dimensional network of $\mathrm{Rh}$ centered, corner-sharing D octahedra (site symmetry $4 / \mathrm{mm} . \mathrm{m}$ ) consisting of four bridging (D2) and two terminal (D1); Ca surrounds octahedra in eightfold cubic configuration; lif: ${ }_{4 n} \mathrm{Ca}^{2+} \cdot\left[\mathrm{Rh}_{3} \mathrm{H}_{12}\right]_{n}{ }^{8 n-}$, mixed valence $\mathrm{Rh}^{\text {'1.33' }}$

Distances $(\AA): \mathrm{Rh}-\mathrm{D}=1.73(2 \times)$ and $1.82(4 \times) ; \mathrm{Rh}-\mathrm{Ca}=$ 3.15; Rh-Rh = 3.64; $\mathrm{Ca}-\mathrm{D}=2.58 ; \mathrm{Ca}-\mathrm{Ca}=3.64 ; \mathrm{D}-\mathrm{D}=$ 2.51 .

\section{$3.42 \mathrm{Rb}_{3} \operatorname{ReH}_{10}\left(\mathrm{~K}_{3} \operatorname{ReH}_{10}, \mathrm{Cs}_{3} \operatorname{ReH}_{10}\right)$}

By hydrogenation of powder mixtures of alkali hydrides and $\mathrm{Re}$ up to $870 \mathrm{~K}$ and $\mathrm{H}_{2}$ pressures of up to $3500 \mathrm{bar}$ $\left(\mathrm{K},{ }^{9,118} \mathrm{Rb}^{118}{ }^{118} \mathrm{Cs}^{119}\right)$; reaction products are light grey and unstable in air and moisture; disordered structure (Figure 42) from npd on $\mathrm{Rb}$ deuteride: cubic room temperature variant:

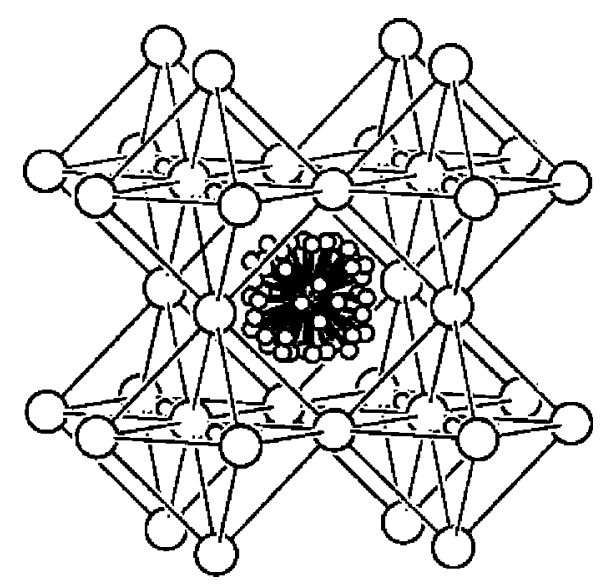

Figure 42 Structure of $\mathrm{Rb}_{3} \operatorname{ReD}_{10}$
$\operatorname{Pm} \overline{3} m, Z=1$; described as $\mathrm{ReO}_{3}$ type framework containing strongly disordered $\left[\mathrm{ReH}_{9}\right]^{2-}$ 18-electron complexes (average site symmetry $m \overline{3} m$ ) with two 24 -fold disordered D sites (occupancies 0.187 and 0.194 ), and hydride anions $\mathrm{H}^{-}$in octahedral $\mathrm{M}^{+}$environment; transforms below $110 \mathrm{~K}$ into presumably ordered ttp- $\left[\mathrm{ReH}_{9}\right]^{2-}$ complexes (described as 'monocapped square antiprisms ${ }^{\mathbf{9 , 1 1 8}}$ ) having rhombohedral symmetry; lif: $3 \mathrm{M}^{+} \cdot\left[\mathrm{ReH}_{9}\right]^{2-} \cdot \mathrm{H}^{-} ; \mathrm{Re}^{\mathrm{VII}}, \mathrm{d}^{0}$ (consistent with experimentally measured diamagnetism on $\mathrm{Rb}$ compound).

Distances for disordered structure $(\AA)$ : $R e-D=1.70(24 \times$, dis) and $1.71(24 \times$, disordered $)$, $\mathrm{Re}-\mathrm{Rb}=4.18 ; \mathrm{Rb}-\mathrm{Rb}=$ 4.18; $\mathrm{Re}-\mathrm{Re}=6.10 ; \mathrm{Rb}-\mathrm{D}=2.83 ; \mathrm{Rb}-\mathrm{D}^{-}=2.96$; $\mathrm{D}-\mathrm{D}=$ 0.58 (dis).

\subsection{3 $\mathrm{Na}_{3} \mathrm{OsH}_{7}\left(\mathrm{Na}_{3} \mathrm{RuH}_{7}\right)$}

By sintering powder mixtures of $\mathrm{NaH}$ and $\mathrm{Os}(\mathrm{Ru})$ at temperatures of up to $870 \mathrm{~K}$ and $\mathrm{H}_{2}$ pressures of up to 1500 (Os) and $6000(\mathrm{Ru})$ bar. $^{\mathbf{1 2 0}}$ ordered structure (Figure 43) by npd on deuteride of Os compounds at room temperature: $P 4_{2} /$ mnm, $Z=4$; contains isolated distorted pentagonal bipyramid $\left[\mathrm{OsH}_{7}\right]^{3-}$ 18-electron units having Os site symmetry $m .2 m$ surrounded by eight $\mathrm{Na}^{+}$(from two $\mathrm{Na}$ sites) forming a distorted cube; four D sites; transforms into a HT modification at $459 \mathrm{~K}$; lif: $3 \mathrm{Na}^{+} \cdot\left[\mathrm{TH}_{7}\right]^{3-}, \mathrm{T}^{\mathrm{IV}}, \mathrm{d}^{4}$ (supported by experimentally measured weak temperature independent paramagnetism on Os compound). Diffraction evidence ${ }^{\mathbf{1 2 0}}$ for the existence of cubic phases $\mathrm{M}_{3-\delta} \mathrm{TH}_{7-\delta}(\mathrm{M}=\mathrm{K}, \mathrm{Rb}, \mathrm{Cs}$; $\mathrm{T}=\mathrm{Ru}, \mathrm{Os} ; \delta \sim 0.12$ for the $\mathrm{K}-\mathrm{Ru}$ compound).

Distances $(\AA): \quad$ Os-D $=1.62-1.70 ; \quad \mathrm{Na}-\mathrm{Os}=3.15$; Os-Os $=5.35 ; \mathrm{Na}-\mathrm{D}=2.21 ; \mathrm{Na}-\mathrm{Na}=3.12 ; \mathrm{D}-\mathrm{D}=1.84$.

\subsection{4 $\mathrm{Cs}_{3} \mathrm{OsH}_{9}\left(\mathrm{Rb}_{3} \mathrm{OsH}_{9}\right)$}

By sintering powder mixtures of alkali hydrides and Os under conditions similar to $\mathrm{Na}_{3} \mathrm{OsH}_{7}$ (XLIII). ${ }^{120}$ Partially disordered structure (Figure 44) by npd on deuteride of Cs compound at room temperature: $\operatorname{Pm} \overline{3} m, Z=1$; contains

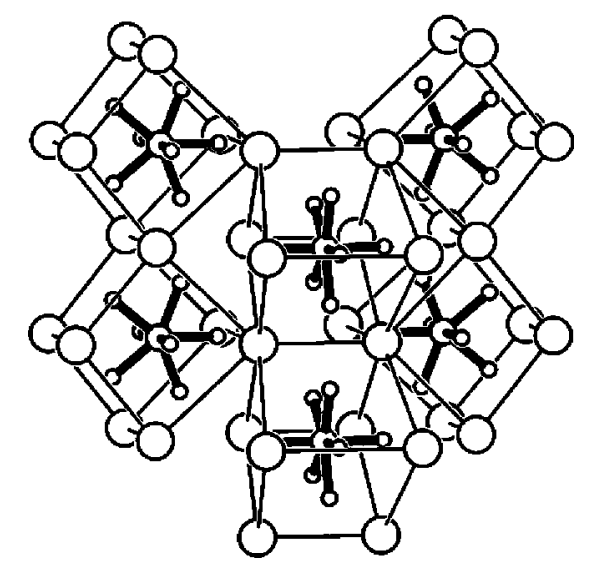

Figure 43 Structure of $\mathrm{Na}_{3} \mathrm{OsD}_{7}$ 


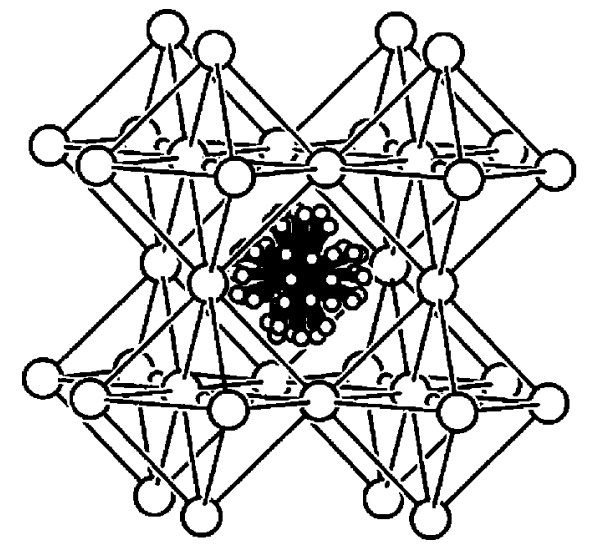

Figure 44 Structure of $\mathrm{Cs}_{3} \mathrm{OsD}_{9}$

isolated $\left[\mathrm{OsH}_{8}\right]^{3-}$ 18-electron units having strongly disordered $\mathrm{D}$ ligands at room temperature as described by two partially occupied 24-fold D sites (occupancy $=1 / 6$, not refined), and one $\mathrm{D}^{-}$site in octahedral $\mathrm{M}^{+}$surrounding; Os (average site symmetry $m \overline{3} m$ ) surrounded by eight $\mathrm{M}^{+}$in cubic configuration; lif: $3 \mathrm{M}^{+} \cdot\left[\mathrm{OsH}_{8}\right]_{\mathrm{av}}{ }^{2-} \cdot \mathrm{H}^{-} ; \mathrm{Os}^{\mathrm{VI}}, \mathrm{d}^{2}$.

Distances $(\AA)$ : Os-D = $1.59(24 \times$, dis $)-1.70(24 \times$, dis $)$; Os-Cs $=4.33$; Os-Os $=6.13 ; \mathrm{Cs}-\mathrm{D}=2.91 ; \mathrm{Cs}^{-} \mathrm{D}^{-}=3.06$; $\mathrm{Cs}-\mathrm{Cs}=4.33 ; \mathrm{D}-\mathrm{D}=0.70$ (dis).

\section{$3.45 \mathrm{Mg}_{6} \mathrm{Ir}_{2} \mathrm{H}_{11}$}

From either hydrogenated binary $\mathrm{Mg}_{3} \mathrm{Ir}$ alloy or by sintering powder mixtures of the elements at up to $773 \mathrm{~K}$ under a $\mathrm{H}_{2}$ pressure of up to $150 \mathrm{bar}^{121}$ Red colored hydride; partially disordered structure (Figure 45) from npd on deuteride, $P 2_{1} / c, Z=8$; contains four symmetry independent Ir hydride complexes: a spy $\left[\mathrm{IrH}_{5}\right]^{4-}$ and three sad $\left[\mathrm{IrH}_{4}\right]^{5-}$ complexes of which two are disordered, and five hydride anions are exclusively bonded to $\mathrm{Mg}$; all complexes are surrounded by $\mathrm{Mg}$ in distorted cubic

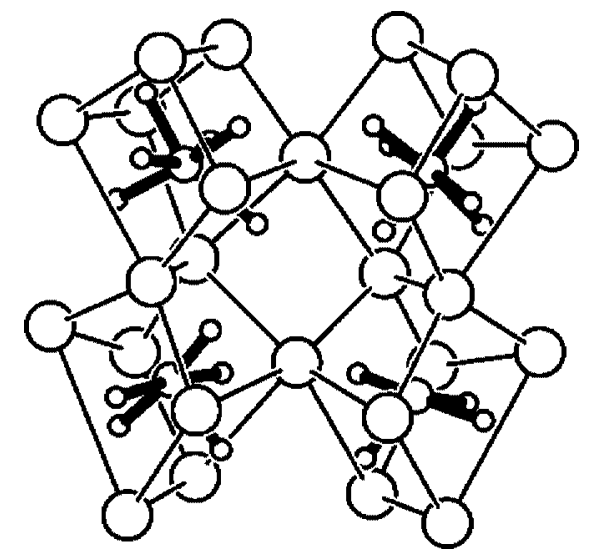

Figure 45 Structure of $\mathrm{Mg}_{6} \mathrm{Ir}_{2} \mathrm{D}_{11}$ configurations; metal substructure represents a monoclinic distortion of orthorhombic Co analog $\mathrm{Mg}_{6} \mathrm{Co}_{2} \mathrm{D}_{11}$ (XVII); lif: $12 \mathrm{Mg}^{2+} \cdot\left[\mathrm{IrH}_{5}\right]^{4-} \cdot\left[\mathrm{IrH}_{4}\right]^{5-} \cdot 2\left[\mathrm{IrH}_{4}\right]_{\mathrm{av}}{ }^{5-} \cdot 5 \mathrm{H}^{-}$; mixed valence $\mathrm{Ir}^{\mathrm{I}}, \mathrm{d}^{8}$ and $\mathrm{Ir}^{-I}, \mathrm{~d}^{10}$.

Distances $(\AA)$ : $\operatorname{Ir} 1-\mathrm{D}=1.67-2.07, \operatorname{Ir} 2-\mathrm{D}=1.66-1.74$, Ir $1 \mathrm{a}-\mathrm{D}=1.67-1.99$, Ir $2 \mathrm{a}-\mathrm{D}=1.64-1.90 ; \mathrm{Ir}-\mathrm{Mg}=2.65$; $\mathrm{Ir}-\mathrm{Ir}=4.62 ; \mathrm{Mg}-\mathrm{Mg}=2.79 ; \mathrm{Mg}-\mathrm{D}=1.79 ; \quad \mathrm{Mg}-\mathrm{D}^{-}=$ $1.74 ; \mathrm{D}-\mathrm{D}=2.11$.

\subsection{6 $\mathrm{NdMgNi}_{4} \mathrm{H}_{3.6}\left(\mathrm{LaMgNi}_{4} \mathrm{H} \sim_{3.6}\right)$}

From hydrogenation of intermetallic compounds $\mathrm{NdMgNi}_{4}$ and $\mathrm{LaMgNi}_{4}$ at $323 \mathrm{~K}$ under $\mathrm{H}_{2}$ pressure of up to 7-8 bar; ${ }^{\mathbf{1 2 2}}$ metallic powders decompose in air by catalytic water formation; nearly ordered structure of $\mathrm{NdMgNi}_{4} \mathrm{H}_{3.6}$ (Figure 46) from npd on deuteride; $P n m 2_{1}, Z=4$; contains three $\mathrm{Ni}$ sites and three almost fully occupied $\mathrm{D}$ sites (occupancies $0.89-0.92$ ) forming $\left[\mathrm{Ni}_{4} \mathrm{H}_{\sim 4}\right]^{5-}$ tetramers in which three edges and one face of the Ni tetrahedron is bridged by hydrogen; tetramers are surrounded by monocapped trigonal prisms formed by four $\mathrm{Nd}$ and three $\mathrm{Mg}$; lif: $\mathrm{M}^{3+} \cdot \mathrm{Mg}^{2+} \cdot\left[\mathrm{Ni}_{4} \mathrm{H}_{\sim 4}\right]^{5-}$.

Distances $\quad(\AA): \quad \mathrm{Ni}-\mathrm{D}=1.64-1.72 ; \quad \mathrm{Ni}-\mathrm{Ni}=2.44$; $\mathrm{Ni}-\mathrm{Mg}=2.83 ; \mathrm{Ni}-\mathrm{Nd}=2.90 ; \mathrm{Mg}-\mathrm{Mg}=4.92 ; \mathrm{Mg}-\mathrm{Nd}=$ 3.14; $\mathrm{Mg}-\mathrm{D}=2.17 ; \mathrm{Nd}-\mathrm{Nd}=4.98 ; \mathrm{Nd}-\mathrm{D}=2.34 ; \mathrm{D}-\mathrm{D}=$ 2.47 .

\subsection{7 $\mathrm{LaMg}_{2} \mathrm{NiH}_{7}$}

From hydrogenation of ternary metal compound $\mathrm{LaMg}_{2} \mathrm{Ni}$ below $473 \mathrm{~K}$ under $\mathrm{H}_{2}$ pressure of up to 8 bar; ${ }^{123}$ dark grey colored powder sable in air; ordered structure (Figure 47) from npd on deuteride: $P 2_{1} / c, Z=8$; contains two symmetry independent tet- $\left[\mathrm{NiH}_{4}\right]^{4-}$ 18-electron complexes having eight terminal $\mathrm{D}$ atoms, and six hydride anions bonded to two $\mathrm{Mg}$ and two $\mathrm{La}$ only; $\mathrm{Ni}$ (site symmetry 1) surrounded by three $\mathrm{La}$ (two sites) and six $\mathrm{Mg}$ (four sites) in monocapped square antiprismatic configuration;

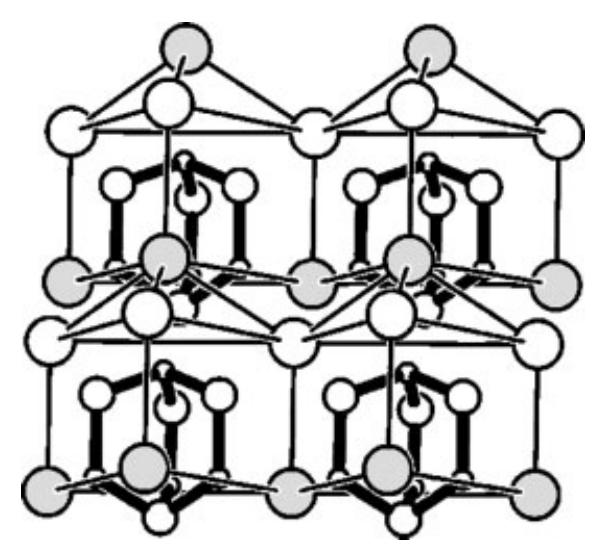

Figure 46 Structure of $\mathrm{NdMgNi}_{4} \mathrm{D}_{\sim 4}$ 


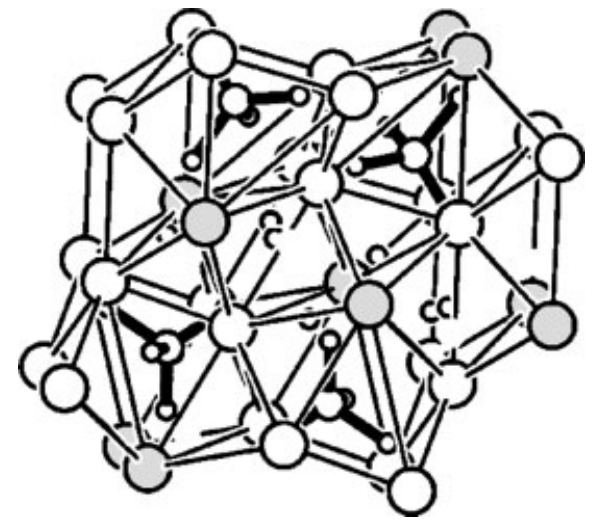

Figure 47 Structure of $\mathrm{LaMg}_{2} \mathrm{NiD}_{7}$

lif: $\mathrm{La}^{3+} \cdot 2 \mathrm{Mg}^{2+} \cdot\left[\mathrm{NiH}_{4}\right]^{4-} \cdot 3 \mathrm{H}^{-} ; \mathrm{Ni}^{0}, \mathrm{~d}^{10}$; hydride is nonmetallic; provides example for hydrogation induced metal $\left(\mathrm{LaMg}_{2} \mathrm{Ni}\right)$ - to - nonmetal $\left(\mathrm{LaMg}_{2} \mathrm{NiH}_{7}\right)$ transition.

Distances $(\AA)$ : Ni1-D = 1.49-1.63, Ni2-D = 1.48-1.63; $\mathrm{Ni}-\mathrm{Mg}=2.59 ; \mathrm{Ni}-\mathrm{La}=3.36 ; \mathrm{Ni}-\mathrm{Ni}=4.14 ; \mathrm{Mg}-\mathrm{Mg}=$ 3.02; $\quad \mathrm{Mg}-\mathrm{La}=3.42 ; \quad \mathrm{La}-\mathrm{La}=4.06 ; \quad \mathrm{Mg}-\mathrm{D}=1.94$; $\mathrm{Mg}-\mathrm{D}^{-}=1.82 ; \mathrm{La}-\mathrm{D}=2.39 ; \mathrm{La}-\mathrm{D}^{-}=2.33 ; \mathrm{D}-\mathrm{D}=2.12$.

\section{CRYSTAL CHEMISTRY}

\subsection{Hydride Complexes}

Occurrence. At present, some 50 different homoleptic hydride complexes have been identified in over 127 compounds that cover 47 structure types. Their occurrence across the $3 \mathrm{~d}, 4 \mathrm{~d}$, and $5 \mathrm{~d}$ transition metal series is summarized in Table 19. The complexes form with transition elements from group seven $(\mathrm{Mn})$, eight $(\mathrm{Fe})$, nine $(\mathrm{Co})$ and ten $(\mathrm{Ni})$, to closed d-shell elements of group eleven $(\mathrm{Cu})$ and twelve ( $\mathrm{Zn})$. Hydride complexes have not been characterized as yet for elements of group four (Ti), five (V) and six (Cr), and none for $\mathrm{Ag}, \mathrm{Au}$, and $\mathrm{Hg}$. Interestingly, most complexes contain T-metals that do not form stable binary hydrides such as iron, cobalt, and congeners, or form relatively unstable hydrides such as nickel and congeners. While the great majority of complexes are centred by one T-metal atom ('mononuclear' complexes) and display terminal hydrogen ligands only, some are centred by two $(\mathrm{Ru}, \mathrm{Pt})$, four $(\mathrm{Rh}, \mathrm{Ni})$ or more $(\mathrm{Ru}, \mathrm{Rh})$ and display both terminal and bridging hydrogen ligands, and/or T-T metal-metal bonds ('polynuclear' complexes).

Mononuclear Complexes. They show at least 10 different geometries that are represented in Table 20. They range from tricapped trigonal prismatic (e.g. $\left[\mathrm{ReH}_{9}\right]^{2-}$ ), pentagonal bipyramidal (e.g. $\left[\mathrm{OsH}_{7}\right]^{3-}$ ), octahedral (e.g.
$\left.\left[\mathrm{FeH}_{6}\right]^{4-},\left[\mathrm{ReH}_{6}\right]^{3-}\right)$, square pyramidal (e.g. $\left[\mathrm{CoH}_{5}\right]^{4-}$ ), planar (e.g. $\left.\left[\mathrm{RhH}_{4}\right]^{3-}\right)$, tetrahedral (e.g. $\left.\left[\mathrm{NiH}_{4}\right]^{4-}\right)$, saddle-like (e.g. $\left[\mathrm{IrH}_{4}\right]^{5-}$ ), triangular (e.g. $\left.\left[\mathrm{PdH}_{3}\right]^{3-}\right)$, T-shaped (e.g. $\left[\mathrm{RuH}_{3}\right]^{6-}$ ) to linear (e.g. $\left[\mathrm{PdH}_{2}\right]^{2-}$ ). Generally speaking, the ligand geometries resemble those of transition metals in 'inorganic molecules ${ }^{\mathbf{1 2 4}}$ ' and coordination compounds such as metal carbonyls and cyanides, and are consistent with conventional electron counts, that is, they are octahedral (usually 18 electrons and $\mathrm{d}^{6}$ ), such as $\mathrm{Mo}(\mathrm{CO})_{6}$; square planar (usually 16 electrons, $\left.\mathrm{d}^{8}\right)$, such as $\mathrm{Ni}(\mathrm{CN})_{4}{ }^{2-}$; square pyramidal $\left(18\right.$ electrons, $\left.\mathrm{d}^{8}\right)$, such as $\left[\mathrm{Ni}(\mathrm{CN})_{5}\right]^{3-}$; saddle-like, such as the binuclear nonbridged structure of $\mathrm{Co}_{2}(\mathrm{CO})_{8}$ (formally 17 electrons, $\left.\mathrm{d}^{9}\right)$ or the trinuclear structure of $\mathrm{Ru}_{3}(\mathrm{CO})_{12}$ (formally 16 electrons, $\mathrm{d}^{8}$ ); tetrahedral (usually 18 electrons, $\left.\mathrm{d}^{10}\right)$, such as $\mathrm{Ni}(\mathrm{CO})_{4}$; or linear (usually 14 electrons, $\mathrm{d}^{10}$ ), such as the usual ligand geometry of $\mathrm{Ag}^{\mathrm{I}}\left(\mathrm{d}^{10}\right)$ and congeners. Complexes having less than 13 electrons or more than 18 electrons have not yet been reported. The tetrahedral 13-electron $\left[\mathrm{MnH}_{4}\right]^{2-}$ complex in $\mathrm{K}_{3} \mathrm{MnH}_{5}(\mathrm{XXX})$ is remarkable because it displays a half-filled $\mathrm{d}$ shell and leads to a pink colored hydride that orders magnetically. Other interesting cases are the T-shaped formally 17 -electron $\left[\mathrm{RuH}_{3}\right]^{6-}$ complex in $\mathrm{Mg}_{3} \mathrm{RuH}_{3}(\mathrm{XV})$ and the saddle-like formally 16electron $\left[\mathrm{RuH}_{4}\right]^{4-}$ complex in $\mathrm{Mg}_{2} \mathrm{RuH}_{4}$ (XVIII), because they provides possible links to polynuclear complexes (see below). Some complex geometries represent only averages such as $\left[\mathrm{CoH}_{4}\right]_{\mathrm{av}}{ }^{5-},\left[\mathrm{RuH}_{5}\right]_{\mathrm{av}}{ }^{5-},\left[\mathrm{OsH}_{8}\right]_{\mathrm{av}}{ }^{2-}$ (not included in Table 20), $\left[\mathrm{PdH}_{3}\right]_{\mathrm{av}}{ }^{3-}$, and $\left[\mathrm{IrH}_{3}\right]_{\mathrm{av}}{ }^{6-}$ (not included in Table 20), because of structural disorder. A five-coordinate trigonal bipyramidal hydrogen configuration similar to the ligand geometry in $\mathrm{Fe}(\mathrm{CO})_{5}$ has not yet been reported. The presumably square antiprismatic configuration in $\mathrm{Cs}_{3} \mathrm{OsH}_{9}$ (XLIV) has not yet been ascertained.

Some T-metals adopt one type of ligand geometry only, such as $\mathrm{Fe}$ that forms exclusively oct- $\left[\mathrm{FeH}_{6}\right]^{4-}$ complexes (occurring in three hydride structure types, see Table 19), while others adopt two geometries, such as Co that forms sad- $\left[\mathrm{CoH}_{4}\right]^{5-}$ and spy- $\left[\mathrm{CoH}_{5}\right]^{4-}$ complexes (either ordered or disordered), or three or more geometries, such as Ru that forms five: ts- $\left[\mathrm{RuH}_{3}\right]^{6-}$, sad-[ $\left[\mathrm{RuH}_{4}\right]^{4-}$, spy-[RuH$]_{\mathrm{av}}{ }^{4-}$, oct$\left[\mathrm{RuH}_{6}\right]^{4-}$, and pbp-[RuH$]_{7}^{3-}$. Each hydride structure usually contains one crystallographic T-metal site. Exceptions are $\mathrm{K}_{2} \mathrm{ReH}_{9}$ (I), $\mathrm{Mg}_{6} \mathrm{Co}_{2} \mathrm{H}_{11}$ (XVII), and $\mathrm{LaMg}_{2} \mathrm{NiH}_{7}$ (XLVII) that contain two, $\mathrm{K}_{3} \mathrm{PdH}_{3}$ (IX), $\mathrm{Sr}_{8} \mathrm{Rh}_{5} \mathrm{H}_{23}$ (XXVI), and $\mathrm{LaMgNi}_{4} \mathrm{H}_{4}$ (XLVI) that contain three, and $\mathrm{Mg}_{6} \mathrm{Ir}_{2} \mathrm{H}_{11}$ (XLV) that contains four independent $\mathrm{T}$-metal sites. Among these $\mathrm{Mg}_{6} \mathrm{Co}_{2} \mathrm{H}_{11}$ (XVII) and $\mathrm{Mg}_{6} \mathrm{Ir}_{2} \mathrm{H}_{11}$ (XLV) are remarkable because the T-metal sites display different complex geometries in the same structure: one sad- $\left[\mathrm{TH}_{4}\right]^{5-}$ and three spy$\left[\mathrm{TH}_{5}\right]^{4-}$ of which two are disordered $(\mathrm{T}=\mathrm{Co}, \mathrm{Ir})$. T-metal complexes having a given geometry usually occurs in different structure types such as oct- $\left[\mathrm{FeH}_{6}\right]^{4-}$ in $\mathrm{Mg}_{2} \mathrm{FeH}_{6}$ (II), $\mathrm{SrMg}_{2} \mathrm{FeH}_{6}$ (XVI) and $\mathrm{Ca}_{4} \mathrm{Mg}_{4} \mathrm{Fe}_{3} \mathrm{H}_{22}$ (XX), and tet$\left[\mathrm{NiH}_{4}\right]^{4-}$ in $\mathrm{Mg}_{2} \mathrm{NiH}_{4}$ (V) $\mathrm{CaMgNiH}_{4}$ (XIX) and $\mathrm{LaMg}_{2} \mathrm{NiH}_{7}$ (XLVII). Complexes centred by different $\mathrm{T}$-metals in the 
Table 19 Homonuclear and polynuclear complex anions as found in various hydride structure types

\begin{tabular}{|c|c|c|c|c|c|c|c|c|}
\hline Mn & \multicolumn{2}{|c|}{$\mathbf{F e}$} & \multicolumn{2}{|l|}{ Co } & \multicolumn{2}{|c|}{$\mathbf{N i}$} & $\mathrm{Cu}$ & $\mathbf{Z n}$ \\
\hline $\begin{array}{ll}{\left[\mathrm{MnH}_{4}\right]^{2-}} & \text { XXX } \\
{\left[\mathrm{MnH}_{6}\right]^{5-}} & \text { XXIII }\end{array}$ & {$\left[\mathrm{FeH}_{6}\right]^{4-}$} & II, XVI, XX & $\begin{array}{l}{\left[\mathrm{CoH}_{4}\right]^{5-},} \\
{\left[\mathrm{CoH}_{4}\right]_{\mathrm{av}}^{5-}} \\
{\left[\mathrm{CoH}_{5}\right]^{4-}} \\
{\left[\mathrm{CoH}_{5}\right]_{\mathrm{av}}{ }^{4-}}\end{array}$ & $\begin{array}{l}\text { XVII } \\
\text { III } \\
\text { XVII, } \\
\text { XXXII }\end{array}$ & $\begin{array}{l}{\left[\mathrm{NiH}_{4}\right]^{4-}} \\
{\left[\mathrm{Ni}_{4} \mathrm{H}_{4}\right]^{5-}} \\
{\left[\mathrm{NiH}_{3-x}\right]_{\mathrm{n}, \mathrm{av}}^{2 n-}}\end{array}$ & $\begin{array}{l}\text { V, XIX, } \\
\text { XLVII } \\
\text { XLVI } \\
\text { X }\end{array}$ & {$\left[\mathrm{CuH}_{4}\right]^{3-} \mathrm{XXXV}$} & $\begin{aligned} & {\left[\mathrm{ZnH}_{4}\right]^{2-} } \mathrm{XXIX} \\
& \mathrm{XXX}\end{aligned}$ \\
\hline Tc & \multicolumn{2}{|c|}{$\mathbf{R u}$} & \multicolumn{2}{|l|}{$\mathbf{R h}$} & \multicolumn{2}{|c|}{ Pd } & Ag & Cd \\
\hline$\left[\begin{array}{ll}\left.\mathrm{TcH}_{9}\right]^{2-} & \mathrm{I}\end{array}\right.$ & $\begin{array}{l}{\left[\mathrm{Ru}_{2} \mathrm{H}_{6}\right]^{12-}} \\
{\left[\mathrm{RuH}_{4}\right]_{n}{ }^{4 n-}} \\
{\left[\mathrm{RuH}_{5}\right]_{\mathrm{av}}^{5-}} \\
{\left[\mathrm{RuH}_{6}\right]^{{ }^{-}}} \\
\\
{\left[\mathrm{RuH}_{7}\right]^{3-}}\end{array}$ & $\begin{array}{l}\text { XV } \\
\text { XVIII } \\
\text { XXV } \\
\text { II, XI, } \\
\text { XXVII, } \\
\text { XXXVI, } \\
\text { XXXVIII } \\
\text { XLIII }\end{array}$ & $\begin{array}{l}{\left[\mathrm{Rh}_{4} \mathrm{H}_{4}\right]^{8-}} \\
{\left[\mathrm{RhH}_{4}\right]^{3-}} \\
{\left[\mathrm{Rh}_{3} \mathrm{H}_{12}\right]_{n}{ }^{8 n-}} \\
{\left[\mathrm{Rh}_{4} \mathrm{H}_{17}\right]_{n}{ }_{n}^{13 n-}} \\
{\left[\mathrm{RhH}_{5}\right]_{{ }_{3 v}-}} \\
{\left[\mathrm{RhH}_{6}\right]^{3-}}\end{array}$ & $\begin{array}{l}\text { XIV } \\
\text { XIII } \\
\text { XLI } \\
\text { XXVI } \\
\text { III } \\
\text { XII, } \\
\text { XXVI }\end{array}$ & $\begin{array}{l}{\left[\mathrm{PdH}_{2}\right]^{2-}} \\
{\left[\mathrm{PdH}_{2+x}\right]_{n, \mathrm{av}}^{2 n-}} \\
{\left[\mathrm{PdH}_{3}\right]^{3-}} \\
{\left[\mathrm{PdH}_{3}\right]_{\mathrm{av}}{ }^{3-}} \\
{\left[\mathrm{PdH}_{4}\right]^{2-}} \\
\left.\mathrm{PdH}_{4}\right]^{4-}\end{array}$ & $\begin{array}{l}\text { VII, IX } \\
\text { X } \\
\text { XXXIX } \\
\text { XXII } \\
\text { IV, VI, } \\
\text { VIII } \\
\text { XXIX }\end{array}$ & & {$\left[\mathrm{CdH}_{4}\right]^{2-} \mathrm{XXX}$} \\
\hline $\operatorname{Re}$ & \multicolumn{2}{|c|}{ Os } & \multicolumn{2}{|l|}{ Ir } & \multicolumn{2}{|c|}{$\mathbf{P t}$} & Au & Hg \\
\hline $\begin{array}{ll}\left.\mathrm{ReH}_{6}\right]^{3-} & \text { XL } \\
{\left[^{5 e H}\right]^{5-}} & \text { XXIII } \\
{\left[\mathrm{ReH}_{9}\right]^{2-}} & \text { I, } \\
& \text { XXVIII, } \\
& \text { XXXIII } \\
{\left[\mathrm{ReH}_{9}\right]_{\mathrm{av}}{ }^{2-}} & \text { XLII }\end{array}$ & $\begin{array}{l}{\left[\mathrm{OsH}_{7}\right]^{3-}} \\
{\left[\mathrm{OsH}_{8}\right]_{\mathrm{av}}{ }^{2-}}\end{array}$ & $\begin{array}{l}\text { II, XI, } \\
\text { XXVII, } \\
\text { XXXVI, } \\
\text { XXXVIII } \\
\text { XLIII } \\
\text { XLIV }\end{array}$ & $\begin{array}{l}{\left[\mathrm{IrH}_{3}\right]_{\mathrm{av}}{ }^{6-}} \\
{\left[\mathrm{IrH}_{4}\right]^{5-}} \\
{\left[\mathrm{IrH}_{4}\right]_{\mathrm{av}}{ }^{5-}} \\
{\left[\mathrm{IrH}_{5}\right]^{4-}} \\
{\left[\mathrm{IrH}_{5}\right]_{\mathrm{av}}{ }^{4-}} \\
{\left[\mathrm{IrH}_{6}\right]^{3-}}\end{array}$ & $\begin{array}{l}\text { XXIV } \\
\text { XLV } \\
\text { XLV } \\
\text { XLV } \\
\text { III } \\
\text { XII, } \\
\text { XXXI }\end{array}$ & $\begin{array}{l}{\left[\mathrm{PtH}_{2}\right]^{2-}} \\
{\left[\mathrm{PtH}_{4}\right]^{2-}} \\
\\
{\left[\mathrm{Pt}_{2} \mathrm{H}_{9}\right]^{5-}} \\
{\left[\mathrm{PtH}_{6}\right]^{2-}}\end{array}$ & $\begin{array}{l}\text { XXXVII } \\
\text { IV, VI, } \\
\text { VIII, XXI } \\
\text { XXXIV } \\
\text { II }\end{array}$ & & \\
\hline
\end{tabular}

same structure have not yet been reported. 'Composite' hydride structures made up by covalent bonded hydride complexes and ionic bonded hydrogen occur with $\mathrm{SrMg}_{2} \mathrm{FeH}_{8}$ (XVI), $\mathrm{Ba}_{2} \mathrm{PtH}_{6}$ (XXI), $\mathrm{LiSr}_{2} \mathrm{PdH}_{5}$ (XXII), $\mathrm{Mg}_{3} \mathrm{ReH}_{7}$ (XXIII), $\mathrm{Mg}_{3} \mathrm{RuH}_{6} \quad$ (XXV), $\mathrm{LiMg}_{2} \mathrm{RuH}_{7} \quad$ (XXVII), $\mathrm{LiMg}_{4} \mathrm{Os}_{2} \mathrm{H}_{13}$ (XXXVI), $\mathrm{LaMg}_{2} \mathrm{NiH}_{7}$ (XLVII), and can be studied on Figures 16, 21, 22, 23, 25, 27, 36, and 47, respectively. Those belonging to the series $\mathrm{LiH} \cdot n \mathrm{Mg}_{2} \mathrm{TH}_{4}(\mathrm{~T}=\mathrm{Ru}$, Os; $n=1,2, \infty$, groups XXVII, XXXVI, and II, respectively) are made up by slabs of $\left[\mathrm{TH}_{6}\right]^{4-}$ complexes and sheets of ionic $\mathrm{Li}^{+} \mathrm{H}^{-}$. Finally, only relatively few $3 d$ analogs exist to the more numerous $4 \mathrm{~d}$ and $5 \mathrm{~d}$ hydrides, that is, four $\mathrm{Mn}$ versus eight $\mathrm{Re}$ compounds, five Co versus twelve $\mathrm{Rh}$ and ten $\mathrm{Ir}$ compounds, and ten $\mathrm{Ni}$ versus twenty-one $\mathrm{Pd}$ and seventeen Pt compounds. In particular, no iron analog exists for the ruthenium (osmium) series of composite structures, and no nickel analogs exist for any of the palladium and platinum compounds.

Polynuclear Complexes. Dinuclear complexes occur in nonmetallic $\mathrm{Li}_{5} \mathrm{Pt}_{2} \mathrm{H}_{9}$ (XXXIV) and metallic $\mathrm{Mg}_{3} \mathrm{RuH}_{3}$ (XV). The former displays planar $\mathrm{PtH}_{4}$ units that are connected via $\mathrm{Pt}-\mathrm{H}-\mathrm{Pt}$ bridges to $\left[\mathrm{Pt}_{2} \mathrm{H}_{9}\right]^{5-}$ complexes, and the latter display T-shaped $\mathrm{RuH}_{3}$ units that are possibly connected via $\mathrm{Ru}-\mathrm{Ru}$ bonds to $\left[\mathrm{Ru}_{2} \mathrm{H}_{6}\right]^{12-}$ dimers. Tetranuclear complexes occur in metallic $\mathrm{MgRhH}_{0.94}$ (XIV) and $\mathrm{NdMgNi}_{4} \mathrm{H}_{\sim 4}$ (XLVI). The former displays cyclic tetramers $\left[\mathrm{Rh}_{4} \mathrm{H}_{\sim 4}\right]^{8-}$ having nearly linear T-H-T bridges, and the latter tetrahedron shaped $\left[\mathrm{Ni}_{4} \mathrm{H}_{\sim 4}\right]^{5-}$ units in which three edges and one face of the Ni tetrahedron are bridged by hydrogen. Quasi onedimensional $\left[\mathrm{Ru}_{n} \mathrm{H}_{4 n}\right]^{4 n-}$ polymers displaying $\mathrm{T}-\mathrm{T}$ bonds can be formulated in $\mathrm{Mg}_{2} \mathrm{RuH}_{4}$ (XVIII), and a two-dimensional disordered $\left[\mathrm{Rh}_{4} \mathrm{H}_{17}\right]_{n}{ }^{13 n-}$ network of corner-sharing $\mathrm{RhH}_{6}$ octahedra occurs in $\mathrm{Ca}_{8} \mathrm{Rh}_{5} \mathrm{H}_{23}$ (XXVI). Three-dimensional ordered networks of corner-sharing $\mathrm{TH}_{6}$ octahedra occur in brass colored $\mathrm{Ca}_{8} \mathrm{Rh}_{6} \mathrm{H}_{24}$ (XLI; $\left[\mathrm{Rh}_{3} \mathrm{H}_{12}\right]_{n}{ }^{8 n-}$ anion), and the perovskite structure of metallic EuPdH $3\left(\mathrm{X} ;\left[\mathrm{PdH}_{3}\right]_{n}{ }^{2 n-}\right.$ anion). Polynuclear complexes with mixed T-metals such as in metal carbonyls have not been reported as yet.

Complex Formation In Intermetallic ('Interstitial') Hydrides. The historically first example for a hydrogenation induced complex formation is $\mathrm{Mg}_{2} \mathrm{NiH}_{4}(\mathrm{~V})$. The brownish colored hydride derives by hydrogenation of the intermetallic compound $\mathrm{Mg}_{2} \mathrm{Ni}$ and was originally classified as an interstitial' hydride. It contains an ordered array of $\left[\mathrm{NiH}_{4}\right]^{4-}$ complexes, is stoichiometric and nonmetallic, and shows a relatively important but reversible rearrangement of its metal atom substructure. Complex formation also occurs in other metal-rich metal framework structures such as the palladium compounds $\mathrm{CaPdH}_{2}$ (X and analogs) and $\mathrm{LiSr}_{2} \mathrm{PdH}_{5}$ (XXII). The hydrides have CsCl-type metal substructures and can be described in terms of isolated, partially disordered $\left[\mathrm{PdH}_{2}\right]_{\mathrm{av}}{ }^{2-}$ and $\left[\mathrm{PdH}_{3}\right]_{\mathrm{av}}{ }^{3-}$ groups, respectively, but the data are also compatible with 3-dimensional networks of $\mathrm{Pd}-\mathrm{H}-\mathrm{Pd}$ bridges as in 'interstitial' hydrides. ${ }^{\mathbf{2 3}}$ More palladium rich hydrides based on close-packed metal atom arrangements are $\mathrm{NaPd}_{3} \mathrm{H}_{2}{ }^{125}$ and $\mathrm{LiPdH}_{0.7}{ }^{126}$ (not included in this review). While the former shows an ordered array of well-defined linear $\mathrm{H}-\mathrm{Pd}-\mathrm{H}$ 
Table 20 Homonuclear transition metal hydride complexes ${ }^{a}$ and formal electron configurations

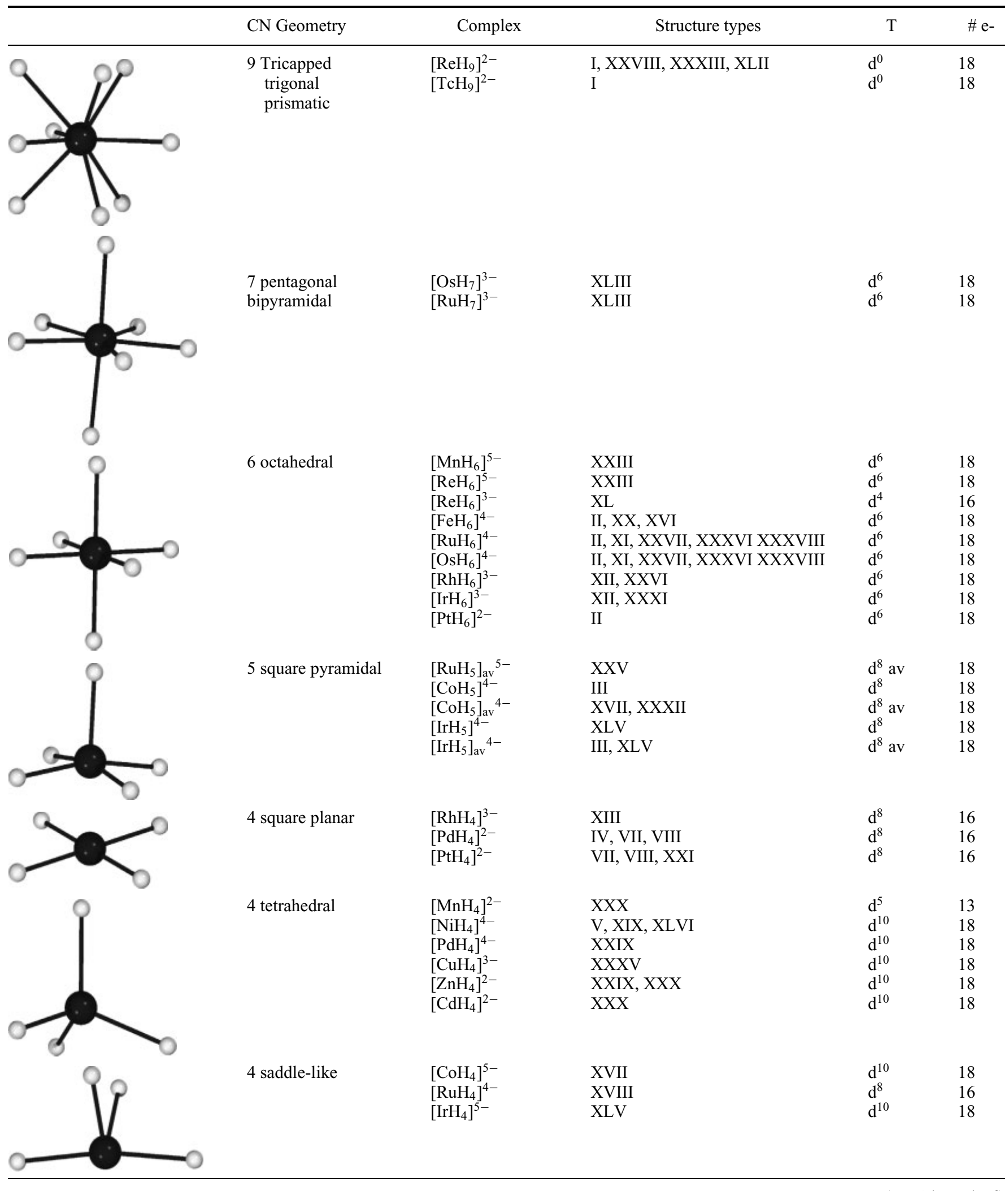


Table 20 cont'd

\begin{tabular}{|c|c|c|c|c|c|}
\hline & CN Geometry & Complex & Structure types & $\mathrm{T}$ & \# e- \\
\hline & 3 triangular & {$\left[\mathrm{PdH}_{3}\right]^{3-}$} & XXXIX & $\mathrm{d}^{10}$ & 16 \\
\hline & 3 T-shaped & $\begin{array}{l}{\left[\mathrm{RuH}_{3}\right]^{6-}} \\
{\left[\mathrm{PdH}_{3}\right]_{\mathrm{av}}{ }^{3-}}\end{array}$ & $\begin{array}{l}\text { XV } \\
\text { XXII }\end{array}$ & $\begin{array}{l}\text { Dimer } \\
d^{10} \text { av }\end{array}$ & $\begin{array}{l}17 \\
16\end{array}$ \\
\hline & 2 linear & $\begin{array}{l}{\left[\mathrm{PdH}_{2}\right]^{2-}} \\
{\left[\mathrm{PdH}_{2}\right]_{\mathrm{av}}{ }^{2-}} \\
{\left[\mathrm{PtH}_{2}\right]^{2-}}\end{array}$ & $\begin{array}{l}\text { VI, IX } \\
\text { X } \\
\text { XXXVII }\end{array}$ & $\begin{array}{l}d^{10} \\
d^{10} \text { av } \\
d^{10}\end{array}$ & $\begin{array}{l}14 \\
14 \\
14\end{array}$ \\
\hline
\end{tabular}

${ }^{\mathrm{a}}$ In decreasing coordination number, $\mathrm{CN}$; only structurally characterized, essentially ordered monomers having terminal $\mathrm{H}$ ligands are included; av: average due to disorder.

groups, the latter is disordered and shows only a weak tendency for complex formation. As to the above mentioned metallic hydrides $\mathrm{MgRhH}_{\sim 0.9}(\mathrm{XIV})$ and $\mathrm{NdMgNi}_{4} \mathrm{H}_{\sim 4}$ (XLVI), their hydrogen distributions can be described as 'interstitial', but the stereochemical activity of the tetramers $\left[\mathrm{Rh}_{4} \mathrm{H}_{4}\right]^{8-}$ and $\left[\mathrm{Ni}_{4} \mathrm{H}_{4}\right]^{5-}$, respectively, is evident. These findings suggest that hydride complex formation could be a general phenomenon in interstitial hydrides, at least on a local level. Some striking examples for hydrogenation induced complex formations and metal-insulator transitions have been recently reported in the systems $\mathrm{Mg}_{3} \mathrm{Ir}-\mathrm{H}_{2}{ }^{121}$ and $\mathrm{LaMg}_{2} \mathrm{Ni}-\mathrm{H}_{2} \cdot{ }^{123}$ The former contains the intensely red colored hydride $\mathrm{Mg}_{6} \mathrm{Ir}_{2} \mathrm{H}_{11}$ (XLV) the structure of which can be rationalized in terms of pyr$\left[\mathrm{IrH}_{5}\right]^{4-}$ and sad-[ $\left[\mathrm{IrH}_{4}\right]^{5-}$ 18-electron complexes and hydride anions $\mathrm{H}^{-}$, in agreement with the limiting ionic formula $4 \mathrm{Mg}_{6} \mathrm{Ir}_{2} \mathrm{H}_{11}=5 \mathrm{MgH}_{2} \cdot 19 \mathrm{Mg}^{2+} \cdot 2\left[\mathrm{IrH}_{5}\right]^{4-} \cdot 6\left[\mathrm{IrH}_{4}\right]^{5-}$. The latter contains the grey colored hydride $\mathrm{LaMg}_{2} \mathrm{NiH}_{7}$ (XLVII) that is nonmetallic. Its structure contains tetrahedral $\left[\mathrm{NiH}_{4}\right]^{4-}$ complexes and hydride anions $\mathrm{H}^{-}$corresponding to the limiting ionic formula $\mathrm{LaH}_{3} \cdot 2 \mathrm{Mg}^{+2} \cdot\left[\mathrm{NiH}_{4}\right]^{4}$. Thus both compounds can be considered as 'complex' hydrides for which the 'interstitial' concept fails.

Order-Disorder Transitions. The mobility of the hydrogen ligands (or of the entire complexes) at room temperature is generally high. Well-characterized order-disorder transitions within the complexes near room temperature occur in $\mathrm{Mg}_{2} \mathrm{CoH}_{5}$ (III), $\mathrm{Mg}_{2} \mathrm{NiH}_{4}$ (V), $\mathrm{K}_{3} \mathrm{PdH}_{3}$ (IX), $\mathrm{Na}_{2} \mathrm{PtH}_{4}$ (IV), $\mathrm{M}_{3} \mathrm{PtH}_{5}\left(\mathrm{M}=\mathrm{Rb}, \mathrm{Cs}\right.$; VIII), and $\mathrm{M}_{2} \mathrm{PtH}_{4}(\mathrm{M}=\mathrm{K}, \mathrm{Rb}, \mathrm{Cs}$; $\mathrm{VI})$. Complexes that are partially disordered at room temperature and do not readily order at low temperature occur in $\mathrm{CaPdH}_{2}$ (X), $\mathrm{LiSr}_{2} \mathrm{PdH}_{5}$ (XXII), $\mathrm{Mg}_{4} \mathrm{IrD}_{5}$ (XXIV), $\mathrm{Mg}_{3} \mathrm{RuH}_{6}$
$(\mathrm{XXV}), \mathrm{M}_{2} \mathrm{TH}_{5}(\mathrm{~T}=\mathrm{Rh}, \mathrm{Ir} ; \mathrm{M}=\mathrm{Mg}, \mathrm{Ca}, \mathrm{Sr}, \mathrm{Eu} ; \mathrm{III})$ and $\mathrm{Mg}_{6} \mathrm{~T}_{2} \mathrm{H}_{11}(\mathrm{~T}=\mathrm{Co}$; XVII, and Ir; XLV). In some structures, the hydride complexes undergo rigid motions, such as in $\mathrm{K}_{2} \mathrm{PtH}_{4}$ for which LT-NMR data suggest rotational jumps of the spl-PtH ${ }_{4}$ units in the plane of the square. ${ }^{8}$

\subsection{Cation Configurations}

The cations $M$ (alkali, alkaline earth, lanthanide) surrounding the complexes have various configurations, of which the eightfold cubic (or approximately cubic) is by far the most common. Depending on the metal ratios $\mathrm{M} / \mathrm{T}$ the cubes are joined via corners, edges, or faces. Edge sharing occurs in $\mathrm{Sr}_{2} \mathrm{RuH}_{6}$ (II), $\mathrm{Sr}_{2} \mathrm{IrH}_{5}$ (III), $\mathrm{Na}_{2} \mathrm{PtH}_{4}$ (IV), $\mathrm{Mg}_{2} \mathrm{NiH}_{4}(\mathrm{~V}), \mathrm{K}_{2} \mathrm{PtH}_{4}$ (VI), and $\mathrm{K}_{3} \mathrm{ReH}_{6}$ (XL) that adopt one arrangement, and $\mathrm{Ca}_{4} \mathrm{Mg}_{4} \mathrm{Fe}_{3} \mathrm{H}_{22}$ (XX) and $\mathrm{Ca}_{4} \mathrm{Mg}_{4} \mathrm{Co}_{3} \mathrm{H}_{19}$ (XXXII) that adopt another. Corner sharing occurs in $\mathrm{Li}_{4} \mathrm{RuH}_{6}(\mathrm{XI})$. Corner and edge sharing occurs in $\mathrm{Mg}_{3} \mathrm{ReH}_{7}$ (XXIII), $\mathrm{Mg}_{6} \mathrm{Co}_{2} \mathrm{H}_{11}$ (XVII), $\mathrm{Mg}_{3} \mathrm{RuH}_{6}$ (XXV), $\mathrm{LiMg}_{2} \mathrm{RuH}_{7}$ (XXVII), and $\mathrm{Mg}_{6} \mathrm{Ir}_{2} \mathrm{H}_{11}$ (XLV) that adopt one arrangement, and $\mathrm{Sr}_{2} \mathrm{MgFeH}_{8}$ (XVI), $\mathrm{LiMg}_{4} \mathrm{Os}_{2} \mathrm{H}_{13}$ (XXXVI), and $\mathrm{LiMg}_{4} \mathrm{Os}_{2} \mathrm{H}_{13}$ (XXXVI) that adopt another. Sheets of face-sharing cubes occur in $\mathrm{Mg}_{2} \mathrm{RuH}_{4}$ (XVIII) and $\mathrm{Ba}_{3} \mathrm{Ir}_{2} \mathrm{H}_{12}$ (XXXI) for which the sheets are connected via edges, and in $\mathrm{LiSr}_{2} \mathrm{PdH}_{5}$ (XXII) for which the sheets are connected via additional $\mathrm{M}$ atoms, and in $\mathrm{Na}_{2} \mathrm{PdH}_{2}$ (VII), $\mathrm{Ba}_{2} \mathrm{PtH}_{6}$ (XXI), and $\mathrm{Li}_{2} \mathrm{PtH}_{2}$ (XXXVII) that have other arrangements. Three-dimensional arrays of face-sharing cubes occur in $\mathrm{CaPdH}_{2}(\mathrm{X})$ and $\mathrm{MgRhH}_{1-x}$ (XIV) where all faces are shared, in $\mathrm{Sr}_{8} \mathrm{Rh}_{5} \mathrm{H}_{23}$ (XXVI) where edges, edges and faces, or faces are shared, and in $\mathrm{Ca}_{8} \mathrm{Rh}_{6} \mathrm{H}_{24}$ (XLI) where edges and faces are 
shared. Face-sharing configurations are of particular interest because they favor interactions between neighboring hydride complexes.

The distortion of the $M$ cube depends mainly on the symmetry and dynamics of the hydrido complex. This can be seen in $\mathrm{Mg}_{2} \mathrm{CoH}_{5}$ (III) and $\mathrm{Mg}_{2} \mathrm{NiH}_{4}(\mathrm{~V})$, whose T-metal centered $\mathrm{Mg}$ cubes are regular in the disordered cubic HT structure (such as in $\mathrm{Mg}_{2} \mathrm{FeH}_{6}$; II), and strongly distorted in the tetragonal $\left(\mathrm{Mg}_{2} \mathrm{CoH}_{5}\right)$ and monoclinic $\left(\mathrm{Mg}_{2} \mathrm{NiH}_{4}\right) \mathrm{LT}$ structures owing to ordering of the spy- $\mathrm{CoH}_{5}$ and tet- $\mathrm{NiH}_{4}$ units, respectively. Another example is $\mathrm{Mg}_{2} \mathrm{RuH}_{4}$ (XVIII) whose $\mathrm{Mg}$ cubes surrounding the sad- $\mathrm{RuH}_{4}$ units are strongly distorted compared to the regular $\mathrm{Mg}$ cubes surrounding the oct- $\mathrm{RuH}_{6}$ units in $\mathrm{Mg}_{2} \mathrm{RuH}_{6}$ (II). The influence of the complex geometry on the metal atom array is also apparent in $\mathrm{Mg}_{6} \mathrm{Co}_{2} \mathrm{H}_{11}$ (XVII), $\mathrm{Mg}_{6} \mathrm{Ir}_{2} \mathrm{H}_{11}$ (XLV), and $\mathrm{Mg}_{3} \mathrm{RuH}_{6}$ (XXV). They contain various nonoctahedral $\mathrm{TH}_{4}$ and $\mathrm{TH}_{5}$ units, and their metal substructure derives from that of trigonal $\mathrm{Mg}_{3} \mathrm{ReH}_{7}$ (XXIII) containing octahedral $\mathrm{ReH}_{6}$ units by an orthorhombic (or monoclinic) distortion. Finally, the atomic size difference between alkaline earths also plays a role, as can be seen from the severe distortions of the partially substituted $\mathrm{M}$ cubes in the quaternary hydrides $\mathrm{Ca}_{4} \mathrm{Mg}_{4} \mathrm{Fe}_{3} \mathrm{H}_{22}$ (XX) and $\mathrm{CaMgNiH}_{4}$ (XIX) that derive from $\mathrm{Mg}_{2} \mathrm{FeH}_{6}$ (II) and $\mathrm{Mg}_{2} \mathrm{NiH}_{4}$ (V), respectively.

\subsection{Bond Distances}

Metal-Hydrogen Bonds. The T-H bond distances of the complexes can be rationalized in terms of covalent radii and a fixed hydrogen radius of $0.28 \AA$. The distances range from $1.50-1.60 \AA$ for $3 \mathrm{~d}$ metals, to $1.70-1.80 \AA$ for $4 \mathrm{~d}$ and $5 \mathrm{~d}$ metals, except for Pd (1.60-1.70 $\AA$ ) and Pt (1.58-1.67 $\AA)$, the $\mathrm{T}-\mathrm{H}$ bonds of which are shortened owing to low-coordination numbers. Apparent bond shortening may also occur as a result of partial hydrogen site occupancy in disordered HT structures. On the other hand, the relatively long $\mathrm{Pd}-\mathrm{D}$ distances in disordered $\mathrm{CaPdD}_{2}(\mathrm{X})(\mathrm{Pd}-\mathrm{D}=1.84 \AA)$ and $\mathrm{LiSr}_{2} \mathrm{PdD}_{5}$ (XXII) $(\mathrm{Pd}-\mathrm{D}=1.95 \AA)$ are only averages, and are presumably shortened locally owing to the formation of linear $\mathrm{PdD}_{2}$ groups. Owing to matrix effects, ${ }^{\mathbf{1 2 7}}$ the $\mathrm{T}-\mathrm{D}$ bond lengths generally increase with the size of the M cations.

The $\mathrm{M}-\mathrm{H}$ distances are consistent with tabulated ionic radii. Those involving hydride anions $\mathrm{H}^{-}$surrounded by cations $\mathrm{M}$ only (occurring in some 25 compounds from 19 different structure types) are generally shorter than those involving hydrogen ligands of the complex. Exceptions are found in $\mathrm{K}_{3} \mathrm{PdH}_{3}$ (IX), $\mathrm{K}_{3} \mathrm{ZnH}_{5}$ and $\mathrm{K}_{3} \mathrm{MnH}_{5}$ (XXX), $\mathrm{Ba}_{7} \mathrm{Cu}_{3} \mathrm{H}_{17}$ (XXXV), $\mathrm{Rb}_{3} \mathrm{ReH}_{10}$ (XLII), and $\mathrm{Cs}_{3} \mathrm{OsH}_{9}$ (XLIV). Unlike the complex hydrogen ligands, the hydride anions are neither mobile nor disordered. Their metal configurations range from octahedral in $\mathrm{LiSr}_{2} \mathrm{PdH}_{5}$ (XXII), $\mathrm{K}_{3} \mathrm{PdD}_{3}$ (IX), $\mathrm{K}_{3} \mathrm{ZnH}_{5}$ (XXX), $\mathrm{Rb}_{3} \mathrm{ReH}_{10}$ (XLII),
$\mathrm{Cs}_{3} \mathrm{OsH}_{9}$ (XLIV), and $\mathrm{LaMg}_{2} \mathrm{NiH}_{7}$ (XLVII), to trigonal bipyramidal in $\mathrm{LiMg}_{2} \mathrm{RuH}_{7}$ (XXVII) and $\mathrm{LiMg}_{4} \mathrm{Os}_{2} \mathrm{H}_{13}$ (XXXVI), tetrahedral in $\mathrm{SrMg}_{2} \mathrm{FeH}_{8}$ (XVI), $\mathrm{Ca}_{4} \mathrm{Mg}_{4} \mathrm{Fe}_{3} \mathrm{H}_{22}$ (XX), $\mathrm{Ca}_{4} \mathrm{Mg}_{4} \mathrm{Co}_{3} \mathrm{H}_{19}$ (XXXII), $\mathrm{Ba}_{7} \mathrm{Cu}_{3} \mathrm{H}_{17}$ (XXXV), and $\mathrm{BaMg}_{2} \mathrm{RuH}_{8}$ (XXXVIII), triangular in $\mathrm{Mg}_{6} \mathrm{Co}_{2} \mathrm{H}_{11}$ (XVII) and $\mathrm{LaMg}_{2} \mathrm{NiH}_{7}$ (XLVII), and linear in $\mathrm{Mg}_{3} \mathrm{ReH}_{7}$ (XXIII) and $\mathrm{Mg}_{3} \mathrm{RuH}_{6}$ (XXV). As expected, low-coordinate $\mathrm{M}-\mathrm{H}$ bonds are significantly shorter, for example, $\mathrm{Mg}-\mathrm{H}=1.74 \AA$ in $\mathrm{Mg}_{6} \mathrm{Ir}_{2} \mathrm{H}_{11}$ (XLV) and $\mathrm{K}-\mathrm{H}=2.73 \AA$ in $\mathrm{K}_{3} \mathrm{PdH}_{5}$ (VIII) compared to higher coordinate $\mathrm{M}-\mathrm{H}$ bonds such as in binary $\mathrm{MgH}_{2}(1.95 \AA), \mathrm{BaH}_{2}(2.57 \AA)$ and $\mathrm{KH}(2.85 \AA)$. As in saline ternary hydrides, the $\mathrm{Yb}-\mathrm{D}$ distances are close to $\mathrm{Ca}-\mathrm{D}$ distances and the $\mathrm{Eu}-\mathrm{D}$ distances close to $\mathrm{Sr}-\mathrm{D}$ distances. $^{\mathbf{1 2 8}}$

Metal-Metal Bonds. The T- and M-metals are likely to interact in complex hydride structures because of their proximity. The shortest $\mathrm{M}-\mathrm{T}$ distances are in the range $2.5-3.5 \AA$ and give rise to metallic properties in metal-rich compounds, such as $\mathrm{MgRhD}_{1-x}(\mathrm{XIV})(\mathrm{Mg}-\mathrm{Rh}=2.66 \AA)$ and $\mathrm{Na}_{2} \mathrm{PdH}_{2}$ (VII) $(\mathrm{Na}-\mathrm{Pd}=3.13 \AA$ ). The shortest $\mathrm{M}-\mathrm{M}$ distances are in the range $2.5-4.5 \AA$ and come close to the sum of covalent radii, such as $\mathrm{Mg}-\mathrm{Mg}=2.75 \AA$ in $\mathrm{Mg}_{4} \mathrm{IrH}_{5}$ (XXIV), $2.78 \AA$ in $\mathrm{Mg}_{6} \mathrm{Co}_{2} \mathrm{H}_{11}$ (XVII) or $2.79 \AA$ in $\mathrm{Mg}_{6} \mathrm{Ir}_{2} \mathrm{H}_{11}$ (XLV). Clearly, these $\mathrm{M}-\mathrm{T}$ and $\mathrm{M}-\mathrm{M}$ interactions are not taken into account by a limiting ionic bond description and require more sophisticated electron counting schemes (see 4.4). Interactions between T-atoms are less common, because the $\mathrm{T}-\mathrm{T}$ distances usually exceed $3.5 \AA$. However, $\mathrm{T}-\mathrm{T}$ interactions do occur in metallic $\mathrm{Li}_{2} \mathrm{PdH}_{2}$ (VII) (Pd-Pd = 3.11 $\mathrm{A}$ ) and $\mathrm{Na}_{2} \mathrm{PdH}_{2}(\mathrm{VII})$ $(\mathrm{Pd}-\mathrm{Pd}=3.60 \AA)$, and possibly also in $\mathrm{Mg}_{2} \mathrm{RuH}_{4}$ (XVIII) $(\mathrm{Ru}-\mathrm{Ru}=3.24 \AA)$ and $\mathrm{Mg}_{3} \mathrm{RuH}_{3}(\mathrm{XV})(\mathrm{Ru}-\mathrm{Ru}=3.31 \AA)$ as suggested by their stereochemistry and the existence of carbonyls displaying such interactions across similar long distances.

Hydrogen-Hydrogen Contacts. The $\mathrm{H}-\mathrm{H}$ contacts in ordered hydride structures usually exceed $2.1 \AA$, and thus indicate nonbonding (or repulsive) $\mathrm{H}-\mathrm{H}$ interactions. In disordered structures, $\mathrm{H}$ sites closer than $2.1 \AA$ are always half (or less) occupied. The shortest $\mathrm{H}-\mathrm{H}$ contact occurs in $\mathrm{K}_{2} \mathrm{ReD}_{9}$ (I) for which the most recently reported value is $\mathrm{D}-\mathrm{D}=1.90 \AA \AA^{24}$ Thus, there is no evidence for hydrogen pairing in homoleptic solid-state T-metal hydrides, unlike in molecular T-metal hydride complexes for which dihydrogen groups with $\mathrm{H}-\mathrm{H}$ distances as short as $0.82 \AA$ do occur. ${ }^{10,129}$

\subsection{Bonding}

The compositions and ligand geometries of most mononuclear T-metal hydride complexes can be rationalized in terms of conventional electron counts based on full charge 
transfer from the surrounding cation matrix $\mathrm{M}$, and s-p$\mathrm{d}$ hybridization schemes that involve two-center-two-electron $(2 \mathrm{c}-2 \mathrm{e})$ bonds, such as $\left(\mathrm{d}^{0}\right) \mathrm{d}^{5} \mathrm{sp}^{3}$ for 18 -electron ttp- $\left[\mathrm{ReH}_{9}\right]^{2-}$, $\left(\mathrm{d}^{6}\right) \mathrm{d}^{2} \mathrm{sp}^{3}$ for octahedral 18-electron $\left[\mathrm{FeH}_{6}\right]^{4-},\left[\mathrm{MnH}_{6}\right]^{5-}$, and $\left[\mathrm{ReH}_{6}\right]^{5-},\left(\mathrm{d}^{8}\right) \mathrm{dsp}^{3}$ for square-pyramidal 18-electron $\left[\mathrm{CoH}_{5}\right]^{4-},\left(\mathrm{d}^{10}\right) \mathrm{sp}^{3}$ for 18-electron tetrahedral $\left[\mathrm{NiH}_{4}\right]^{4-}$ and $\left[\mathrm{PdH}_{4}\right]^{4-},\left(\mathrm{d}^{8}\right) \mathrm{dsp}^{2}$ for planar 16-electron $\left[\mathrm{PdH}_{4}\right]^{2-}$ and $\left[\mathrm{RhH}_{4}\right]^{3-},\left(\mathrm{d}^{10}\right) \mathrm{sp}^{2}$ for triangular 16-electron $\left[\mathrm{PdH}_{3}\right]^{3-}$, and $\left(\mathrm{d}^{10}\right) \mathrm{sp}$ for linear 14-electron $\left[\mathrm{PdH}_{2}\right]^{2-}$. However, for some hydrides, in particular those containing early T-metals and/or showing metal-metal interactions, more elaborate electron counting schemes evoking s-d hybridization only, three-center-four-electron $(3 \mathrm{c}-4 \mathrm{e})$ bonds, and partial charge transfer from the cation matrix $\mathrm{M}$ are also possible, as was shown by Firman and Landis. ${ }^{13} \mathrm{Mg}_{2} \mathrm{CoH}_{5}$ (III), for example, can be formulated in terms of univalent $\mathrm{Mg}^{+}$and $\mathrm{sd}^{2}$ hybridized 16-electron $\left[\begin{array}{lll}\mathrm{CoH}_{3} & 2 \mathrm{H}^{-}\end{array}\right]$ complexes having two $3 \mathrm{c}-4 \mathrm{e}$ bonds. The merits and limitations of this bonding model have been discussed by King. ${ }^{16}$ For polynuclear hydride complexes, the bonding situation is more difficult to describe. Some can be rationalized by mixtures between two-center-two-electron and three-center-two-electron $(3 \mathrm{c}-2 \mathrm{e})$ bonds, such as dinuclear $\left[\mathrm{H}_{4} \mathrm{Pt}-\mathrm{H}-\mathrm{PtH}_{4}\right]^{5-}$ in $\mathrm{Li}_{5} \mathrm{Pt}_{2} \mathrm{H}_{9}$ (XXXIV) for which eight $2 \mathrm{c}-2 \mathrm{e}$ bonds (terminal $\mathrm{H}$ 's) and one $3 \mathrm{c}-2 \mathrm{e}$ bond (bridging $\mathrm{H}$ ) can be formulated, while for others such as tetranuclear $\left[\mathrm{Ni}_{4} \mathrm{H}_{\sim 4}\right]^{5-}$ in $\mathrm{LaMgNi}_{4} \mathrm{H}_{\sim 4}$ (XLVI) a similar bond description is less apparent.

As expected from its small atomic size and high-field ligand character, hydrogen favors 18-electron complexes (about 40 representatives with groups 7, 8, 9, and 10 elements). Complexes with 16 electrons (some 11 representatives with $\mathrm{Re}, \mathrm{Ru}, \mathrm{Rh}, \mathrm{Pd}$, and $\mathrm{Pt}$ ), or 14 electrons (4 representatives with $\mathrm{Pd}$ and $\mathrm{Pt}$ ) are less numerous. The only octahedral 16 electron $\left(\mathrm{d}^{4}\right) \mathrm{d}^{2} \mathrm{sp}^{3}$ bonded complex is $\left[\mathrm{ReH}_{6}\right]^{3-}$ in $\mathrm{K}_{3} \mathrm{ReH}_{6}(\mathrm{XL})$, and the only tetrahedral 13-electron $\left(\mathrm{d}^{5}\right) \mathrm{sp}^{3}$ bonded complex is $\left[\mathrm{MnH}_{4}\right]^{2-}$ in $\mathrm{K}_{3} \mathrm{MnH}_{5}(\mathrm{XXX})$ that displays a half-filled $\mathrm{d}$ shell. On the other hand, the relative softness of hydrogen as a ligand and its stability as an anion favors structural diversity. This is illustrated by 'composite' hydrides such as quaternary $\mathrm{Ca}_{4} \mathrm{Mg}_{4} \mathrm{Fe}_{3} \mathrm{H}_{22}$ (XX) that derives from ternary $\mathrm{Mg}_{2} \mathrm{FeH}_{6}$ (II) by partial substitution of a fourfold negative $\left[\mathrm{FeH}_{6}\right]^{4-}$ by four $\mathrm{H}^{-}$, and $\mathrm{Mg}_{3} \mathrm{ReH}_{7}$ (XXIII), $\mathrm{Mg}_{6} \mathrm{Co}_{2} \mathrm{H}_{11}$ (XVII), and $\mathrm{Mg}_{3} \mathrm{RuH}_{6}$ (XXV) that have a common metal substructure and are capable of accommodating various complex geometries and $\mathrm{H}^{-}$ions.

The formal oxidation numbers of the T-metal in most hydride structures range between $-\mathrm{I}(\mathrm{Co}, \mathrm{Rh})$, zero $(\mathrm{Ru}$, $\mathrm{Ni}, \mathrm{Pd}$ ), I (Mn, Re, Co, Ir, Rh, Cu), II (Fe, Ru, Os, Pd, $\mathrm{Pt}, \mathrm{Zn}$ ), III (Ir, Rh), IV (Ru, Os, Pt), VI (Os) and VII (Re). They are consistent with configurational differences as found, for example, in palladates that change from tetrahedral as in $\left[\mathrm{Pd}^{0} \mathrm{H}_{4}\right]^{4-}$ to planar as in $\left[\mathrm{Pd}^{\mathrm{II}} \mathrm{H}_{4}\right]^{2-}$. As expected, high formal oxidation numbers occur mainly with heavier $\mathrm{T}$ elements such as $\mathrm{Re}^{\mathrm{VII}}$ in $\left[\mathrm{ReH}_{9}\right]^{2-}, \mathrm{Os}^{\mathrm{VI}}$ in $\left[\mathrm{OsH}_{8}\right]^{2-}$, and $\mathrm{Pt}^{\mathrm{IV}}$ in $\left[\mathrm{PtH}_{6}\right]^{2-}$, for which no $3 \mathrm{~d}$ analogs exist. As the $\mathrm{H} / \mathrm{M}$ ratios decrease, the systems tend to become metallic and electron counting becomes less obvious. Illustrative examples are $\mathrm{Mg}_{3} \mathrm{RuH}_{3}$ (XV) and $\mathrm{Mg}_{4} \mathrm{IrH}_{5}$ (XXIV) for which the neglect of $\mathrm{Mg}-\mathrm{Mg}$ bonding leads to unreasonable electron configurations and formal oxidation numbers (-III for $\mathrm{Ru}$ and Ir). Clearly, limiting ionic formulas for such hydrides are misleading, at least from a bonding point of view, but they are still useful for rationalizing hydrogen contents, which is generally not possible with 'interstitial' (metallic) metal hydrides.

Finally, an important bonding feature of complex metal hydrides are the interactions between the complex hydrogen ligands and the surrounding cation matrix. As can be seen from the isoelectronic series $\mathrm{Mg}_{2} \mathrm{FeH}_{6}-\mathrm{Mg}_{2} \mathrm{CoH}_{5}-\mathrm{Mg}_{2} \mathrm{NiH}_{4}$, the cations adopt cubic - or nearly cubic-configurations that maximize the $\mathrm{Mg}-\mathrm{H}$ interactions. Clearly such $\mathrm{M}-\mathrm{H}$ interactions not only help stabilizing the complexes but also contribute to the thermal stability of the overall structure, as shown by the desorption enthalpies of substitution pairs such as $\mathrm{Mg}_{2} \mathrm{NiH}_{4}-\mathrm{CaMgNiH}_{4}$ and $\mathrm{Mg}_{2} \mathrm{FeH}_{6}-\mathrm{Ca}_{4} \mathrm{Mg}_{4} \mathrm{Fe}_{3} \mathrm{H}_{22}$ (see Section 5). On the other hand, $\mathrm{T}-\mathrm{H}$ interactions do not much contribute to thermal stability as suggested by the relatively weak force constants as measured in metal hydrides such as $\mathrm{Mg}_{2} \mathrm{FeH}_{6}(\mathrm{Fe}-\mathrm{H}$ stretching mode $\sim 1.9$ mdyne $\left.\AA^{-1} ; \mathrm{Fe}-\mathrm{H}=1.56 \AA\right),{ }^{38}$ and the significant elongation of the $\mathrm{T}-\mathrm{H}$ bonds in the thermally more stable analog $\mathrm{Ca}_{2} \mathrm{FeH}_{6}(\mathrm{Fe}-\mathrm{H}=1.62 \AA)$. Theoretical band structure calculations are available for some of these systems, for example, $\mathrm{Mg}_{3} \mathrm{MnH}_{7}$ (XXIII), ${ }^{90} \mathrm{Na}_{2} \mathrm{PdH}_{4}$ (IV), $\mathrm{Na}_{2} \mathrm{PdH}_{2}$ (VII), $\mathrm{Li}_{2} \mathrm{PdH}_{2}$ (VII), $\mathrm{NaBaPdH}_{3}$ (XXXIX), and $\mathrm{Ba}_{2} \mathrm{PdH}_{4}$ (XXIX), ${ }^{14} \mathrm{Mg}_{2} \mathrm{NiH}_{4}$ (V), $\mathrm{Mg}_{2} \mathrm{CoH}_{5}$ (III), $\mathrm{Mg}_{2} \mathrm{TH}_{6}$ (II; $\mathrm{T}=\mathrm{Fe}, \mathrm{Ru}, \mathrm{Os}$ ), $\mathrm{M}_{2} \mathrm{FeH}_{6}$ (II; $\mathrm{M}=\mathrm{Mg}, \mathrm{Ca}, \mathrm{Sr}$ ), ${ }^{37} \mathrm{APdH}_{3}$ $(\mathrm{X} ; \mathrm{A}=\mathrm{Sr}, \mathrm{Eu}, \mathrm{Yb}){ }^{71} \mathrm{BaReH}_{9}(\mathrm{XXVIII}),{ }^{98}$ and $\mathrm{Eu}_{2} \mathrm{PdH}_{4}$ (XXIX), ${ }^{103}$ but have not yet addressed the issue of thermal stability.

\section{PROPERTIES}

In contrast to their 'interstitial' counterparts, complex metal hydrides have no apparent homogeneity range and are usually nonmetallic. Many are colored (greenish: $\mathrm{Mg}_{2} \mathrm{FeH}_{6}, \quad \mathrm{Na}_{4} \mathrm{RuH}_{6}, \quad \mathrm{SrMg}_{2} \mathrm{FeH}_{8}, \quad \mathrm{LiSr}_{2} \mathrm{PdH}_{5} ;$ reddish: $\mathrm{Eu}_{2} \mathrm{RuH}_{6}, \mathrm{Mg}_{2} \mathrm{RuH}_{4}$; red-violet: $\mathrm{Na}_{2} \mathrm{PtH}_{4}$; yellow: $\mathrm{K}_{3} \mathrm{PdH}_{3}$; yellow-green: $\mathrm{K}_{2} \mathrm{PdH}_{4}$; brownish: $\mathrm{Mg}_{2} \mathrm{RuH}_{6}, \mathrm{Mg}_{2} \mathrm{NiH}_{4}$, $\mathrm{Mg}_{3} \mathrm{RuH}_{6}, \mathrm{Ca}_{4} \mathrm{Mg}_{4} \mathrm{Fe}_{3} \mathrm{H}_{22}, \mathrm{BaMg}_{2} \mathrm{FeH}_{8}$ ), while some are colorless $\left(\mathrm{K}_{2} \mathrm{PtH}_{4}, \mathrm{~K}_{3} \mathrm{PtH}_{5}, \mathrm{Na}_{3} \mathrm{RhH}_{6}\right)$, or appear as white $\left(\mathrm{Mg}_{2} \mathrm{OsH}_{6}, \mathrm{Li}_{4} \mathrm{RuH}_{6}\right)$ or gray powders $\left(\mathrm{Ca}_{2} \mathrm{RuH}_{6}, \mathrm{Mg}_{3} \mathrm{RuH}_{3}\right.$, $\left.\mathrm{Mg}_{3} \mathrm{ReH}_{7}\right)$. Only few are black $\left(\mathrm{Yb}_{2} \mathrm{RuH}_{6}, \mathrm{Mg}_{6} \mathrm{Co}_{2} \mathrm{H}_{11}\right.$, $\left.\mathrm{Mg}_{2} \mathrm{CoH}_{5}, \mathrm{YbMgNiH}_{4}, \mathrm{Yb}_{4} \mathrm{Mg}_{4} \mathrm{Fe}_{3} \mathrm{H}_{22}\right)$ or have metallic luster $\left(\mathrm{Li}_{3} \mathrm{RhH}_{4}, \mathrm{Na}_{2} \mathrm{PdH}_{2}\right)$. Relatively well-characterized complex hydrides having metallic properties are $\mathrm{Li}_{2} \mathrm{PdH}_{2}$ and $\mathrm{Na}_{2} \mathrm{PdH}_{2}$ that are considered to be two-dimensional metals. 
Among complex hydrides that derive from intermetallic compounds, some show hydrogenation induced metal to nonmetal transitions such as brownish-red $\mathrm{Mg}_{2} \mathrm{NiH}_{4}$ $\left(\mathrm{Mg}_{2} \mathrm{Ni}\right)$, red $\mathrm{Mg}_{6} \mathrm{Ir}_{2} \mathrm{H}_{11}\left(\mathrm{Mg}_{3} \mathrm{Ir}\right)$, and dark grey $\mathrm{LaMg}_{2} \mathrm{NiH}_{7}$ $\left(\mathrm{LaMg}_{2} \mathrm{Ni}\right)$. Such transitions are of both fundamental and technological interest as shown for binary systems like $\mathrm{Y}-\mathrm{H}_{2}$ (switchable mirrors) and ternary systems like $\mathrm{Mg}_{2} \mathrm{Ni}-\mathrm{H}_{2}$ (tuneable optic windows). ${ }^{\mathbf{1 3 0}}$

Many hydrides are diamagnetic, as expected for closedelectron shells associated with low-spin $\mathrm{d}^{6}$ (oct), $\mathrm{d}^{8}$ ( $\mathrm{spl}$ ), and $\mathrm{d}^{10}$ (tet, lin) configurations. Others show weak temperature independent paramagnetism such as the $\mathrm{d}^{4}$ systems $\mathrm{K}_{3} \mathrm{ReH}_{6}$ (XL) and $\mathrm{Na}_{3} \mathrm{OsH}_{7}$ (XLIII). Finally, systems containing magnetic ions tend to order magnetically at low temperature, such as rose colored $\mathrm{K}_{3} \mathrm{MnH}_{5}\left(\mathrm{Mn}(\mathrm{II}), \mu_{\text {eff }}=4.5 \mu_{\mathrm{B}}, T_{\mathrm{N}}=\right.$ $28 \mathrm{~K})$ and faint violet colored $\mathrm{Eu}_{2} \mathrm{PdH}_{4}\left(\mathrm{Eu}(\mathrm{II}), \mu_{\mathrm{eff}}=\right.$ $\left.8 \mu_{\mathrm{B}}, T_{\mathrm{C}}=15 \mathrm{~K}\right)$.

Vibrational spectra as measured by INS on hydrides such as $\mathrm{M}_{2} \mathrm{TH}_{6}$ (II, $\mathrm{M}=\mathrm{Mg}, \mathrm{Ca}, \mathrm{Sr}, \mathrm{Ba} ; \mathrm{T}=\mathrm{Fe}, \mathrm{Ru}$, Os), ${ }^{28,38} \mathrm{Mg}_{2} \mathrm{NiH}_{4}(\mathrm{~V})$ and $\mathrm{Rb}_{3} \mathrm{ZnH}_{5}(\mathrm{XXX})^{\mathbf{5 4}}$ indicate $\mathrm{T}-\mathrm{H}$ stretching and bending modes in the expected ranges of $1600-2000 \mathrm{~cm}^{-1}$ and $800-1000 \mathrm{~cm}^{-1}$, respectively. These modes correlate with bonding properties, such as the $\mathrm{T}-\mathrm{H}$ stretching frequencies in the $\mathrm{K}_{2} \mathrm{PtCl}_{6}$ type series (II) that increase in the sequence $\mathrm{T}=\mathrm{Fe}, \mathrm{Ru}, \mathrm{Os}$, and decrease as the $\mathrm{M}-\mathrm{H}$ bond lengths increase. Their theoretical band gaps show the opposite trend, that is, they increase in the sequence $\mathrm{Mg}_{2} \mathrm{TH}_{6}(\mathrm{~T}=\mathrm{Fe}, \mathrm{Ru}$, Os) owing to the increasing atomic number of $\mathrm{T}$, and that decrease in the sequence $\mathrm{M}_{2} \mathrm{FeH}_{6}$ $(\mathrm{M}=\mathrm{Mg}, \mathrm{Ca}, \mathrm{Sr})$ owing to the increased participation of $\mathrm{M}$ d orbitals.

Properties of relevance for hydrogen storage applications, such as desorption enthalpies and weight and volume efficiencies, are summarized in Table 21.

Clearly, some compounds have outstanding properties, such as $\mathrm{Mg}_{2} \mathrm{FeH}_{6}$, which shows the highest known volume efficiency for hydrogen storage of all materials known (120 $\mathrm{g} \mathrm{H}_{2} \mathrm{~L}^{-1}$, that is, more than twice that of liquid

Table 21 Desorption enthalpies and hydrogen storage efficiencies

\begin{tabular}{lccc}
\hline & $\begin{array}{c}\Delta H \\
\mathrm{~kJ} \mathrm{~mol}^{-1} \mathrm{H}_{2}\end{array}$ & $\begin{array}{c}\text { Weight } \\
\text { efficiency } \\
\mathrm{wt} \%\end{array}$ & $\begin{array}{c}\text { Volume efficiency } \\
\mathrm{gH}_{2} \mathrm{~L}^{-1}\end{array}$ \\
\hline Complex hydrides & & & \\
$\mathrm{Mg}_{2} \mathrm{NiH}_{4}$ & 64 & 3.6 & 18 \\
$\mathrm{Mg}_{2} \mathrm{CoH}_{5}$ & 86 & 4.5 & 126 \\
$\mathrm{Mg}_{2} \mathrm{FeH}_{6}$ & 98 & 5.5 & 150 \\
$\mathrm{CaMgNiH}_{4}$ & 129 & 3.2 & 87 \\
$\mathrm{Ca}_{4} \mathrm{Mg}_{4} \mathrm{Fe}_{3} \mathrm{H}_{22}$ & 122 & 5.0 & 121 \\
$\mathrm{Binary} \mathrm{hydrides}_{\mathrm{MgH}_{2}}$ & 74 & 7.7 & 109 \\
$\mathrm{CaH}_{2}$ & $184^{a}$ & 4.8 & 92 \\
$\mathrm{Metallic} \mathrm{hydride}$ & 31 & 1.4 & 93 \\
$\mathrm{LaNi}_{5} \mathrm{H}_{6}$ & 31 & & \\
\hline
\end{tabular}

${ }^{\mathrm{a}}$ Model prediction. ${ }^{131}$ hydrogen), and $\mathrm{BaReH}_{9}$, which has an $\mathrm{H} / \mathrm{M}$ ratio that surpasses the hydrogen-to-carbon ratio of methane $(\mathrm{H} / \mathrm{C}=4)$. The weight efficiencies are also remarkable as shown by $\mathrm{Mg}_{2} \mathrm{FeH}_{6}$ and $\mathrm{Mg}_{3} \mathrm{MnH}_{7}$, which can store up to $5 \mathrm{wt} \%$ hydrogen, that is, more than currently used interstitial hydrides such as $\mathrm{AB}_{5} \mathrm{H}_{\sim 6}(\mathrm{~A}=\mathrm{La}$, etc., $\mathrm{B}=\mathrm{Ni}$, etc. $)$ and $\mathrm{AB}_{2} \mathrm{H}_{\sim 4}(\mathrm{~A}=\mathrm{Ti}$, etc., $\mathrm{B}=\mathrm{Mn}$, etc. $)$, which store only up to $\sim 2 \mathrm{wt} \%$ hydrogen. Unfortunately, regarding thermal stability complex metal hydrides perform less well than their interstitial counterparts. Only few hydrides decompose near room temperature such as $\mathrm{BaReH}_{9}$ and $\mathrm{Ba}_{7} \mathrm{Cu}_{3} \mathrm{H}_{17}$ that are, however, relatively heavy and expensive and not completely reversible. $\mathrm{Mg}_{2} \mathrm{NiH}_{4}$, which is the only commercialized hydride of this class, decomposes only above $523 \mathrm{~K}$, corresponding to a desorption enthalpy of $\Delta H=64 \mathrm{~kJ} \mathrm{~mol}^{-1}$ $\mathrm{H}_{2}$. Most other complex metal hydrides are more stable (decomposition temperatures $>573 \mathrm{~K}, \Delta H>80 \mathrm{~kJ} / \mathrm{H}_{2}$ ) and must be heated to yield hydrogen at useful pressures, which represents a penalty in energy. On the other hand, compounds such as $\mathrm{Mg}_{2} \mathrm{FeH}_{6}$ are of interest for high-temperature applications ${ }^{39}$ because of their high thermal stability and ease of cycling.

Owing to the scarcity of thermodynamic data, empirical models such as that used to rationalize thermal stabilities of interstitial metal hydrides ${ }^{\mathbf{1 3 1}}$ do not exist for complex metal hydrides. Metal-hydrogen interactions obviously play a role, as can be seen from the substitution pairs $\mathrm{Mg}_{2} \mathrm{NiH}_{4}-\mathrm{CaMgNiH}_{4}$ and $\mathrm{Mg}_{2} \mathrm{FeH}_{6}-\mathrm{Ca}_{4} \mathrm{Mg}_{4} \mathrm{Fe}_{3} \mathrm{H}_{22}$, whose stabilities increase strongly as one goes from the ternary $\mathrm{Mg}$ compounds to the quaternary $\mathrm{Ca}$ compounds (see Table 21). This trend correlates with the relatively strong interactions between hydrogen and calcium that forms a very stable binary hydride, compared to the relatively weak interactions of hydrogen with magnesium that forms a less stable binary hydride. This suggests that interactions between hydrogen and metal cations govern to a large extent the thermal stability of complex metal hydrides.

\section{CONCLUSIONS}

Solid-state transition metal hydride complexes occur mainly with the late transition elements. The complexes are usually mononuclear (centred by one T-metal atom only) and contain between two and nine terminal hydrogen ligands, while some are polynuclear (centred by more than one T-metal atom) and display bridging hydrogen ligands. The ligands tend to be disordered at higher temperatures and ordered at lower temperatures. The structural and electronic configurations of the hydrido complexes are consistent with those usually found in coordination compounds. They are stabilized by charge transfer from the surrounding cation matrix, are often 18electron, and less often 16- or 14-electron. Some hydride structures show evidence for metal-metal interactions, as 
in typically interstitial metal hydrides, while others contain additional hydride anions $\left(\mathrm{H}^{-}\right)$bonded to electropositive metals only, such as in saline hydrides. In metal-rich systems, hydrogen has interstitial character, as in typically metallic transition metal hydrides. No $\mathrm{H}-\mathrm{H}$ bond formation is observed. Most complex hydride systems are nonmetallic and many are colored. Some systems, however, show hydrogenation induced complex formation and metal-to-insulator transitions. They generally display very high volume efficiencies for hydrogen storage but are thermally too stable and/or too expensive for practical applications. Thermodynamic data suggest that their enthalpy of formation scales with the thermal stability of the binary hydrides of the electropositive metal constituents.

\section{RELATED ARTICLES}

Hydride Complexes of the Transition Metals.

\section{REFERENCES}

1. K. Yvon, Hydrides: Solid State Transition Metal Complexes, in 'Encyclopedia Inorganic Chemistry', ed. R. B. King, John Wiley, New York 1994, Vol. 3, p. 1401.

2. S. C. Abrahams, A. P. Ginsberg, and K. Knox, Inorg. Chem., 1964, 3, 558; see also K. Knox and A. P. Ginsberg, Inorg. Chem., 1964, 3, 555; for $\mathrm{K}_{2} \mathrm{TcH}_{9}$ see also A. P. Ginsberg, Inorg. Chem., 1964, 3, 567.

3. R. O. Moyer Jr, C. Stanitski, fnmJ. Tanaka, M. I. Kay, and R. Kleinberg, J. Solid State Chem., 1971, 3, 541; for a review of work by these authors see R. O. Moyer Jr, R. Linsay, and D. N. Marks, Adv. Chem. Ser., 1978, 167, 366.

4. L. Schlapbach, Top. Appl. Phys., 1988, 63, 1; 1992, 67, 1; see also MRS Bull., 2002, 27/9, 675.

5. J. J. Reilly and R. H. Wiswall, Inorg. Chem., 1968, 7, 2254.

6. P. Zolliker, K. Yvon, J. D. Jorgensen, and F. J. Rotella, Inorg. Chem., 1986, 25, 3590; see also K. Yvon, J. Schefer, and F. Stucki, Inorg. Chem., 1981, 20, 2776.

7. W. Bronger, Angew. Chem., Int. Ed. Engl., 1991, 30, 759.

8. W. Bronger, J. Alloys Comp., 1995, 229, 1.

9. W. Bronger and G. Auffermann, Chem. Mater., 1998, 10, 2723.

10. R. Bau and M. H. Drabnis, Inorg. Chim. Acta, 1997, 259, 27.

11. K. Yvon, Z. Kristallogr., 2003, 218, 108.

12. G. J. Miller, H. Deng, and R. Hoffmann, Inorg. Chem., 1994, 33, 1330 .
13. T. K. Firman and C. R. Landis, J. Am. Chem. Soc., 1998, 120, 12650.

14. M. Olofsson-Martensson, U. Häussermann, J. Tomkinson, and D. Noréus, J. Am. Chem. Soc., 2000, 122, 6960.

15. K. Yvon, Chimia, 1998, 52, 613.

16. R. B. King, Coord. Chem. Rev., 2000, 200-202, 813.

17. K. Yvon, Swiss Neutron News, 2002, 22, 11.

18. K. Yvon, 'Encyclopedia of Materials: Science and Technology Updates', ed. K. H. J. Buschow, Elsevier Ltd., 2004, pp. 1-9.

19. W. Grochala and P. P. Edwards, Chem. Rev., 2004, 104, 1283.

20. G. Sandrock, and G. Thomas, 1997, Hydrogen Information Center, http://hydpark.ca.sandia.gov/, IEA/DOE/SNL Hydride Databases.

21. R. Bau, D. M. Ho, and S. G. Gibbins, J. Am. Chem. Soc., 1981, 103, 4960; see also D. E. Linn Jr, G. M. Skidd, and E. M. Tippmann, Inorg. Chim. Acta, 1999, 291, 142.

22. R. G. Teller and R. Bau, Struct. Bonding (Berlin), 1981, 44, 1; see also R. Bau, M. Y. Chiang, D. M. Ho, S. G. Gibbins, T. J. Emge, and T. F. Koetzle, Inorg. Chem., 1984, 23, 2823.

23. K. Yvon and P. Fischer, Top. Appl. Phys., 1988, 63, 87 (Springer).

24. W. Bronger, L. à Brassard, P. Müller, B. Lebech, and Th. Schultz, Z. Anorg. Allg. Chem., 1999, 625, 1143.

25. R. Lindsay, R. O. Moyer, J. S. Thompson, and D. Kuhn, Inorg. Chem., 1976, 15, 3050; see also R. Lindsay and R. O. Moyer Jr, Inorg. Chem., 1981, 80, P37; see also R. O. Moyer Jr, R. Lindsay, and D. F. Storey, Z. Phys. Chem. Neue Folge, 1989, 165, 83; see also R. Lindsay, R. O. Moyer, W. Strange, W. H. Klapp, D. F. Storey, and J. R. Knapp, Z. Phys. Chem. Neue Folge, 1993, 179, 457; for $\mathrm{Eu}_{2} \mathrm{IrH}_{5}$ see R. O. Moyer $\mathrm{Jr}$ and R. Lindsay, J. Less-Common Met., 1980, 70, P57.

26. J.-J. Didisheim, P. Zolliker, K. Yvon, P. Fischer, J. Schefer, M. Gubelmann, and A. F. Williams, Inorg. Chem., 1984, 23, 1953.

27. M. Kritikos, D. Noréus, B. Bogdanovic, and U. Wilczok, $J$. Less-Common Met., 1990, 161, 337.

28. M. Kritikos and D. Noréus, J. Solid State Chem., 1991, 93, 256.

29. B. Huang, F. Bonhomme, P. Selvam, K. Yvon, and P. Fischer, J. Less-Common Met., 1991, 171, 301.

30. P. Selvam and K. Yvon, Int. J. Hydrogen Energy, 1991, 16, 615.

31. J. Huot, H. Hayakawa, and E. Akiba, J. Alloys Comp., 1997, 248, 164; see also J. Huot, S. Boily, E. Akiba, and R. Schulz, J. Alloys Comp., 1998, 280, 306. 
32. A. Hightower, B. Fultz, and R. C. Bowman Jr, J. Alloys Comp., 1997, 252, 238; see also S. S. Sai Raman, D. J. Davidson, J.-L. Bobet, and O. N. Srivastava, J. Alloys Comp., 2002, 333, 282; see also F. C. Gennari, F. J. Castro, and J. J. A. Gamboa, J. Alloys Comp., 2002, 339, 261.

33. W. Bronger and G. Auffermann, Angew. Chem., Int. Ed. Engl., 1994, 33, 1112.

34. W. Bronger and G. Auffermann, J. Alloys Comp., 1995, 219, 45.

35. W. Bronger and G. Auffermann, Z. Anorg. Allg. Chem., 1995, 621, 1318.

36. J. S. Thompson, R. O. Moyer, and R. Lindsay, Inorg. Chem., 1975, 14, 1866; see also R. Lindsay, R. O. Moyer Jr, W. Strange, and B. J. Burnim, J. Alloys Comp., 1996, 243, 90; for $\mathrm{Yb}_{2} \mathrm{RuH}_{6}$ see R. Lindsay, R. O. Moyer Jr, J. S. Thompson, and D. Kuhn, Inorg. Chem., 1976, 15, 3050 .

37. E. Orgaz and M. Gupta, Z. Phys. Chem., 1993, 181, 1; see also M. Gupta and L. Schlapbach, Top. Appl. Phys., 1988, 63, 139; see also M. Gupta, J. less-Common Met., 1984, 103, 325.

38. S. F. Parker, K. P. J. Williams, M. Bortz, and K. Yvon, Inorg. Chem., 1997, 36, 5218.

39. B. Bogdanovic, A. Reiser, K. Schlichte, B. Spliethoff, and B. Tesche, J. Alloys Comp., 2002, 345, 77.

40. R. O. Moyer Jr, J. R. Wilkins, and P. Ryan, J. Alloys Comp., 1999, 290, 103.

41. J. Zhuang, J. M. Hastings, L. M. Corliss, R. Bau, C.-Y. Wei, and R. O. Moyer Jr, J. Solid State Chem., 1981, 40, 352.

42. J. Zhuang, W. Kunnmann, L. M. Corliss, J. M. Hastings, and R. O. Moyer Jr, J. Solid State Chem., 1983, 48, 117; for $\mathrm{Ca}_{2} \mathrm{IrD}_{5}$ see R. O. Moyer Jr and B. H. Toby, J. Alloys Comp., 2004, 363, 99.

43. H. Kohlmann, R. O. Moyer, T. Hansen, and K. Yvon, J. Solid State Chem., 2003, 174, 35.

44. P. Zolliker, K. Yvon, P. Fischer, and J. Schefer, Inorg. Chem., 1985, 24, 4177.

45. M. Yoshida, F. Bonhomme, K. Yvon, and P. Fischer, J. Alloys Comp., 1993, 190, L45.

46. W. Bronger, K. Jansen, and L. Breil, Z. Anorg. Allg. Chem., 1998, 624, 1477.

47. W. Bronger, R. Beissmann, and G. Ridder, J. Alloys Comp., 1994, 203, 91.

48. F. Bonhomme, P. Selvam, M. Yoshida, K. Yvon, and P. Fischer, J. Alloys Comp., 1992, 178, 167.

49. R. O. Moyer Jr, B. J. Burnim, and R. Lindsay, J. Solid State Chem., 1996, 121, 56.

50. W. Bronger, P. Müller, D. Schmitz, and H. Spittank, Z. Anorg. Allg. Chem., 1984, 516, 35.

51. K. Kadir, M. Kritikos, D. Noréus, and A. F. Andresen, J. LessCommon Met., 1991, 172-174, 36.
52. M. Gupta, Z. Phys. Chem., 1993, 181, 9.

53. W. Bronger and G. Auffermann, J. Alloys Comp., 1995, 228, 119.

54. S. F. Parker, K. P. J. Williams, T. Smith, M. Bortz, B. Bertheville, and K. Yvon, Phys. Chem. Chem. Phys., 2002, 4, 1732.

55. U. Häussermann, H. Blomqvist, and D. Noréus, Inorg. Chem., 2002, 41, 3684.

56. P. Zolliker, K. Yvon, and C. Baerlocher, J. Less-Common Met., 1986, 115, 65; see also D. Noréus and L. Kihlborg, J. Less-Common Met., 1986, 123, 233.

57. K. Yvon, J. Schefer, and F. Stucki, Inorg. Chem., 1981, 20, 2776.

58. E. Rönnebro, J. O. Jensen, D. Noréus, and N. J. Bjerrum, J. Alloys Comp., 1999, 293-295, 146.

59. W. Bronger, G. Auffermann, and P. Müller, J. Less-Common Met., 1986, 116, 9; see also W. Bronger, G. Auffermann, and P. Müller, J. Less-Common Met., 1988, 142, 243; see also W. Bronger, and G. Auffermann, J. Alloys Comp., 1992, 187, 87.

60. D. Noréus, K. W. Törnroos, A. Börje, T. Szabó, W. Bronger, H. Spittank, G. Auffermann, and P. Müller, J. Less-Common Met., 1988, 139, 233; see also K. Kadir and D. Noréus, Z. Phys. Chem. NF., 1989, 163, 231.

61. R. V. Kasowski, D. Noréus, L. Wang, and M.-H. Whangbo, Inorg. Chem., 1992, 31, 4737.

62. W. Bronger and G. Auffermann, J. Less-Common Met., 1991, 169, 173.

63. W. Bronger, G. A uffermann, and P. Müller, Z. Anorg. Allg. Chem., 1988, 566, 31; see also W. Bronger and G. Auffermann, J. Alloys Comp., 1992, 187, 81; W. Bronger and G. Auffermann, J. Alloys Comp., 1992, 179, 235.

64. W. Bronger and G. Auffermann, J. Less-Common Met., 1990, 158, 163.

65. W. Bronger, K. Jansen, and P. Müller, J. Less-Common Met., 1990, 161, 299.

66. B. Huang, Synthèse, Structure et Stabilité Thermique des Hydrures Ternaires et Quaternaires Complexes des Métaux de Transition, Thesis No 2765 (in English), University of Geneva, 1995.

67. W. Bronger and G. Ridder, J. Alloys Comp., 1994, 210, 53.

68. H. Kohlmann, H. E. Fischer, and K. Yvon, Inorg. Chem., 2001, 40, 2608; see also K. H. J. Buschow, R. L. Cohen, and K. W. West, J. Appl. Phys., 1977, 48, 5289.

69. K. Ensslen, E. Bucher, and H. Oesterreicher, J. Less-Common Met., 1983, 92, 343.

70. H. T. Takeshita, T. Oishi, and N. Kuriyama, J. Alloys Comp., 2002, 333, 266.

71. E. Orgaz, V. Mazel, and M. Gupta, J. Alloys Comp., 1997, 253-254, 330. 
72. M. Kritikos, D. Noréus, A. F. Andresen, and P. Fischer, J. Solid State Chem., 1991, 92, 514.

73. W. Bronger, M. Gehlen, and G. Auffermann, J. Alloys Comp., 1991, 176, 255.

74. W. Bronger, P. Müller, J. Kowalczyk, and G. Auffermann, J. Alloys Comp., 1991, 176, 263.

75. W. Bronger, M. Gehlen, and G. Auffermann, Z. Anorg. Allg. Chem., 1994, 620, 1983.

76. L. B. Lundberg, D. T. Cromer, and C. B. Magee, Inorg. Chem., 1972, 11, 400.

77. F. Bonhomme, K. Yvon, and P. Fischer, J. Alloys Comp., 1992, 186, 209; erratum, F. Bonhomme, K. Yvon, and P. Fischer, J. Alloys Comp., 1992, 190, 141; see also F. Bonhomme, Synthèse et Caractérisation Structurale d'Hydrures Ternaires Contenant du Magnésium et un Métal de Transition du Groupe VIII, Thesis No 2720 (in French), University of Geneva, 1995.

78. F. Bonhomme, K. Yvon, and P. Fischer, J. Alloys Comp., 1992, 186, 309.

79. B. Huang, K. Yvon, and P. Fischer, J. Alloys Comp., 1992, 187, 227; see also Huang, K. Yvon, and P. Fischer, J. Alloys Comp., 1995, 227, 121.

80. R. Černý, F. Bonhomme, K. Yvon, P. Fischer, P. Zolliker, D. E. Cox, and A. Hewat, J. Alloys Comp., 1992, 187, 233.

81. E. J. Ivanov, I. Konstanchuk, A. Stepanov, Yan. Jie, M. Pezat, and B. Darriet, Inorg. Chem., 1989, 28, 613; see also I. G. Konstanchuk, E. Yu. Ivanov, A. A. Stepanov, and T. I. Samsonova, Izv. Akad. Nauk SSSR, Ser. Khim. Nauk, 1989, 3, 93 (in Russian).

82. F. Bonhomme, K. Yvon, G. Triscone, K. Jansen, G. Auffermann, P. Müller, W. Bronger, and P. Fischer, J. Alloys Comp., 1992, 178, 161.

83. B. Huang, K. Yvon, and P. Fischer, J. Alloys Comp., 1992, 178, 173; see also B. Huang, K. Yvon, and P. Fischer, $J$. Alloys Comp., 1994, 204, L5.

84. F. Gingl and K. Yvon, Z. Kristallogr., 1993, 207, 247.

85. B. Huang, K. Yvon, and P. Fischer, J. Alloys Comp., 1992, 190, 65; see also B. Huang, K. Yvon, and P. Fischer, J. Alloys Comp., 1993, 197, 65.

86. K. Kadir and D. Noreus, Z. Phys. Chem., 1993, 179, 237.

87. M. Yoshida, K. Yvon, and P. Fischer, J. Alloys Comp., 1993, 194, L11.

88. B. Huang, K. Yvon, and P. Fischer, J. Alloys Comp., 1993, 197, 97.

89. M. Bortz, B. Bertheville, K. Yvon, E. A. Movlaev, V. N. Verbetzky, and F. Fauth, J. Alloys Comp., 1998, 279, L8.

90. E. Orgaz and M. Gupta, J. Alloys Comp., 1999, 330-332, 323.

91. F. Bonhomme, N. T. Stetson, K. Yvon, P. Fischer, and A. W. Hewat, J. Alloys Comp., 1993, 200, 65.
92. W. Bronger, K. Jansen, and G. Auffermann, J. Alloys Comp., 1993, 199, 47.

93. W. Bronger and L. Breil, Z. Anorg. Allg. Chem., 1998, 624, 1819.

94. B. Huang, K. Yvon, and P. Fischer, J. Alloys Comp., 1994, 210, 243.

95. B. Huang, P. Fischer, and K. Yvon, J. Alloys Comp., 1996, 245, L24.

96. A. P. Ginsberg and C. R. Sprinkle, Inorg. Synth., 1972, 13, 219 , and references therein.

97. N. T. Stetson, K. Yvon, and P. Fischer, Inorg. Chem., 1994, 33, 4598 .

98. E. Orgaz and M. Gupta, J. Alloys Comp., 1999, 293-295, 217.

99. M. Bortz, K. Yvon, and P. Fischer, J. Alloys Comp., 1994, 216, 39.

100. M. Bortz, A. Hewat, and K. Yvon, J. Alloys Comp., 1997, 248, L1.

101. M. Olofsson-Martensson, M. Kritikos, and D. Noréus, J. Am. Chem. Soc., 1999, 121, 10908.

102. H. Kohlmann, H. E. Fischer, and K. Yvon, Inorg. Chem., 2001, 40, 2608.

103. E. Orgaz, J. Alloys Comp., 2003, 356-357, 191.

104. M. Bortz, K. Yvon, and P. Fischer, J. Alloys Comp., 1994, 216, 43.

105. M. Bortz, A. Hewat, and K. Yvon, J. Alloys Comp., 1997, 253-254, 13 .

106. W. Bronger, S. Hasenberg, and G. Auffermann, Z. Anorg. Allg. Chem., 1996, 622, 1145.

107. W. Bronger, S. Hasenberg, and G. Auffermann, J. Alloys Comp., 1997, 257, 75.

108. M. Bortz, M. Gutmann, and K. Yvon, J. Alloys Comp., 1999, 285, L19.

109. K. Kadir and D. Noréus, J. Alloys Comp., 1994, 209, 213.

110. B. Huang, K. Yvon, and P. Fischer, J. Alloys Comp., 1995, 227, 116 .

111. N. T. Stetson and K. Yvon, J. Alloys Comp., 1995, 223, L4.

112. W. Bronger and L. à Brassard, Angew. Chem., Int. Ed. Engl., 1995, 34, 898.

113. B. Huang, F. Fauth, and K. Yvon, J. Alloys Comp., 1996, 244, L1.

114. W. Bronger and L. à Brassard, Z. Anorg. Allg. Chem., 1996, 622, 462 .

115. B. Huang, F. Gingl, F. Fauth, A. Hewat, and K. Yvon, J. Alloys Comp., 1997, 248, 13.

116. M. Olofsson, M. Kritikos, and D. Noréus, Inorg. Chem., 1998, 37, 2900 .

117. W. Bronger, G. Auffermann, and H. Schilder, Z. Anorg. Allg. Chem., 1998, 624, 497. 
118. W. Bronger and G. Auffermann, Z. Anorg. Allg. Chem., 1999, 625, 1147.

119. G. Auffermann, W. Bronger, R. M. Ibberson, and S. Hull, ISIS Facility Annual Report 2001-2002 - Highlights of ISIS Science, 2002, 22.

120. W. Bronger, T. Sommer, G. Auffermann, and P. Müller, J. Alloys Compd., 2002, 330-332, 536.

121. R. Cerny, J. M. Joubert, H. Kohlmann, and K. Yvon, J. Alloys Comp., 2002, 340, 180; see also R. Cerny, J. M. Joubert, and K. Yvon, Mater. Sci. Forum, 1998, 278-281, 121.

122. L. Guénée, V. Favre-Nicolin, and K. Yvon, J. Alloys Compd., 2003, 348, 129.

123. G. Renaudin, L. Guénée, and K. Yvon, J. Alloys Comp., 2003, 350, 145.

124. D. M. P. Mingos, and J. C. Hawes, Struct. Bonding (Berlin), 1985, 63, 1; see also R. Hoffmann, Angew. Chem., Int. Ed. Engl., 1982, 21, 711.
125. K. Kadir, and D. Noréus, Z. Phys. Chem., 1993, 179, 249; see also K. Kadir, P. Lundqvist, D. Noréus, and O. Rapp, Solid State Commun., 1993, 85, 891.

126. B. Nacken and W. Bronger, Z. Anorg. Allg. Chem., 1978, 439, 29.

127. J. D. Corbett, J. Solid State Chem., 1981, 39, 56.

128. K. Yvon, H. Kohlmann, and B. Bertheville, Chimia, 2001, 55, 505.

129. R. H. Crabtree, Acc. Chem. Res., 1990, 23, 95.

130. R. Griessen, Europhys. News, 2001, 32, 41.

131. R. Griessen and T. Riesterer, Top. Appl. Phys., 1988, 63, 219.

\section{Acknowledgment}

The work was supported by the Swiss National Science Foundation and the Swiss Federal Office of Energy. 